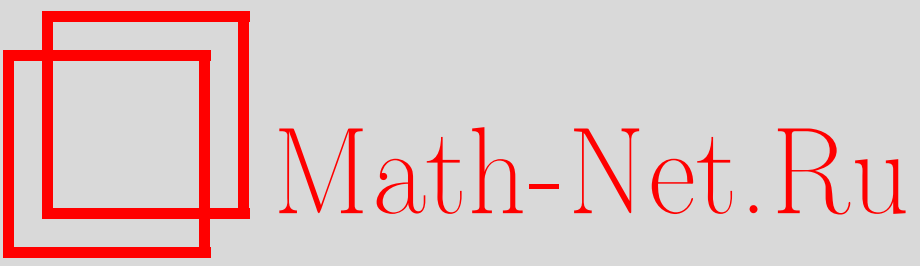

И. В. Тихонов, В. Б. Шерстюков, Д. Г. Цветкович, Обобщенные разложения Поповичу для полиномов Бернштейна от рационального модуля, Итоги науки и техн. Сер. Соврем. мат. и ее прил. Темат. обз., 2019, том 170, 71-117

DOI: https://doi.org/10.36535/0233-6723-2019-170-71-117

Использование Общероссийского математического портала Math-Net.Ru подразумевает, что вы прочитали и согласны с пользовательским соглашением

http://www.mathnet.ru/rus/agreement

Параметры загрузки:

IP : 54.237 .206 .68

26 апреля 2023 г., 13:45:25 


\title{
ОБОБЩЕННЫЕ РАЗЛОЖЕНИЯ ПОПОВИЧУ ДЛЯ ПОЛИНОМОВ БЕРНШТЕЙНА ОТ РАЦИОНАЛЬНОГО МОДУЛЯ
}

\author{
() 2019 г. . В. ТИХОНОВ, В. Б. ШЕРСТЮКОВ, Д. Г. ЦВЕТКОВИЧ
}

\begin{abstract}
АннотАция. В работе изучаются полиномы Бернштейна для простых негладких функций типа рационального модуля. Показано, что эти полиномы могут быть представлены в виде особых сумм регулярной структуры. По причинам исторического характера найденные выражения естественно называть «обобщенными разложениями Поповичу». Для записи обобщенных разложений разработан специальный формализм, связанный с комбинаторными вычислениями. На основе установленных формул дано полное описание характера сходимости изучаемых полиномов Бернштейна в комплексной плоскости. Обсуждается связь разложений Поповичу с вопросом распределения нулей полиномов Бернштейна. В заключительной части работы представлен ряд дополнительных новых соотношений для полиномов Бернштейна от рационального модуля.
\end{abstract}

Ключевые слова: полином Бернштейна, кусочно линейная функция, рациональный модуль, обобщенное разложение Поповичу, область сходимости, лемниската Канторовича, распределение нулей полинома.

\section{GENERALIZED POPOVICIU EXPANSIONS FOR BERNSTEIN POLYNOMIALS OF A RATIONAL MODULE}

\section{(c) 2019 I. V. TIKHONOV, V. B. SHERSTYUKOV, D. G. TSVETKOVICH}

Abstract. We study Bernstein polynomials for simple nonsmooth rational module functions. We show that these polynomials can be represented as special sums of regular structure. For historical reasons, the representations found are naturally called "generalized Popoviciu expansions." To write generalized expansions, we develop a special formalism based on combinatorial calculations. Based on the formulas obtained, we propose a complete description of the character of convergence of the Bernstein polynomials studied in the complex plane. We also discuss the relationship of generalized Popoviciu expansions with the distribution of zeros of Bernstein polynomials. In the final part of the paper, we present additional new relations for Bernstein polynomials of the rational module function.

Keywords and phrases: Bernstein polynomial, piecewise linear function, rational module, generalized Popoviciu expansion, domain of convergence, Kantorovich lemniscate, distribution of zeros of a polynomial.

AMS Subject Classification: 41A10, 41A25, 30C15, 30E10

Работа выполнена при поддержке Российского фонда фундаментальных исследований (проект № 18-01-00236). 
1. Введение. В 1935 г. молодой и впоследствии известный румынский математик Тиберий Поповичу отметил среди прочего в работе [53], что полиномы Бернштейна от функции $|x|$ на симметричном отрезке $[-1,1]$ допускают разложение в специальную сумму, рекуррентно изменяющуюся при увеличении номера полинома Бернштейна. Соответствующий результат был упомянут без доказательства в подстрочной сноске в конце работы (см. [53, с. 53-54]). Приведем перевод формулировки этого результата:

«Полином $P_{2 n}$ для функиии $|x|$ в интервале $(-1,1)$ равен

$$
\sum_{i=0}^{n}(-1)^{i}\left(\begin{array}{c}
1 / 2 \\
i
\end{array}\right)\left(1-x^{2}\right)^{i}
$$

т.е. в точности совпадает с п-й частичной суммой разложения Лебега

$$
|x|=\sum_{i=0}^{\infty}(-1)^{i}\left(\begin{array}{c}
1 / 2 \\
i
\end{array}\right)\left(1-x^{2}\right)^{i}
$$

Заметим еще, что в этом случае

$$
\frac{d^{2} P_{2 n}}{d x^{2}}=\frac{1 \cdot 3 \cdot 5 \cdots(2 n-1)}{2^{n-1}(n-1) !}(2 n-1)\left(1-x^{2}\right)^{n-1},
$$

что показывает наличие тесной связи между методами аппроксимачии Бернштейна, Ландау, и классическим методом Лебега».

Это неожиданное наблюдение долгое время оставалось незамеченным. На современном языке речь идет о полиномах Бернштейна

$$
P_{2 m}(f, x)=\frac{1}{2^{2 m}} \sum_{k=0}^{2 m} f\left(\frac{k}{m}-1\right) C_{2 m}^{k}(1+x)^{k}(1-x)^{2 m-k}, \quad m \in \mathbb{N},
$$

для стандартного модуля $f(x)=|x|$ на симметричном отрезке $[-1,1]$.

Утверждается, что при таком выборе $f(x)$ справедливо представление

$$
P_{2 m}(f, x)=1-\sum_{k=1}^{m} \frac{1}{2 k-1} 2^{-2 k} C_{2 k}^{k}\left(1-x^{2}\right)^{k}, \quad m \in \mathbb{N} .
$$

Данные полиномы совпадают с частичными суммами в известном разложении модуля, восходящем к Лебегу:

$$
\begin{aligned}
|x|=\sqrt{x^{2}}=\sqrt{1-\left(1-x^{2}\right)}=1-\frac{1}{2}\left(1-x^{2}\right)-\sum_{k=2}^{\infty} \frac{(2 k-3) ! !}{2^{k} k !}\left(1-x^{2}\right)^{k}= & \\
& =1-\sum_{k=1}^{\infty} \frac{1}{2 k-1} \frac{(2 k) !}{2^{2 k}(k !)^{2}}\left(1-x^{2}\right)^{k} .
\end{aligned}
$$

При этом также

$$
P_{2 m}^{\prime \prime}(f, x)=2 m \cdot 2^{-2 m} C_{2 m}^{m}\left(1-x^{2}\right)^{m-1}, \quad m \in \mathbb{N} .
$$

Можно показать, что полиномы $P_{2 m}^{\prime \prime}(f, x)$ образуют на $[-1,1]$ простую $\delta$-образную последовательность, использованную Ландау в его доказательстве теоремы Вейерштрасса (по поводу различных доказательств теоремы Вейерштрасса см. $[4,8,60])$.

Отметим также, что оригинальная формула второй производной в работе Поповичу дана с опечаткой. Правильный результат в его обозначениях должен выглядеть так:

$$
\frac{d^{2} P_{2 n}}{d x^{2}}=\frac{1 \cdot 3 \cdot 5 \cdots(2 n-1)}{2^{n-1}(n-1) !}\left(1-x^{2}\right)^{n-1} .
$$

Подробный вывод и обсуждение перечисленных формул в их современной трактовке см. в [24].

Использование симметричного отрезка не принципиально. В [19] упомянутые результаты Поповичу перенесены на случай функции $f(x)=|2 x-1|$, взятой на стандартном отрезке $[0,1]$ 
(см. также [22]). Установленные соотношения открывают новые возможности при оценке скорости сходимости полиномов Бернштейна к порождающей функции типа модуля (см. [21]) и при исследовании распределения нулей полиномов Бернштейна на плоскости $\mathbb{C}$ (см. $[25,26])$.

Для дальнейшего развития теории желательно иметь аналоги разложения Поповичу для полиномов Бернштейна

$$
B_{n}(f, z)=\sum_{k=0}^{n} f\left(\frac{k}{n}\right) C_{n}^{k} z^{k}(1-z)^{n-k}, \quad n \in \mathbb{N},
$$

комплексной переменной $z \in \mathbb{C}$, взятых от произвольного рационального модуля

$$
f(x)=|q x-p|, \quad x \in[0,1] .
$$

В настоящей работе мы даем полное решение поставленной задачи вместе с разбором некоторых частных случаев. Окончательные результаты весьма неожиданны и связаны с целым рядом нетривиальных аналитических и комбинаторных соображений.

2. Общие установки, соглашения, обозначения. Всюду далее работаем на стандартном отрезке $[0,1]$. Рассматриваем классические полиномы Бернштейна (1.1) с порождающими функциями вида (1.2) при соглашениях

$$
p, q \in \mathbb{N}, \quad q>p \geqslant 1, \quad \text { НОД }(p, q)=1 .
$$

Главной характеристикой функции (1.2) является ее точка излома

$$
x=\frac{p}{q} \in(0,1)
$$

или, чуть более длинно, абсиисса точки излома графика функиии $f(x)=|q x-p|$. Как станет ясно в дальнейшем, надо особо учитывать сочетание точки $(2.2)$ с точками из равномерных сеток

$$
0<\frac{1}{n}<\frac{2}{n}<\ldots<\frac{k}{n}<\ldots<\frac{n-1}{n}<\frac{n}{n}=1,
$$

используемых в определении полиномов Бернштейна (1.1).

Переменную $z \in \mathbb{C}$ в полиномах (1.1) сразу считаем комплексной, поскольку ряд естественных задач, связанных с полиномами Бернштейна, целесообразно изучать, выходя в комплексную плоскость (см. $[7,25])$. Основные сведения по теории полиномов Бернштейна изложены в монографиях $[3,36,49]$. В качестве дополнительных источников отметим $[4,8,11,37,38,52]$. Предварительная публикация части из наших результатов дана в [30,31].

Для биномиальных коэффициентов используем стандартный «русский» символ

$$
C_{n}^{k}=\frac{n !}{k !(n-k) !}=\left(\begin{array}{l}
n \\
k
\end{array}\right) .
$$

Через $\lfloor h\rfloor,\lceil h\rceil$ обозначаем пол и потолок числа $h \in \mathbb{R}$ (см. [5]), а через

$$
\{h\}=h-\lfloor h\rfloor, \quad\langle h\rangle=\lceil h\rceil-h
$$

- дробную и антидробную части числа $h \in \mathbb{R}$ соответственно. Понятие антидробной части не является общепринятым, но мы вводим значок $\langle h\rangle$, поскольку он сильно упрощает запись многих последующих формул. Справедливы очевидные тождества

$$
\lceil h\rceil-\lfloor h\rfloor=1, \quad\{h\}+\langle h\rangle=1, \quad h \in \mathbb{R} \backslash \mathbb{Z},
$$

при том, что $\lceil h\rceil-\lfloor h\rfloor=0$ и $\{h\}=\langle h\rangle=0$, если $h \in \mathbb{Z}$. 
3. Главный результат с краткими комментариями. Основная цель нашей работы состоит в доказательстве следующего утверждения.

Теорема 3.1. При всех значениях $m \in \mathbb{N} u r=0, \ldots, q-1$ для полиномов Бернштейна (1.1) от функции (1.2) при соглашениях (2.1) справедливо представление

$$
B_{q m+r}(f, z)=p+(q-2 p) z-\sum_{\nu=1}^{q-1} 2 z^{\lceil p \nu / q\rceil}(1-z)^{\nu-\lfloor p \nu / q\rfloor} \sum_{k=0}^{\varepsilon_{m}(\nu, r)} \frac{a_{p, q}(\nu)}{q k+\nu} C_{q k+\nu}^{p k+j_{p, q}(\nu)}\left(z^{p}(1-z)^{q-p}\right)^{k}
$$

¿əe

$$
\begin{gathered}
\varepsilon_{m}(\nu, r)= \begin{cases}m, & \nu \leqslant r-1, \\
m-1, & \nu \geqslant r\end{cases} \\
a_{p, q}(\nu)=\left\{\begin{array}{ll}
q\left\langle\frac{p \nu}{q}\right\rangle, & \nu \in \Delta_{p, q}^{(1)}, \\
q\left\{\frac{p \nu}{q}\right\}, & \nu \in \Delta_{p, q}^{(2)},
\end{array} \quad j_{p, q}(\nu)= \begin{cases}\left\lceil\frac{p \nu}{q}\right\rceil, & \nu \in \Delta_{p, q}^{(1)}, \\
\left\lfloor\frac{p \nu}{q}\right], & \nu \in \Delta_{p, q}^{(2)},\end{cases} \right. \\
\Delta_{p, q}^{(1)}=\left\{\nu \in \mathbb{N}: \quad \nu \leqslant q-1,\left\langle\frac{p \nu}{q}\right\rangle \leqslant \frac{p}{q}\right\}, \quad \Delta_{p, q}^{(2)}=\left\{\nu \in \mathbb{N}: \quad \nu \leqslant q-1,\left\langle\frac{p \nu}{q}\right\rangle>\frac{p}{q}\right\},
\end{gathered}
$$

причем величины (3.3) принимаят лишь целые значения из промежутков

$$
1 \leqslant a_{p, q}(\nu) \leqslant \max \{p, q-p-1\}, \quad 0 \leqslant j_{p, q}(\nu) \leqslant \min \{p, \nu\}, \quad \nu=1, \ldots, q-1 .
$$

Запись формулы (3.1) с обозначениями (3.2)-(3.4) допускает «косметические» изменения, связанные с различными техническими преобразованиями. Мы даем тот вариант, который, на наш взгляд, наиболее удобен на практике.

Видимая сложность и специфика обозначений связана с необходимостью учета всех возможных сочетаний параметров (2.1), определяющих расположение точки излома (2.2) среди точек равномерных сеток (2.3) при различных номерах $n \in \mathbb{N}$. Если значения $p, q$ взяты конкретно, то после проведения необходимых расчетов формула (3.1) упрощается, предоставляя достаточно наглядный результат - обобщенное разложение Поповичу для полиномов Бернштейна от функции (1.2).

Фиксированное значение $q \geqslant 2$ (знаменатель дроби $p / q$ ) задает постоянное число слагаемых во внешней сумме разложения (3.1) или, другими словами, задает постоянное количество внутренних сумм. Как видим, число этих сумм всегда будет $(q-1)$. Номер $\nu$ внутренней суммы в сочетании с выбранными $p, q$ определяет затем все числовые параметры.

Иначе говоря, формула (3.1) показывает, что всякий полином Бернштейна $B_{q m+r}(f, z)$ для функции (1.2) собирается «как конструктор» из следующих структурированных сумм:

$$
\mathfrak{S}_{p, q}^{d}(z ; \nu)=2 z^{\lceil p \nu / q\rceil}(1-z)^{\nu-\lfloor p \nu / q\rfloor} \sum_{k=0}^{d} \frac{a_{p, q}(\nu)}{q k+\nu} C_{q k+\nu}^{p k+j_{p, q}(\nu)}\left(z^{p}(1-z)^{q-p}\right)^{k} .
$$

Здесь $\nu=1, \ldots, q-1$ (порядковый номер суммы) и $d \in \mathbb{N} \cup\{0\}$ (верхний индекс суммы). При практическом использовании в конкретных примерах множители, стоящие перед знаком суммирования в формуле (3.6), бывает удобно вносить внутрь суммы.

Вся информация о выбираемом номере $n=q m+r$ полинома Бернштейна закладывается в способ индексации (3.2). Через каждые $q$ номеров ситуация циклически переходит на новый уровень. Внутри цикла все зависит от остатка $r$, определяющего число слагаемых в той или иной внутренней сумме (3.6) из общей формулы (3.1). При этом способ (3.2) учитывает важную особенность, а именно

$$
\varepsilon_{m}(\nu, 1)=\varepsilon_{m}(\nu, 0)=m-1, \quad \nu=1, \ldots, q-1 .
$$

Это согласуется со стандартным правилом склеивания

$$
B_{q m+1}(f, z)=B_{q m}(f, z), \quad m \in \mathbb{N},
$$


действующим для полиномов Бернштейна при выборе порождающей функции (1.2). Подробнее про правило склеивания см. обзор [22] и следующий раздел 4.

Используя суммы (3.6), можно переписать формулу (3.1) в зависимости от значения остатка $r$ следующим эквивалентным образом. При $r=0,1$ получаем

$$
B_{q m+1}(f, z)=B_{q m}(f, z)=p+(q-2 p) z-\sum_{\nu=1}^{q-1} \mathfrak{S}_{p, q}^{m-1}(z ; \nu)
$$

а при $r \geqslant 2$ (если такие остатки возможны) имеем

$$
B_{q m+r}(f, z)=p+(q-2 p) z-\left(\sum_{\nu=1}^{r-1} \mathfrak{S}_{p, q}^{m}(z ; \nu)+\sum_{\nu=r}^{q-1} \mathfrak{S}_{p, q}^{m-1}(z ; \nu)\right) .
$$

Отметим, что в простейшем случае - при $p=1$ и $q=2$-формула (3.9) будет содержать лишь одну сумму $\mathfrak{S}_{1,2}^{m-1}(z ; 1)$, а вторая формула (3.10) просто не нужна.

Пример 3.1. Пусть $f(x)=|2 x-1|, x \in[0,1]$. Прямой подсчет по формулам (3.1)-(3.4) для $p=1$ и $q=2$ дает результат

$$
B_{2 m+1}(f, z)=B_{2 m}(f, z)=1-2 z(1-z) \sum_{k=0}^{m-1} \frac{1}{2 k+1} C_{2 k+1}^{k+1}(z(1-z))^{k}, \quad m \in \mathbb{N} .
$$

Отсюда, с учетом тождества $2 C_{2 k+1}^{k+1}=C_{2 k+2}^{k+1}$, получаем более удобную запись

$$
B_{2 m+1}(f, z)=B_{2 m}(f, z)=1-\sum_{k=1}^{m} \frac{1}{2 k-1} C_{2 k}^{k}(z(1-z))^{k}, \quad m \in \mathbb{N} .
$$

Независимое доказательство (3.12) дано в [19] (см. также [22]). Сделаем еще один переход:

$$
B_{2 m+1}(f, z)=B_{2 m}(f, z)=1-\sum_{k=1}^{m} \frac{1}{2 k-1} 2^{-2 k} C_{2 k}^{k}(4 z(1-z))^{k}, \quad m \in \mathbb{N} .
$$

Это прямой аналог исходного разложения Поповичу (см. [53]) применительно к выбранному сейчас отрезку $[0,1]$. Форма (3.13) специально приспособлена для исследования сходимости данных полиномов Бернштейна: условие $4|z(1-z)| \leqslant 1$ дает точное описание комплексной области сходимости для $B_{n}(f, z)$ при выборе $f(x)=|2 x-1|$ (подробнее см. раздел 8).

Пример 3.2. Пусть $f(x)=|3 x-1|, x \in[0,1]$. Здесь $p=1, q=3$. Расчет по формулам (3.1)(3.4) после соответствующих преобразований дает представление

$$
\begin{aligned}
B_{3 m+1}(f, z)=B_{3 m}(f, z)=1+z-2 z & (1-z) \sum_{k=0}^{m-1} \frac{1}{3 k+1} C_{3 k+1}^{k}\left(z(1-z)^{2}\right)^{k}- \\
& -2 z(1-z)^{2} \sum_{k=0}^{m-1} \frac{2}{3 k+2} C_{3 k+2}^{k}\left(z(1-z)^{2}\right)^{k}, \quad m \in \mathbb{N},
\end{aligned}
$$

и затем

$$
\begin{aligned}
B_{3 m+2}(f, z)=1+z-2 z(1-z) \sum_{k=0}^{m} & \frac{1}{3 k+1} C_{3 k+1}^{k}\left(z(1-z)^{2}\right)^{k}- \\
& -2 z(1-z)^{2} \sum_{k=0}^{m-1} \frac{2}{3 k+2} C_{3 k+2}^{k}\left(z(1-z)^{2}\right)^{k}, \quad m \in \mathbb{N} .
\end{aligned}
$$

Внешне результаты похожи: единственное отличие (3.15) от (3.14) состоит в добавлении очередного $m$-го слагаемого в первую сумму. 
Подробные расчеты для представлений (3.11)-(3.15) и дальнейшие иллюстрации к общим формулам (3.1)-(3.4) будут даны в отдельном разделе 7 ниже.

Доказательство теоремы 3.1 проведем в три этапа. Сначала мы напомним специальную формулу Темпла, связывающую два последовательных полинома Бернштейна. Затем установим особую подготовительную формулу, представляющую полином $B_{q m+r}(f, z)$ через сумму попарных разностей предыдущих полиномов и, наконец, применим формулу Темпла к вычислению слагаемых в подготовительной формуле. Каждый этап составляет отдельный раздел нашей работы, причем заключительный, третий этап, является основным и самым трудным.

4. Формула Темпла. При сравнении двух последовательных полиномов Бернштейна $B_{n+1}(f, z)$ и $B_{n}(f, z)$, взятых от произвольной функции $f \in C[0,1]$, полезна формула

$$
B_{n+1}(f, z)-B_{n}(f, z)=\sum_{j=1}^{n} Q_{n, j}(f) z^{j}(1-z)^{n+1-j}, \quad n \in \mathbb{N},
$$

с коэффициентами

$$
Q_{n, j}(f)=C_{n+1}^{j} f\left(\frac{j}{n+1}\right)-C_{n}^{j} f\left(\frac{j}{n}\right)-C_{n}^{j-1} f\left(\frac{j-1}{n}\right) .
$$

Для коэффициентов $Q_{n, j}(f)$ возможны другие, эквивалентные записи. Например, используя стандартные обозначения для разделенных разностей первого и второго порядков

$$
\left[f ; x_{1}, x_{0}\right] \equiv \frac{f\left(x_{1}\right)-f\left(x_{0}\right)}{x_{1}-x_{0}}, \quad\left[f ; x_{2}, x_{1}, x_{0}\right] \equiv \frac{\left[f ; x_{2}, x_{1}\right]-\left[f ; x_{1}, x_{0}\right]}{x_{2}-x_{0}},
$$

выражение (4.2) можно преобразовать к виду

$$
Q_{n, j}(f)=-\frac{1}{n+1} C_{n-1}^{j-1}\left(\left[f ; \frac{j}{n}, \frac{j}{n+1}\right]-\left[f ; \frac{j}{n+1}, \frac{j-1}{n}\right]\right),
$$

а затем к виду

$$
Q_{n, j}(f)=-\frac{1}{n(n+1)} C_{n-1}^{j-1}\left[f ; \frac{j}{n}, \frac{j}{n+1}, \frac{j-1}{n}\right] .
$$

Записи (4.3) и (4.4) согласованы с последовательным расположением точек

$$
0 \leqslant \frac{j-1}{n}<\frac{j}{n+1}<\frac{j}{n} \leqslant 1
$$

действующим при всех $j=1,2, \ldots, n$.

Соотношение (4.1) с коэффициентами $Q_{n, j}(f)$, выраженными любым из перечисленных способов, будем называть формулой Темпла, поскольку впервые подобная формула была установлена в [59]. Независимо от [59] и чуть позже аналогичный результат отмечался в [34,57]. Простое доказательство формулы Темпла см. в [37, с. 115] или [38, с. 309-310] (в [38] использовано название «формула Авербах», ибо в одном из первоисточников [57] появление подобной формулы связывалось с именем американской студентки Бонни Авербах). Некоторые дополнительные подробности про формулу Темпла см. в [22, с. 141-143].

Изучаемые функции (1.2) при соглашениях (2.1) являются выпуклыми вниз на $[0,1]$. Соответственно, все вторые разности в формуле (4.4) будут заведомо неотрицательными, и коэффициенты $Q_{n, j}(f)$ оказываются неположительными. Формула (4.1) показывает, что тогда

$$
B_{n+1}(f, x) \leqslant B_{n}(f, x), \quad x \in[0,1],
$$

для любого $n \in \mathbb{N}$. Другими словами, в нашей ситуации полиномы Бернштейна сходятся к своей порождающей функции $f(x)=|q x-p|$ монотонно сверху на $[0,1]$. Это проявление общей теоремы Темпла-Арамэ-Авербах о монотонной сходимости полиномов Бернштейна к выпуклым порождающим функциям (см. $[34,57,59]$; см. также [37, с. 115] и [38, с. 309-310]).

При некоторых специальных номерах $n \in \mathbb{N}$ все точки (4.5) попадают на линейные участки рационального модуля $(1.2)$ с точкой излома $p / q \in(0,1)$. При таких $n$ все вторые разности в (4.4) будут равны нулю, коэффициенты $Q_{n, j}(f)$ обратятся в нуль, и соотношение (4.6) перейдет в равенство. Точный результат выглядит следующим образом. 
Лемма 4.1. Для полиномов Бернштейна (1.1), взятых от функиии $f(x)=|q x-p| n p u$ соглашениях (2.1), действует правило склеивания $B_{q m+1}(f, z)=B_{q m}(f, z)$ при всех $m \in \mathbb{N}$.

Доказательство. При номерах $n=q m$ в коэффициентах (4.4) фигурируют упорядоченные тройки точек

$$
\frac{j-1}{q m}, \quad \frac{j}{q m+1}, \quad \frac{j}{q m}, \quad j=1,2, \ldots, q m .
$$

Заметим, что

$$
\begin{aligned}
& 0 \leqslant \frac{j-1}{q m}<\frac{j}{q m+1}<\frac{j}{q m} \leqslant \frac{p}{q}, \quad j \leqslant p m, \\
& \frac{p}{q} \leqslant \frac{j-1}{q m}<\frac{j}{q m+1}<\frac{j}{q m} \leqslant 1, \quad j \geqslant p m+1 .
\end{aligned}
$$

Таким образом, любая тройка точек (4.7) укладывается на соответствующий линейный участок функции $f(x)=|q x-p|$, слева или справа от точки излома $x=p / q$. Согласно (4.4) в формуле Темпла для разности $B_{q m+1}(f, z)-B_{q m}(f, z)$ все коэффициенты $Q_{q m, j}(f)$ будут равны нулю. Следовательно, выполняется заявленное правило склеивания.

В связи с правилом склеивания отметим работы [47,50,51] и обзор [22], где обсуждается более общий случай кусочно линейных порождающих функций с несколькими рациональными точками излома. В [23] показано, что для полиномов Бернштейна на симметричном отрезке $[-1,1]$ формулировка правила склеивания требует определенной коррекции. Эти результаты, впрочем, сейчас не понадобятся.

По-прежнему работаем с функцией $f(x)=|q x-p|$, заданной на $[0,1]$ при соглашениях $(2.1)$. Покажем, как можно выразить полином $B_{q m+r}(f, z)$ через последовательные разности предыдущих полиномов Бернштейна при учете правила склеивания из леммы 4.1.

5. Подготовительная формула для записи полиномов Бернштейна. Удобно сразу установить следующий общий принцип.

Лемма 5.1. Пусть $\left\{A_{n}\right\}_{n=1}^{\infty}-$ последовательность однотипных элементов (чисел или функиий), в которой выполняется правило склеивания

$$
A_{q m+1}=A_{q m}, \quad m \in \mathbb{N},
$$

с некоторым $q \in \mathbb{N}, q \geqslant 2$. Тогда справедливо представление

$$
A_{q m+r}=A_{1}+\sum_{\nu=1}^{q-1} \sum_{k=0}^{\varepsilon_{m}(\nu, r)}\left(A_{q k+\nu+1}-A_{q k+\nu}\right), \quad m \in \mathbb{N}, \quad r=0,1, \ldots, q-1,
$$

где верхний индекс суммирования $\varepsilon_{m}(\nu, r)$ определяется формулой (3.2).

Доказательство. Используем элементарный прием. Запишем

$$
A_{n}=A_{1}+\left(A_{2}-A_{1}\right)+\left(A_{3}-A_{2}\right)+\ldots+\left(A_{n-1}-A_{n-2}\right)+\left(A_{n}-A_{n-1}\right) .
$$

Рассмотрим сначала номера $n=q m$ и $n=q m+1$ с некоторым $m \in \mathbb{N}$, когда по условию (5.1) элементы $A_{q m}$ и $A_{q m+1}$ совпадают. Но при $n=q m+1$ в записи (5.3) оказывается ровно $q m$ скобок. Группируя их по $q$ скобок подряд, получаем выражение

$$
A_{q m}=A_{q m+1}=A_{1}+\sum_{k=0}^{m-1} \sum_{\nu=1}^{q}\left(A_{q k+\nu+1}-A_{q k+\nu}\right), \quad m \in \mathbb{N} .
$$

На основании все того же правила (5.1) при $\nu=q$ имеем

$$
\left.\left(A_{q k+\nu+1}-A_{q k+\nu}\right)\right|_{\nu=q}=A_{q(k+1)+1}-A_{q(k+1)}=0, \quad k=0, \ldots, m-1 .
$$


Соответственно,

$$
A_{q m}=A_{q m+1}=A_{1}+\sum_{k=0}^{m-1} \sum_{\nu=1}^{q-1}\left(A_{q k+\nu+1}-A_{q k+\nu}\right)=A_{1}+\sum_{\nu=1}^{q-1} \sum_{k=0}^{m-1}\left(A_{q k+\nu+1}-A_{q k+\nu}\right)
$$

при всех $m \in \mathbb{N}$.

Для элемента $A_{q m+r}$ при $r \in\{2, \ldots, q-1\}$ (если такие остатки $r$ возможны) к формуле (5.4) добавляется $(r-1)$ слагаемых в том смысле, что

$$
A_{q m+r}=A_{q m+1}+\left(A_{q m+2}-A_{q m+1}\right)+\ldots+\left(A_{q m+r}-A_{q m+r-1}\right) .
$$

Отсюда с учетом (5.4) получаем

$$
A_{q m+r}=A_{1}+\sum_{\nu=1}^{r-1} \sum_{k=0}^{m}\left(A_{q k+\nu+1}-A_{q k+\nu}\right)+\sum_{\nu=r}^{q-1} \sum_{k=0}^{m-1}\left(A_{q k+\nu+1}-A_{q k+\nu}\right)
$$

при всех $m \in \mathbb{N}$ и $r=2, \ldots, q-1$.

Введем символ $\varepsilon_{m}(\nu, r)$ по следующему правилу:

(а) если $r=0,1$, то

$$
\varepsilon_{m}(\nu, r)=m-1, \quad \nu=1, \ldots, q-1
$$

(b) если $r \geqslant 2$, то

$$
\varepsilon_{m}(\nu, r)= \begin{cases}m, & \nu=1, \ldots, r-1 \\ m-1, & \nu=r, \ldots, q-1\end{cases}
$$

Тогда формулы (5.4), (5.5) получают общую компактную запись (5.2), пригодную при всех значениях $m \in \mathbb{N}$ и $r=0,1, \ldots, q-1$. Осталось заметить, что правила (5.6), (5.7), определяющие символ $\varepsilon_{m}(\nu, r)$, коротко выражаются в виде (3.2). Лемма доказана.

Выберем теперь в качестве элементов $A_{n}$ полиномы Бернштейна $B_{n}(f, z)$ от функции $(1.2)$ при соглашениях (2.1). Для указанных полиномов по лемме 4.1 выполняется правило склеивания (3.8); следовательно, все условия леммы 5.1 выполнены. На основании формулы (5.2) получаем представление

$$
B_{q m+r}(f, z)=B_{1}(f, z)+\sum_{\nu=1}^{q-1} \sum_{k=0}^{\varepsilon_{m}(\nu, r)}\left(B_{q k+\nu+1}(f, z)-B_{q k+\nu}(f, z)\right)
$$

при всех $m \in \mathbb{N}$ и $r=0,1, \ldots, q-1$ с символом $\varepsilon_{m}(\nu, r)$, заданным по правилу (3.2).

Назовем (5.8) подготовительной формулой для полинома $B_{q m+r}(f, z)$. Используя эту формулу, установим основное разложение (3.1) с величинами (3.2)-(3.4). Для этого надо вычислить все разности, входящие в сумму (5.8).

6. Вычисление разностей и доказательство главного результата. Для порождающей функции $f(x)=|q x-p|$, заданной на $[0,1]$ при соглашениях $(2.1)$, рассмотрим конкретный полином Бернштейна $B_{q m+r}(f, z)$ в записи (5.8). Покажем, что разности

$$
B_{q k+\nu+1}(f, z)-B_{q k+\nu}(f, z),
$$

образующие сумму (5.8), допускают конструктивное вычисление.

Лемма 6.1. При всех $k \in \mathbb{N} \cup\{0\} u \nu=1, \ldots, q-1$ справедлива формула

$$
B_{q k+\nu+1}(f, z)-B_{q k+\nu}(f, z)=-\frac{2 a_{p, q}(\nu)}{q k+\nu} C_{q k+\nu}^{p k+j_{p, q}(\nu)} z^{p k+\lceil p \nu / q\rceil}(1-z)^{(q-p) k+\nu-\lfloor p \nu / q\rfloor},
$$

где величины $a_{p, q}(\nu), j_{p, q}(\nu)$ определены формулами (3.3) и принимают лишь целье значения из промежутков (3.5). 
Доказательство. По формуле Темпла (4.1) разности (6.1) представимы в виде

$$
B_{q k+\nu+1}(f, z)-B_{q k+\nu}(f, z)=\sum_{j=1}^{q k+\nu} Q_{q k+\nu, j}(f) z^{j}(1-z)^{q k+\nu+1-j}
$$

где, согласно (4.4), имеем

$$
Q_{q k+\nu, j}(f)=-\frac{1}{(q k+\nu)(q k+\nu+1)} C_{q k+\nu-1}^{j-1}\left[f ; \frac{j}{q k+\nu}, \frac{j}{q k+\nu+1}, \frac{j-1}{q k+\nu}\right] .
$$

Напомним (см. (4.5)), что запись (6.4) учитывает последовательное расположение на $[0,1]$ точек

$$
\frac{j-1}{q k+\nu}<\frac{j}{q k+\nu+1}<\frac{j}{q k+\nu}, \quad j=1, \ldots, q k+\nu .
$$

Если какая-либо тройка точек из (6.5) попадает на линейный участок функции $f(x)=|q x-p|$, слева или справа от точки излома $p / q \in(0,1)$, то вторая разность в (6.4) будет равна нулю и коэффициент $Q_{q k+\nu, j}(f)$ обратится в нуль. Для того чтобы коэффициент $Q_{q k+\nu, j}(f)$ был отличен от нуля при некотором $j \in\{1, \ldots, q k+\nu\}$, необходимо и достаточно, чтобы выполнялось соотношение

$$
\frac{j-1}{q k+\nu}<\frac{p}{q}<\frac{j}{q k+\nu}
$$

т.е. точка излома $x=p / q$ должна оказаться между крайними точками разделенной разности в (6.4). Разрешая элементарное неравенство (6.6), получаем, что

$$
p k+\frac{p \nu}{q}<j<p k+\frac{p \nu}{q}+1 .
$$

Учитывая несократимость дроби $p / q$ и тот факт, что $\nu \in\{1, \ldots, q-1\}$, замечаем, что число $p \nu / q$ будет заведомо нецелым, и, значит, оценке (6.7) удовлетворяет единственное целое значение

$$
j_{0} \equiv p k+\left\lceil\frac{p \nu}{q}\right\rceil .
$$

Ясно, что $1 \leqslant j_{0} \leqslant q k+\nu$, т.е. $j_{0}$ попадает в нужный диапазон значений, указанный в (6.5).

Итак, при условиях $k \in \mathbb{N} \cup\{0\}$ и $\nu \in\{1, \ldots, q-1\}$ в формуле (6.3) имеется ровно одно слагаемое, отличное от тождественного нуля - то, которое отвечает номеру $j=j_{0}$ вида (6.8). Взяв такой номер, вычислим соответствующий коэффициент (6.4).

Основную часть составляет вычисление разделенной разности

$$
\left[f ; \frac{j_{0}}{q k+\nu}, \frac{j_{0}}{q k+\nu+1}, \frac{j_{0}-1}{q k+\nu}\right], \quad j_{0} \equiv p k+\left\lceil\frac{p \nu}{q}\right\rceil,
$$

точки которой коротко обозначим

$$
x_{0} \equiv \frac{j_{0}-1}{q k+\nu}, \quad x_{1} \equiv \frac{j_{0}}{q k+\nu+1}, \quad x_{2} \equiv \frac{j_{0}}{q k+\nu} .
$$

Выбор номера $j_{0}$ в соответствии с (6.6) дает соотношение $x_{0}<p / q<x_{2}$. Дальнейшие рассуждения зависят от расположения точки $x_{1}$ - находится ли она слева или справа от $p / q$. Чётко разделим эти два случая.

Случай 1. Пусть $x_{1} \leqslant p / q$ (допуская здесь нестрогое неравенство). В подробной записи

$$
\frac{j_{0}}{q k+\nu+1} \leqslant \frac{p}{q}
$$

Подставляя в (6.11) значение $j_{0}$ из формулы (6.8), получаем, что

$$
\left\lceil\frac{p \nu}{q}\right\rceil \leqslant \frac{p \nu}{q}+\frac{p}{q} \sim\left\lceil\frac{p \nu}{q}\right\rceil-\frac{p \nu}{q} \leqslant \frac{p}{q} .
$$


Выразим результат через антидробную часть из формулы (2.4) в виде

$$
\left\langle\frac{p \nu}{q}\right\rangle \leqslant \frac{p}{q}
$$

Соотношение (6.12) рассмотрим как условие на $\nu$, определяющее множество

$$
\Delta_{p, q}^{(1)}=\left\{\nu \in \mathbb{N}: \nu \leqslant q-1,\left\langle\frac{p \nu}{q}\right\rangle \leqslant \frac{p}{q}\right\} .
$$

Это первое из множеств (3.4). Итак, при всех $\nu$ из (6.13) получаем конфигурацию точек

$$
x_{0}<x_{1} \leqslant \frac{p}{q}<x_{2}
$$

со значениями $x_{0}, x_{1}, x_{2}$ из формулы (6.10).

Приступим к вычислению разделенной разности (6.9). По определению

$$
\left[f ; x_{2}, x_{1}, x_{0}\right] \equiv \frac{1}{x_{2}-x_{0}}\left(\left[f ; x_{2}, x_{1}\right]-\left[f ; x_{1}, x_{0}\right]\right) .
$$

Учитывая конфигурацию (6.14) и структуру функции $f(x)=|q x-p|$, находим значения

$$
\left[f ; x_{2}, x_{1}\right] \equiv \frac{f\left(x_{2}\right)-f\left(x_{1}\right)}{x_{2}-x_{1}}=\frac{q\left(x_{1}+x_{2}\right)-2 p}{x_{2}-x_{1}}, \quad\left[f ; x_{1}, x_{0}\right] \equiv \frac{f\left(x_{1}\right)-f\left(x_{0}\right)}{x_{1}-x_{0}}=-q .
$$

Подстановка (6.16) в (6.15) дает результат

$$
\left[f ; x_{2}, x_{1}, x_{0}\right]=\frac{2\left(q x_{2}-p\right)}{\left(x_{2}-x_{0}\right)\left(x_{2}-x_{1}\right)} .
$$

Воспользуемся явными выражениями (6.10), откуда

$$
q x_{2}-p=\frac{q j_{0}-p q k-p \nu}{q k+\nu}, \quad x_{2}-x_{0}=\frac{1}{q k+\nu}, \quad x_{2}-x_{1}=\frac{j_{0}}{(q k+\nu)(q k+\nu+1)} .
$$

Заключительная подстановка (6.18) в (6.17) приводит к формуле

$$
\left[f ; x_{2}, x_{1}, x_{0}\right]=\frac{2}{j_{0}}(q k+\nu)(q k+\nu+1)\left(q j_{0}-p q k-p \nu\right) .
$$

Последняя скобка с учетом явного выражения (6.8) равна

$$
q j_{0}-p q k-p \nu=q\left\lceil\frac{p \nu}{q}\right\rceil-p \nu=q\left(\left\lceil\frac{p \nu}{q}\right\rceil-\frac{p \nu}{q}\right)=q\left\langle\frac{p \nu}{q}\right\rangle .
$$

Здесь вновь использована антидробная часть из формулы (2.4).

Подведем итог. Для точек (6.10), находящихся в конфигурации (6.14), соответствующая вторая разность (6.9) может быть записана в виде

$$
\left[f ; \frac{j_{0}}{q k+\nu}, \frac{j_{0}}{q k+\nu+1}, \frac{j_{0}-1}{q k+\nu}\right]=\frac{2}{j_{0}}(q k+\nu)(q k+\nu+1) \cdot q\left\langle\frac{p \nu}{q}\right\rangle .
$$

Поскольку значение $j_{0}$ из формулы (6.8) отвечает единственному ненулевому коэффициенту (6.4), то при вычислении такого коэффициента с учетом (6.19) получим

$$
Q_{q k+\nu, j_{0}}(f)=-\frac{2}{j_{0}} C_{q k+\nu-1}^{j_{0}-1} \cdot q\left\langle\frac{p \nu}{q}\right\rangle .
$$

Используем в (6.20) элементарное тождество

$$
\frac{1}{m} C_{n-1}^{m-1}=\frac{1}{n} C_{n}^{m}, \quad n, m \in \mathbb{N}, \quad n \geqslant m
$$


Отсюда

$$
\begin{aligned}
Q_{q k+\nu, j_{0}}(f)=-\frac{2}{q k+\nu} C_{q k+\nu}^{j_{0}} \cdot q\left\langle\frac{p \nu}{q}\right\rangle=-\frac{2 q}{q k+\nu}\left\langle\frac{p \nu}{q}\right\rangle C_{q k+\nu}^{p k+\lceil p \nu / q\rceil} & = \\
& =-\frac{2 a_{p, q}(\nu)}{q k+\nu} C_{q k+\nu}^{p k+j_{p, q}(\nu)} .
\end{aligned}
$$

Здесь величины $a_{p, q}(\nu), j_{p, q}(\nu)$, принимающие лишь натуральные значения, определены правилом

$$
a_{p, q}(\nu)=q\left\langle\frac{p \nu}{q}\right\rangle, \quad j_{p, q}(\nu)=\left\lceil\frac{p \nu}{q}\right\rceil, \quad \nu \in \Delta_{p, q}^{(1)} .
$$

Формулы (6.22) суть первая составная часть общих выражений (3.3).

Подставим теперь единственный ненулевой коэффициент (6.21) в формулу Темпла (6.3). Учитывая вид (6.8) для $j=j_{0}$, получим в итоге

$$
\begin{gathered}
B_{q k+\nu+1}(f, z)-B_{q k+\nu}(f, z)=-\frac{2 a_{p, q}(\nu)}{q k+\nu} C_{q k+\nu}^{p k+j_{p, q}(\nu)} z^{j_{0}}(1-z)^{q k+\nu+1-j_{0}}= \\
=-\frac{2 a_{p, q}(\nu)}{q k+\nu} C_{q k+\nu}^{p k+j_{p, q}(\nu)} z^{p k+\lceil p \nu / q\rceil}(1-z)^{(q-p) k+\nu-}(\lceil p \nu / q\rceil-1)= \\
=-\frac{2 a_{p, q}(\nu)}{q k+\nu} C_{q k+\nu}^{p k+j_{p, q}(\nu)} z^{p k+\lceil p \nu / q\rceil}(1-z)^{(q-p) k+\nu-\lfloor p \nu / q\rfloor} .
\end{gathered}
$$

В последнем переходе использовали элементарное тождество $\lceil p \nu / q\rceil-1=\lfloor p \nu / q\rfloor$, заведомо верное, ибо $p \nu / q \notin \mathbb{Z}$. В итоге получили нужное выражение (6.2). Но оно доказано пока лишь для значений $\nu$ из множества (6.13), когда точки (6.10) находятся в конфигурации (6.14).

Разберем теперь вторую возможность.

Случай 2. Пусть точки (6.10) находятся в конфигурации

$$
x_{0}<\frac{p}{q}<x_{1}<x_{2}
$$

со строгим неравенством $x_{1}>p / q$. По аналогии с (6.11) подробная запись

$$
x_{1} \equiv \frac{j_{0}}{q k+\nu+1}>\frac{p}{q}
$$

при подстановке значения $j_{0}$ из формулы (6.8) дает соотношение

$$
\left\langle\frac{p \nu}{q}\right\rangle>\frac{p}{q}
$$

двойственное к прежнему (6.12).

Соответственно рассмотрим множество

$$
\Delta_{p, q}^{(2)}=\left\{\nu \in \mathbb{N}: \nu \leqslant q-1,\left\langle\frac{p \nu}{q}\right\rangle>\frac{p}{q}\right\}
$$

дополнительное к множеству $\Delta_{p, q}^{(1)}$ из формулы (6.13). При всех $\nu$ из (6.25) имеем конфигурацию точек (6.24) со значениями $x_{0}, x_{1}, x_{2}$ из формулы (6.10).

Приступим к вычислению разделенной разности (6.9). Вновь пользуемся формулой (6.15), для которой, учитывая конфигурацию (6.24) и структуру функции $f(x)=|q x-p|$, находим значения

$$
\left[f ; x_{2}, x_{1}\right] \equiv \frac{f\left(x_{2}\right)-f\left(x_{1}\right)}{x_{2}-x_{1}}=q, \quad\left[f ; x_{1}, x_{0}\right] \equiv \frac{f\left(x_{1}\right)-f\left(x_{0}\right)}{x_{1}-x_{0}}=\frac{q\left(x_{0}+x_{1}\right)-2 p}{x_{1}-x_{0}} .
$$

Подстановка (6.26) в (6.15) дает результат

$$
\left[f ; x_{2}, x_{1}, x_{0}\right]=\frac{2\left(p-q x_{0}\right)}{\left(x_{2}-x_{0}\right)\left(x_{1}-x_{0}\right)} .
$$


Учитывая явные выражения (6.10), получаем

$$
p-q x_{0}=\frac{p q k+p \nu-q j_{0}+q}{q k+\nu}, \quad x_{2}-x_{0}=\frac{1}{q k+\nu}, \quad x_{1}-x_{0}=\frac{q k+\nu-j_{0}+1}{(q k+\nu)(q k+\nu+1)} .
$$

Заключительная подстановка (6.28) в (6.27) приводит к формуле

$$
\left[f ; x_{2}, x_{1}, x_{0}\right]=\frac{2}{q k+\nu-j_{0}+1}(q k+\nu)(q k+\nu+1)\left(p q k+p \nu-q j_{0}+q\right) .
$$

Последняя скобка с учетом явного выражения (6.8) равна

$$
p q k+p \nu-q j_{0}+q=p \nu-q\left\lceil\frac{p \nu}{q}\right\rceil+q=q\left(\frac{p \nu}{q}-\left\lceil\frac{p \nu}{q}\right\rceil+1\right)=q\left(\frac{p \nu}{q}-\left\lfloor\frac{p \nu}{q}\right\rfloor\right)=q\left\{\frac{p \nu}{q}\right\} .
$$

Здесь применялось первое из тождеств $(2.5)$ с учетом того, что $p \nu / q \notin \mathbb{Z}$.

Итак, для точек (6.10), находящихся в конфигурации (6.24), соответствующая вторая разность (6.9) представима в виде

$$
\left[f ; \frac{j_{0}}{q k+\nu}, \frac{j_{0}}{q k+\nu+1}, \frac{j_{0}-1}{q k+\nu}\right]=\frac{2}{q k+\nu-j_{0}+1}(q k+\nu)(q k+\nu+1) \cdot q\left\{\frac{p \nu}{q}\right\} .
$$

Поскольку значение $j_{0}$ из формулы (6.8) отвечает единственному ненулевому коэффициенту (6.4), то при вычислении такого коэффициента с учетом (6.29) получим

$$
Q_{q k+\nu, j_{0}}(f)=-\frac{2}{q k+\nu-j_{0}+1} C_{q k+\nu-1}^{j_{0}-1} \cdot q\left\{\frac{p \nu}{q}\right\} .
$$

Применим в (6.30) элементарное тождество

$$
\frac{1}{n-m+1} C_{n-1}^{m-1}=\frac{1}{n} C_{n}^{m-1}, \quad n, m \in \mathbb{N}, \quad n \geqslant m .
$$

В результате

$$
\begin{aligned}
Q_{q k+\nu, j_{0}}(f)=-\frac{2}{q k+\nu} C_{q k+\nu}^{j_{0}-1} \cdot q\left\{\frac{p \nu}{q}\right\} & =-\frac{2 q}{q k+\nu}\left\{\frac{p \nu}{q}\right\} C_{q k+\nu}^{p k+\lceil p \nu / q\rceil-1}= \\
& =-\frac{2 q}{q k+\nu}\left\{\frac{p \nu}{q}\right\} C_{q k+\nu}^{p k+\lfloor p \nu / q\rfloor}=-\frac{2 a_{p, q}(\nu)}{q k+\nu} C_{q k+\nu}^{p k+j_{p, q}(\nu)} .
\end{aligned}
$$

Здесь величины $a_{p, q}(\nu), j_{p, q}(\nu)$ определены по правилу

$$
a_{p, q}(\nu)=q\left\{\frac{p \nu}{q}\right\}, \quad j_{p, q}(\nu)=\left\lfloor\frac{p \nu}{q}\right\rfloor, \quad \nu \in \Delta_{p, q}^{(2)} .
$$

Формулы (6.32) являются второй составной частью общих выражений (3.3).

С учетом введенных обозначений (6.32) подставим единственный ненулевой коэффициент (6.31) в формулу Темпла (6.3). Поскольку формальная окончательная запись (6.31) совпадает с прежним видом (6.21), то соответствующая выкладка в точности повторяет цепочку (6.23). В итоге нужное соотношение (6.2) доказано при всех значениях

$$
\nu \in\{1, \ldots, q-1\}=\Delta_{p, q}^{(1)} \cup \Delta_{p, q}^{(2)}, \quad \Delta_{p, q}^{(1)} \cap \Delta_{p, q}^{(2)}=\varnothing,
$$

и, разумеется, при всех значениях $k \in \mathbb{N} \cup\{0\}$.

Осталось обсудить величины $a_{p, q}(\nu), j_{p, q}(\nu)$. То, что их значения всегда будут целыми и попадут в промежутки (3.5), видно из выражений (3.3). В проверке нуждается лишь верхняя граница для $a_{p, q}(\nu)$. Действительно, пусть $\nu \in \Delta_{p, q}^{(1)}$. Тогда $\langle p \nu / q\rangle \leqslant p / q$, и из (3.3) следует, что

$$
a_{p, q}(\nu)=q\left\langle\frac{p \nu}{q}\right\rangle \leqslant q \cdot \frac{p}{q}=p .
$$

Если же $\nu \in \Delta_{p, q}^{(2)}$, то $\langle p \nu / q\rangle>p / q$, и из (3.3) следует, что

$$
a_{p, q}(\nu)=q\left\{\frac{p \nu}{q}\right\}=q\left(1-\left\langle\frac{p \nu}{q}\right\rangle\right)<q\left(1-\frac{p}{q}\right)=q-p .
$$


Тем самым, заведомо $a_{p, q}(\nu) \leqslant \max \{p, q-p-1\}$, что и утверждалось в (3.5). Лемма доказана.

Для доказательства главного результата - теоремы 3.1 - все готово. Воспользуемся подготовительной формулой (5.8). Подставив туда найденные выражения (6.2) для разностей (6.1), получим

$$
\begin{aligned}
B_{q m+r}(f, z) & =B_{1}(f, z)+\sum_{\nu=1}^{q-1} \sum_{k=0}^{\varepsilon_{m}(\nu, r)}\left(B_{q k+\nu+1}(f, z)-B_{q k+\nu}(f, z)\right)= \\
& =B_{1}(f, z)+\sum_{\nu=1}^{q-1} \sum_{k=0}^{\varepsilon_{m}(\nu, r)}\left(-\frac{2 a_{p, q}(\nu)}{q k+\nu} C_{q k+\nu}^{p k+j_{p, q}(\nu)} z^{p k+\lceil p \nu / q\rceil}(1-z)^{(q-p) k+\nu-\lfloor p \nu / q\rfloor}\right)
\end{aligned}
$$

при всех $m \in \mathbb{N}$ и $r=0,1, \ldots, q-1$ с символом $\varepsilon_{m}(\nu, r)$, заданным по правилу (3.2). Здесь величины $a_{p, q}(\nu), j_{p, q}(\nu)$ определены формулой (3.3) и принимают лишь целые значения из промежутков (3.5). Для функции $f(x)=|q x-p|$, взятой на $[0,1]$, первый полином Бернштейна (1.1) имеет вид $B_{1}(f, z)=p+(q-2 p) z$. Учитывая это и проводя простейшие преобразования в (6.33), получаем окончательную запись (3.1). Теорема 3.1 полностью доказана.

7. Примеры нахождения конкретных разложений. Покажем на примерах, как работает формула (3.1). Последовательность наших действий достаточно проста. При заданной функции (1.2), т.е. при заданных значениях $p, q$, соответствующих ограничениям (2.1), вводим множество

$$
\Delta \equiv\{\nu \in \mathbb{N}: \nu \leqslant q-1\}=\{1, \ldots, q-1\}
$$

и производим отбор номеров

$$
\nu \in \Delta:\left\langle\frac{p \nu}{q}\right\rangle \leqslant \frac{p}{q}
$$

Здесь $\langle\cdot\rangle$ - знак антидробной части из формулы (2.4). Согласно (3.4) условие (7.2) определяет множество $\Delta_{p, q}^{(1)}$. Соответственно $\Delta_{p, q}^{(2)}=\Delta \backslash \Delta_{p, q}^{(1)}$. Затем, в зависимости от попадания номера $\nu$ в множество $\Delta_{p, q}^{(1)}$ или множество $\Delta_{p, q}^{(2)}$, происходит вычисление величин $a_{p, q}(\nu), j_{p, q}(\nu)$ по правилу (3.3). Найденные значения подставляются в формулу (3.1) и выполняются простейшие преобразования по окончательной «доводке» ответа.

Для представления полученного результата удобно использовать также структурированные суммы (3.6) с записями (3.9), (3.10), эквивалентными общей формуле (3.1). Кроме того, основная формула (6.2) позволяет указать рекуррентные соотношения, действующие для полиномов

$$
B_{q m}(f, z)=B_{q m+1}(f, z), \quad B_{q m+2}(f, z), \quad \ldots, \quad B_{q m+q-1}(f, z), \quad B_{q(m+1)}(f, z) .
$$

Серия (7.3) образует один полный цикл.

Пример 7.1. Пусть $f(x)=|2 x-1|$ на $[0,1]$, как в примере 3.1. Здесь $p=1, q=2$ с множеством (7.1), состоящим из одного номера $\nu=1$. Условие (7.2) дает выражение $\langle\nu / 2\rangle \leqslant 1 / 2$, очевидно выполненное при $\nu=1$; соответственно $\Delta_{1,2}^{(1)}=\{1\}$ и $\Delta_{1,2}^{(2)}=\varnothing$. Проводя подсчет величин (3.3), получаем

$$
\nu=1 \in \Delta_{1,2}^{(1)} \quad \Longrightarrow \quad a_{1,2}(1)=2\left\langle\frac{1}{2}\right\rangle=1, \quad j_{1,2}(1)=\left\lceil\frac{1}{2}\right\rceil=1 .
$$

Последующая подстановка в формулу (3.6) дает единственную структурированную сумму

$$
\mathfrak{S}_{1,2}^{d}(z ; 1)=2 z^{\lceil 1 / 2\rceil}(1-z)^{1-\lfloor 1 / 2\rfloor} \sum_{k=0}^{d} \frac{1}{2 k+1} C_{2 k+1}^{k+1}(z(1-z))^{k}, \quad d \in \mathbb{N} \cup\{0\} .
$$

Отсюда, с учетом формулы (3.9), требующей сейчас лишь одной суммы $\mathfrak{S}_{1,2}^{m-1}(z ; 1)$, получаем нужное выражение (3.11), а затем и окончательные выражения (3.12), (3.13). 
Пример 7.2. Пусть $f(x)=|3 x-1|$ на $[0,1]$, как в примере 3.2 . Здесь $p=1, q=3$. Множество (7.1) состоит из двух номеров $\nu=1$ и $\nu=2$. Условие (7.2) дает выражение $\langle\nu / 3\rangle \leqslant 1 / 3$, выполненное при $\nu=2$; соответственно $\Delta_{1,3}^{(1)}=\{2\}$ и $\Delta_{1,3}^{(2)}=\{1\}$. Подсчет величин (3.3) приводит к следующим результатам:

$$
\begin{aligned}
& \nu=1 \in \Delta_{1,3}^{(2)} \quad \Longrightarrow \quad a_{1,3}(1)=3\left\{\frac{1}{3}\right\}=1, \quad j_{1,3}(1)=\left\lfloor\frac{1}{3}\right\rfloor=0 ; \\
& \nu=2 \in \Delta_{1,3}^{(1)} \quad \Longrightarrow \quad a_{1,3}(2)=3\left\langle\frac{2}{3}\right\rangle=1, \quad j_{1,3}(2)=\left\lceil\frac{2}{3}\right\rceil=1 .
\end{aligned}
$$

При подстановке в формулу (3.6) возникают две структурированные суммы

$$
\begin{aligned}
& \mathfrak{S}_{1,3}^{d}(z ; 1)=2 z^{\lceil 1 / 3\rceil}(1-z)^{1-\lfloor 1 / 3\rfloor} \sum_{k=0}^{d} \frac{1}{3 k+1} C_{3 k+1}^{k}\left(z(1-z)^{2}\right)^{k}, \quad d \in \mathbb{N} \cup\{0\}, \\
& \mathfrak{S}_{1,3}^{d}(z ; 2)=2 z^{\lceil 2 / 3\rceil}(1-z)^{2-\lfloor 2 / 3\rfloor} \sum_{k=0}^{d} \frac{1}{3 k+2} C_{3 k+2}^{k+1}\left(z(1-z)^{2}\right)^{k}, \quad d \in \mathbb{N} \cup\{0\} .
\end{aligned}
$$

В последней (второй) сумме используем элементарное тождество $C_{3 k+2}^{k+1}=2 C_{3 k+2}^{k}$. Тогда обнаружится возможность общей записи

$$
\mathfrak{S}_{1,3}^{d}(z ; \nu)=2 z(1-z)^{\nu} \sum_{k=0}^{d} \frac{\nu}{3 k+\nu} C_{3 k+\nu}^{k}\left(z(1-z)^{2}\right)^{k}, \quad \nu=1,2, \quad d \in \mathbb{N} \cup\{0\} .
$$

Применив теперь правило (3.9), устанавливаем указанное прежде представление (3.14) для полиномов, образующих цепочку склеивания $B_{3 m+1}(f, z)=B_{3 m}(f, z)$. Затем правило $(3.10)$ при $r=2$ дает представление (3.15) для полиномов $B_{3 m+2}(f, z)$.

Итак, проверка конкретных представлений, анонсированных в разделе 3, завершена. Покажем теперь, что идея, использованная в примере 7.2 , допускает развитие, и полиномы Бернштейна для модулей вида $f(x)=|q x-1|$ при всех значениях $q \in \mathbb{N}, q \geqslant 3$, приводятся к особой унифицированной форме. Надо только сделать одно дополнительное преобразование в последней из структурированных сумм, отвечающей номеру $\nu=q-1$. Полностью разберем этот специальный случай.

Пример 7.3. Пусть $f(x)=|q x-1|$ на $[0,1]$. Здесь $p=1$, а $q \in \mathbb{N}$ и $q \geqslant 3$. Множество (7.1) содержит номера $\nu$ от 1 до $q-1$. Условие (7.2) дает выражение $\langle\nu / q\rangle \leqslant 1 / q$, выполненное, очевидно, лишь при $\nu=q-1$; соответственно, $\Delta_{1, q}^{(1)}=\{q-1\}$ и $\Delta_{1, q}^{(2)}=\{1, \ldots, q-2\}$. Подсчет величин (3.3) с учетом того, что $0<\nu / q<1$, дает следующие результаты:

$$
\begin{gathered}
\nu=1, \ldots, q-2 \in \Delta_{1, q}^{(2)} \Longrightarrow \quad a_{1, q}(\nu)=q\left\{\frac{\nu}{q}\right\}=\nu, \quad j_{1, q}(\nu)=\left\lfloor\frac{\nu}{q}\right\rfloor=0 \\
\nu=q-1 \in \Delta_{1, q}^{(1)} \Longrightarrow \quad a_{1, q}(q-1)=q\left\langle\frac{q-1}{q}\right\rangle=1, \quad j_{1, q}(q-1)=\left\lceil\frac{q-1}{q}\right\rceil=1 .
\end{gathered}
$$

В результате подстановки в $(3.6)$ образуется $(q-1)$ структурированная сумма, из которых $(q-2)$ суммы имеют вид

$$
\mathfrak{S}_{1, q}^{d}(z ; \nu)=2 z^{\lceil\nu / q\rceil}(1-z)^{\nu-\lfloor\nu / q\rfloor} \sum_{k=0}^{d} \frac{\nu}{q k+\nu} C_{q k+\nu}^{k}\left(z(1-z)^{q-1}\right)^{k}, \quad \nu=1, \ldots, q-2, \quad d \in \mathbb{N} \cup\{0\},
$$

и одна отдельная сумма вида

$$
\mathfrak{S}_{1, q}^{d}(z ; \nu)=2 z^{\lceil\nu / q\rceil}(1-z)^{\nu-\lfloor\nu / q\rfloor} \sum_{k=0}^{d} \frac{1}{q k+\nu} C_{q k+\nu}^{k+1}\left(z(1-z)^{q-1}\right)^{k}, \quad \nu=q-1, \quad d \in \mathbb{N} \cup\{0\} .
$$


Переписав последнюю сумму с учетом элементарного тождества $C_{q k+q-1}^{k+1}=(q-1) C_{q k+q-1}^{k}$, означающего, что $C_{q k+\nu}^{k+1}=\nu C_{q k+\nu}^{k}$ при $\nu=q-1$, получаем общую запись структурированных сумм

$$
\mathfrak{S}_{1, q}^{d}(z ; \nu)=2 z(1-z)^{\nu} \sum_{k=0}^{d} \frac{\nu}{q k+\nu} C_{q k+\nu}^{k}\left(z(1-z)^{q-1}\right)^{k}, \quad d \in \mathbb{N} \cup\{0\},
$$

справедливую при всех $\nu \in\{1, \ldots, q-1\}$, т.е. при всех $\nu$ из множества (7.1).

Применив теперь правило (3.9), получим

$$
B_{q m+1}(f, z)=B_{q m}(f, z)=1+(q-2) z-\sum_{\nu=1}^{q-1} 2 z(1-z)^{\nu} \sum_{k=0}^{m-1} \frac{\nu}{q k+\nu} C_{q k+\nu}^{k}\left(z(1-z)^{q-1}\right)^{k}
$$

для полиномов, образующих цепочку склеиваний. Для прочих остатков $r=2, \ldots, q-1$ по формуле (3.10) имеем

$$
\begin{aligned}
B_{q m+r}(f, z)=1+(q-2) z-\sum_{\nu=1}^{r-1} 2 z(1-z)^{\nu} \sum_{k=0}^{m} & \frac{\nu}{q k+\nu} C_{q k+\nu}^{k}\left(z(1-z)^{q-1}\right)^{k}- \\
& -\sum_{\nu=r}^{q-1} 2 z(1-z)^{\nu} \sum_{k=0}^{m-1} \frac{\nu}{q k+\nu} C_{q k+\nu}^{k}\left(z(1-z)^{q-1}\right)^{k} .
\end{aligned}
$$

Вводя символ $\varepsilon_{m}(\nu, r)$ по правилу (3.2), устанавливаем компактную запись разложения

$$
B_{q m+r}(f, z)=1+(q-2) z-\sum_{\nu=1}^{q-1} 2 z(1-z)^{\nu} \sum_{k=0}^{\varepsilon_{m}(\nu, r)} \frac{\nu}{q k+\nu} C_{q k+\nu}^{k}\left(z(1-z)^{q-1}\right)^{k}
$$

действующую при всех $m \in \mathbb{N}$ и $r=0,1, \ldots, q-1$. Наличие унифицированной формулы $(7.5)$ существенно облегчает изучение полиномов Бернштейна для функции $f(x)=|q x-1|$ с точкой излома $x=1 / q$.

Разумеется, аналогичные эффекты должны действовать для зеркально симметричного модуля с точкой излома $x=(q-1) / q$. Для полноты изложения выведем соответствующее разложение Поповичу из базовой формулы (3.1), хотя, конечно, можно было бы просто воспользоваться формулой (7.5) и сделать там замену $z$ на $1-z$.

Пример 7.4. Пусть $f(x)=|q x-q+1|$ на $[0,1]$. Здесь $p=q-1, q \in \mathbb{N}, q \geqslant 3$. Множество (7.1) содержит номера $\nu$ от 1 до $q-1$. Условие (7.2) принимает вид

$$
\left\langle\frac{(q-1) \nu}{q}\right\rangle \leqslant \frac{q-1}{q}
$$

Так как $\nu \in \mathbb{N}$ и $0<\nu / q<1$, то по определению антидробной части из (2.4) имеем

$$
\left\langle\frac{(q-1) \nu}{q}\right\rangle=\left\langle\nu-\frac{\nu}{q}\right\rangle=\frac{\nu}{q}
$$

т.е. (7.6) переходит в неравенство $\nu \leqslant q-1$, выполненное при всех $\nu \in\{1, \ldots, q-1\}$; соответственно, $\Delta_{q-1, q}^{(1)}=\{1, \ldots, q-1\}$ и $\Delta_{q-1, q}^{(2)}=\varnothing$. Подсчет величин (3.3) дает следующий результат:

$$
\nu=1, \ldots, q-1 \in \Delta_{q-1, q}^{(1)} \quad \Longrightarrow \quad a_{q-1, q}(\nu)=q\left\langle\frac{(q-1) \nu}{q}\right\rangle=\nu, \quad j_{q-1, q}(\nu)=\left\lceil\frac{(q-1) \nu}{q}\right\rceil=\nu .
$$

При подстановке данных в (3.1) учтем также, что

$$
\left.z^{\lceil p \nu / q\rceil}(1-z)^{\nu-\lfloor p \nu / q\rfloor}\right|_{p=q-1}=z^{\lceil(q-1) \nu / q\rceil}(1-z)^{\nu-\lfloor(q-1) \nu / q\rfloor}=z^{\nu}(1-z) .
$$


Тем самым, для случая $f(x)=|q x-q+1|$ формула (3.1) приводит к представлению

$$
B_{q m+r}(f, z)=q-1-(q-2) z-\sum_{\nu=1}^{q-1} 2 z^{\nu}(1-z) \sum_{k=0}^{\varepsilon_{m}(\nu, r)} \frac{\nu}{q k+\nu} C_{q k+\nu}^{(q-1) k+\nu}\left(z^{q-1}(1-z)\right)^{k}
$$

верному при всех $m \in \mathbb{N}$ и $r=0,1, \ldots, q-1$. Остается заметить, что $C_{q k+\nu}^{(q-1) k+\nu}=C_{q k+\nu}^{k}$, и записать окончательный итог в виде

$$
B_{q m+r}(f, z)=q-1-(q-2) z-\sum_{\nu=1}^{q-1} 2 z^{\nu}(1-z) \sum_{k=0}^{\varepsilon_{m}(\nu, r)} \frac{\nu}{q k+\nu} C_{q k+\nu}^{k}\left(z^{q-1}(1-z)\right)^{k}
$$

при всех $m \in \mathbb{N}$ и $r=0,1, \ldots, q-1$. Как и ожидалось, полиномы $(7.5)$ и (7.7) оказались «зеркально симметричными», переходя друг в друга при замене $z$ на $1-z$.

Рассмотренные примеры 7.1-7.4 дают регулярные выражения для разложений Поповичу, где все параметры изменяются неким «правильным» образом. Во многих других случаях так просто не получается из-за нетривиального разброса индекса $\nu$ по множествам $\Delta_{p, q}^{(1)}, \Delta_{p, q}^{(2)}$ и общей непредсказуемости при вычислении величин (3.3). Подробно разберем один типичный пример.

Пример 7.5. Пусть $f(x)=|5 x-2|$ на $[0,1]$. Здесь $p=2, q=5$ с множеством $\Delta=\{1,2,3,4\}$. Условие (7.2) дает выражение $\langle 2 \nu / 5\rangle \leqslant 2 / 5$, выполненное при $\nu=2,4$. Отсюда $\Delta_{2,5}^{(1)}=\{2,4\}$ и $\Delta_{2,5}^{(2)}=\{1,3\}$. Подсчет величин $a_{2,5}(\nu), j_{2,5}(\nu)$ по формулам (3.3) приводит к следующим значениям:

$$
\begin{aligned}
& \nu=1 \in \Delta_{2,5}^{(2)} \quad \Longrightarrow \quad a_{2,5}(1)=5\left\{\frac{2}{5}\right\}=2, \quad j_{2,5}(1)=\left\lfloor\frac{2}{5}\right\rfloor=0 ; \\
& \nu=2 \in \Delta_{2,5}^{(1)} \quad \Longrightarrow \quad a_{2,5}(2)=5\left\langle\frac{4}{5}\right\rangle=1, \quad j_{2,5}(2)=\left\lceil\frac{4}{5}\right\rceil=1 ; \\
& \nu=3 \in \Delta_{2,5}^{(2)} \quad \Longrightarrow \quad a_{2,5}(3)=5\left\{\frac{6}{5}\right\}=1, \quad j_{2,5}(3)=\left\lfloor\frac{6}{5}\right\rfloor=1 ; \\
& \nu=4 \in \Delta_{2,5}^{(1)} \quad \Longrightarrow \quad a_{2,5}(4)=5\left\langle\frac{8}{5}\right\rangle=2, \quad j_{2,5}(4)=\left\lceil\frac{8}{5}\right\rceil=2 .
\end{aligned}
$$

При подстановке в (3.6) возникают четыре структурированные суммы

$$
\begin{aligned}
& \mathfrak{S}_{2,5}^{d}(z ; 1)=2 z^{\lceil 2 / 5\rceil}(1-z)^{1-\lfloor 2 / 5\rfloor} \sum_{k=0}^{d} \frac{2}{5 k+1} C_{5 k+1}^{2 k}\left(z^{2}(1-z)^{3}\right)^{k}, \\
& \mathfrak{S}_{2,5}^{d}(z ; 2)=2 z^{\lceil 4 / 5\rceil}(1-z)^{2-\lfloor 4 / 5\rfloor} \sum_{k=0}^{d} \frac{1}{5 k+2} C_{5 k+2}^{2 k+1}\left(z^{2}(1-z)^{3}\right)^{k}, \\
& \mathfrak{S}_{2,5}^{d}(z ; 3)=2 z^{\lceil 6 / 5\rceil}(1-z)^{3-\lfloor 6 / 5\rfloor} \sum_{k=0}^{d} \frac{1}{5 k+3} C_{5 k+3}^{2 k+1}\left(z^{2}(1-z)^{3}\right)^{k}, \\
& \mathfrak{S}_{2,5}^{d}(z ; 4)=2 z^{\lceil 8 / 5\rceil}(1-z)^{4-\lfloor 8 / 5\rfloor} \sum_{k=0}^{d} \frac{2}{5 k+4} C_{5 k+4}^{2 k+2}\left(z^{2}(1-z)^{3}\right)^{k} .
\end{aligned}
$$


Обрабатывая найденные выражения и подставляя их в формулу (3.1) (или в эквивалентные формулы (3.9), (3.10)), получаем нужное разложение

$$
\begin{aligned}
& B_{5 m+r}(f, z)=2+z-2 z(1-z) \sum_{k=0}^{\varepsilon_{m}(1, r)} \frac{2}{5 k+1} C_{5 k+1}^{2 k}\left(z^{2}(1-z)^{3}\right)^{k}- \\
&-2 z(1-z)^{2} \sum_{k=0}^{\varepsilon_{m}(2, r)} \frac{1}{5 k+2} C_{5 k+2}^{2 k+1}\left(z^{2}(1-z)^{3}\right)^{k}-2 z^{2}(1-z)^{2} \sum_{k=0}^{\varepsilon_{m}(3, r)} \frac{1}{5 k+3} C_{5 k+3}^{2 k+1}\left(z^{2}(1-z)^{3}\right)^{k}- \\
&-2 z^{2}(1-z)^{3} \sum_{k=0}^{\varepsilon_{m}(4, r)} \frac{2}{5 k+4} C_{5 k+4}^{2 k+2}\left(z^{2}(1-z)^{3}\right)^{k}
\end{aligned}
$$

при всех $m \in \mathbb{N}$ и $r=0,1,2,3,4$. Здесь значения $\varepsilon_{m}(\nu, r)$ при $\nu=1,2,3,4$ вычисляются по правилу (3.2). Другими словами,

$$
\begin{aligned}
& r=0,1 \quad \Longrightarrow \quad \varepsilon_{m}(\nu, r)=m-1 \text { при всех } \nu=1,2,3,4 ; \\
& r=2,3,4 \quad \Longrightarrow \quad \varepsilon_{m}(\nu, r)=m \text { при } \nu=1, \ldots, r-1 \text { и } \varepsilon_{m}(\nu, r)=m-1 \text { при } \nu=r, \ldots, 4 .
\end{aligned}
$$

Например, для полиномов из цепочки склеиваний имеем выражение

$$
\begin{aligned}
& B_{5 m+1}(f, z)=B_{5 m}(f, z)=2+z-2 z(1-z) \sum_{k=0}^{m-1} \frac{2}{5 k+1} C_{5 k+1}^{2 k}\left(z^{2}(1-z)^{3}\right)^{k}- \\
&-2 z(1-z)^{2} \sum_{k=0}^{m-1} \frac{1}{5 k+2} C_{5 k+2}^{2 k+1}\left(z^{2}(1-z)^{3}\right)^{k}-2 z^{2}(1-z)^{2} \sum_{k=0}^{m-1} \frac{1}{5 k+3} C_{5 k+3}^{2 k+1}\left(z^{2}(1-z)^{3}\right)^{k}- \\
&-2 z^{2}(1-z)^{3} \sum_{k=0}^{m-1} \frac{2}{5 k+4} C_{5 k+4}^{2 k+2}\left(z^{2}(1-z)^{3}\right)^{k}
\end{aligned}
$$

при всех $m \in \mathbb{N}$.

Вообще же, переход от полинома $B_{n}(f, z)$ к следующему полиному $B_{n+1}(f, z)$ в данном примере регулируется правилами

$$
\begin{aligned}
& B_{5 m+2}(f, z)=B_{5 m+1}(f, z)-2 z(1-z) \frac{2}{5 m+1} C_{5 m+1}^{2 m}\left(z^{2}(1-z)^{3}\right)^{m} \\
& B_{5 m+3}(f, z)=B_{5 m+2}(f, z)-2 z(1-z)^{2} \frac{1}{5 m+2} C_{5 m+2}^{2 m+1}\left(z^{2}(1-z)^{3}\right)^{m} \\
& B_{5 m+4}(f, z)=B_{5 m+3}(f, z)-2 z^{2}(1-z)^{2} \frac{1}{5 m+3} C_{5 m+3}^{2 m+1}\left(z^{2}(1-z)^{3}\right)^{m} \\
& B_{5 m+5}(f, z)=B_{5 m+4}(f, z)-2 z^{2}(1-z)^{3} \frac{2}{5 m+4} C_{5 m+4}^{2 m+2}\left(z^{2}(1-z)^{3}\right)^{m} \\
& B_{5 m+6}(f, z)=B_{5 m+5}(f, z) .
\end{aligned}
$$

Согласно общей формуле (6.2) эти рекуррентные соотношения действуют при всех $m \in \mathbb{N} \cup\{0\}$. В их записи участвуют найденные значения $a_{2,5}(\nu), j_{2,5}(\nu)$.

Нет сомнений, что в рамках подобной схемы можно разложить полиномы Бернштейна для любого рационального модуля вида (1.2), и дальнейшие примеры, должно быть, излишни.

Подведем итог. Несмотря на необходимость некоторых подготовительных расчетов и формальную громоздкость возникающих выражений, рассмотренные примеры наглядно показывают главное преимущество разложений Поповичу по сравнению с исходным определением полиномов Бернштейна (1.1). Как видно из формул (3.11)-(3.15), (7.5), (7.7)-(7.9), коэффициенты разложений не зависят от номера полинома Бернштейна, и при увеличении номера происходит лишь поочередное добавление слагаемых, каждое из которых имеет вполне конкретную структуру и органично вписывается в ту или иную структурированную сумму. Фактически полиномы Бернштейна для функции $f(x)=|q x-p|$ превращаются в набор из $(q-1)$ частичной сумм для $(q-1)$ 
функционального ряда. Это сближает теорию полиномов Бернштейна с теорией степенных рядов, позволяя точно находить комплексную область сходимости полиномов Бернштейна для любого рационального модуля вида (1.2) и оценивать скорость стремления полиномов к соответствующей предельной функции. Элементарные приемы обеспечивают вполне законченные результаты. Продемонстрируем открывающиеся возможности.

8. Сходимость полиномов и компакты Канторовича. Уже на ранних этапах развития теории полиномов Бернштейна в работах Канторовича [7] и Райта [61] было замечено, что при определенных требованиях аналитичности порождающей функции $f \in C[0,1]$ сходимость полиномов Бернштейна распространяется в некоторую область комплексной плоскости, связанную с имеющимися особенностями функции $f$. Слово «область» понимается здесь весьма расширительно, означая всё множество сходимости или какую-то его значимую часть. Высоко оценив идеи Л. В. Канторовича, исследования в данном направлении продолжил и развил уже сам С. Н. Бернштейн в двух ёмких, технически трудных работах $[1,2]$. Адаптированное изложение результатов Бернштейна (и, отчасти, Канторовича) дано в четвертой главе книги [49]. Ряд дополнительных сведений и ссылок по теме можно найти в недавней монографии [42].

Отвлекаясь от многих деталей и сложностей, выделим два базовых принципа, восходящих к работе Канторовича [7].

Принцип 1. Пусть функция $f \in C[0,1]$ является сужением на $[0,1]$ целой функции $f(z)$. Тогда последовательность полиномов Бернштейна (1.1) при $n \rightarrow \infty$ сходится к $f(z)$ равномерно на любом компакте в плоскости $\mathbb{C}$ (см. [7, теорема 1], а также [11, с. 254-256]).

Принцип 2. Пусть $f \in C[0,1]$, причем $f(x) \equiv \varphi_{1}(x)$ на отрезке $[0, c] \subset[0,1)$ и $f(x) \equiv \varphi_{2}(x)$ на отрезке $[c, 1] \subset(0,1]$, где $\varphi_{1}(z), \varphi_{2}(z)$ - целые функции в плоскости $\mathbb{C}$. Тогда последовательность полиномов Бернштейна (1.1) при $n \rightarrow \infty$ сходится в области, ограниченной кривой

$$
\Lambda_{c}:\left|\frac{z}{c}\right|^{c}\left|\frac{1-z}{1-c}\right|^{1-c}=1
$$

в левой петле - к функции $\varphi_{1}(z)$, а в правой петле - к функции $\varphi_{2}(z)$.

Лемнискатоподобные кривые вида (8.1), где значение $c \in(0,1)$ выступает в роли параметра, играют ключевую роль при описании области сходимости полиномов Бернштейна для всякой порождающей функции $f \in C[0,1]$, «составленной» из нескольких аналитических кусков. Собственно, именно этот факт и был открыт в [7, теорема 3]. Поэтому всякую кривую (8.1) с фиксированным значением $c \in(0,1)$ будем называть лемнискатой Канторовича, а компакт, ограниченный такой кривой, т.е. множество

$$
K_{c} \equiv\left\{z \in \mathbb{C}:\left|\frac{z}{c}\right|^{c}\left|\frac{1-z}{1-c}\right|^{1-c} \leqslant 1\right\},
$$

называем компактом Канторовича.

Сформулированный выше принцип 2 несколько отличается от оригинальной версии [7]. Доказательство результата в нужной нам форме получается сочетанием принципа 1 и теоремы 4.1.3 из монографии [49].

Отметим, кстати, что ввиду сложности темы формулировки в классических работах упомянутого цикла не всегда обладают должной отчётливостью. Среди прочего часто остается неясным, сохранится ли сходимость полиномов Бернштейна на границе обсуждаемой области и будет ли наблюдаться расходимость во всех точках вне области. Также почти не изучался вопрос о скорости сходимости полиномов Бернштейна $B_{n}(f, z)$ для комплексных значений $z$ вне основного отрезка $[0,1]$.

Теперь же, используя разработанный аппарат обобщенных разложений Поповичу, можно получить полную картину сходимости полиномов Бернштейна в плоскости $\mathbb{C}$ для любого рационального модуля (1.2) при ограничениях (2.1). 


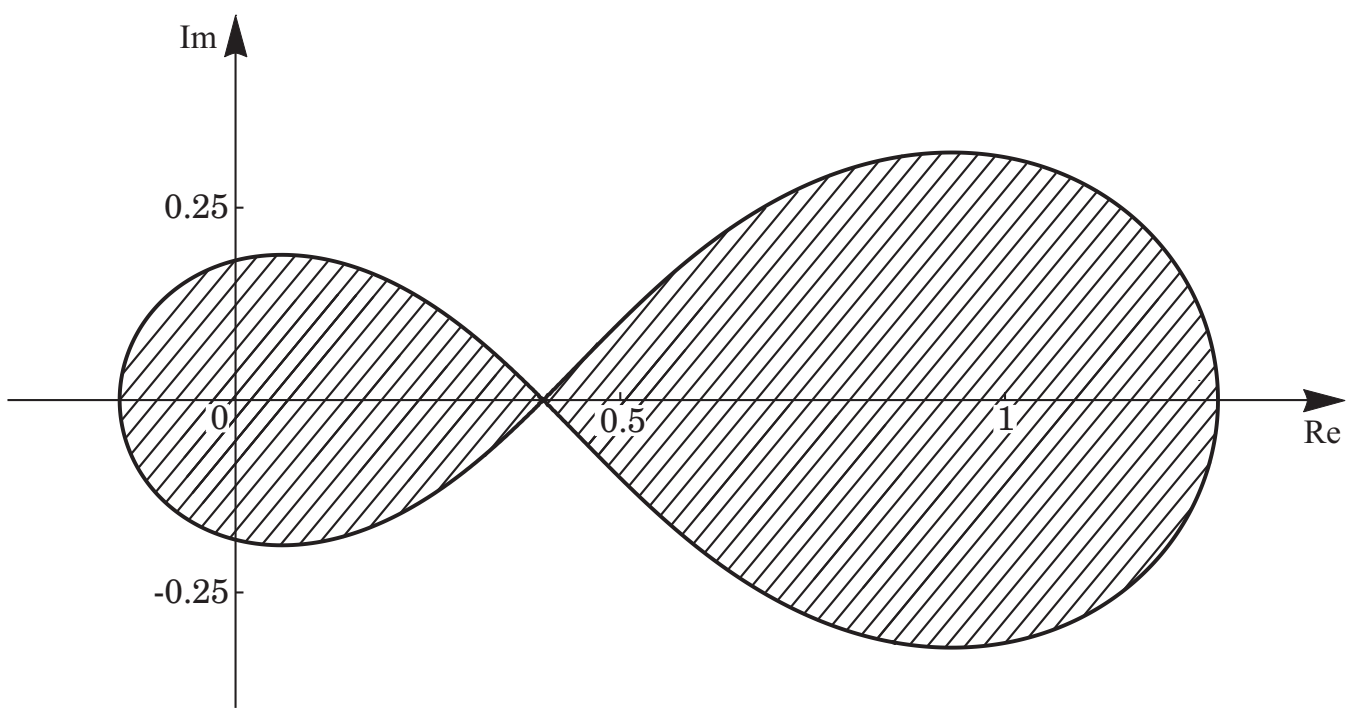

Рис. 1. Лемниската Канторовича и соответствующий компакт, построенные по формулам (8.3) и (8.4) при $p=2, q=5$, т.е. для функции $f(x)=|5 x-2|$.

В этом случае $f(x)=p-q x$ при $x \in[0, p / q]$ и $f(x)=q x-p$ при $x \in[p / q, 1]$ с разделительной точкой излома $c=p / q$. Ситуация полностью подпадает под сформулированный выше принцип 2. Уравнение (8.1) для лемнискаты Канторовича приводится к виду

$$
\Lambda_{p / q} \equiv \Lambda_{p, q}: \frac{q^{q}}{p^{p}(q-p)^{q-p}}\left|z^{p}(1-z)^{q-p}\right|=1 .
$$

Лемниската (8.3) ограничивает компакт Канторовича

$$
K_{p / q} \equiv K_{p, q}=\left\{z \in \mathbb{C}: \frac{q^{q}}{p^{p}(q-p)^{q-p}}\left|z^{p}(1-z)^{q-p}\right| \leqslant 1\right\}
$$

Между прочим, уравнение кривой (8.3) полностью соответствует стандартному определению лемнискаты как линии уровня алгебраического полинома переменной $z \in \mathbb{C}$ (см. [9, с. 313-316]). Компакт $K_{p, q}$ состоит из левой петли $K_{p, q}^{(1)}$ и правой петли $K_{p, q}^{(2)}$, определяемых по правилам

$$
K_{p, q}^{(1)} \equiv K_{p, q} \cap\{\operatorname{Re} z \leqslant p / q\}, \quad K_{p, q}^{(2)} \equiv K_{p, q} \cap\{\operatorname{Re} z \geqslant p / q\} .
$$

Ясно, что $K_{p, q}^{(1)} \cup K_{p, q}^{(2)}=K_{p, q}$ и $K_{p, q}^{(1)} \cap K_{p, q}^{(2)}=\{p / q\}$. Все точки основного отрезка [0,1] попадают строго внутрь $K_{p, q}$, за исключением одной лишь точки $c=p / q$-точки самопересечения лемнискаты (8.3).

Наглядное представление о происходящем на примере рационального модуля $f(x)=|5 x-2|$, т.е. при $p=2$ и $q=5$, дает рис. 1 .

Точное описание множества сходимости полиномов Бернштейна для всякого рационального модуля вида (1.2) выглядит следующим образом.

Теорема 8.1. Пусть $B_{n}(f, z)$ - полиномы Бернштейна (1.1) от функиии (1.2) при соглашениях (2.1). Тогда $B_{n}(f, z) \rightarrow \varphi(z)$ при $n \rightarrow \infty$ равномерно на компакте $K_{p, q}$ из формуль (8.4). Здесь

$$
\varphi(z) \equiv\left\{\begin{array}{ll}
p-q z, & z \in K_{p, q}^{(1)}, \\
q z-p, & z \in K_{p, q}^{(2)},
\end{array} \quad \varphi(x)=f(x) \text { nрu } x \in[0,1] .\right.
$$

Для любой внешней точки $z \in \mathbb{C} \backslash K_{p, q}$ последовательность $B_{n}(f, z)$ расходится, т.е. не имеет конечного предела в $\mathbb{C}$. 
Ключевую роль в доказательстве теоремы 8.1 играет один результат об оценках последовательных разностей полиномов Бернштейна. Для его формулировки введем обозначения

$$
\begin{gathered}
M_{p, q}(\nu) \equiv \frac{(q+\nu-1)^{\nu}}{(\min \{p, q-p\})^{\nu+1}} \sqrt{\frac{2 p(q-p)}{q \pi}} \max _{z \in \Lambda_{p, q}}\left|z^{\lceil p \nu / q\rceil}(1-z)^{\nu-\lfloor p \nu / q\rfloor}\right|>0, \\
L_{p, q}(\nu) \equiv \frac{7}{8} \frac{q^{\nu-1}}{(\max \{p+\nu, q-p+\nu\})^{\nu}} \sqrt{\frac{2 q}{p(q-p) \pi}} \min _{z \in \Lambda_{p, q}}\left|z^{\lceil p \nu / q\rceil}(1-z)^{\nu-\lfloor p \nu / q\rfloor}\right|>0,
\end{gathered}
$$

где $\nu=1, \ldots, q-1$, а $\Lambda_{p, q}$-лемниската Канторовича (8.3). Введем также важную величину, точнее, полином

$$
T_{p, q}(z) \equiv \frac{q^{q}}{p^{p}(q-p)^{q-p}} z^{p}(1-z)^{q-p}, \quad z \in \mathbb{C},
$$

определяющий лемнискату (8.3) уравнением $\left|T_{p, q}(z)\right|=1$. Будем называть $T_{p, q}(z)$ первичным полиномом Канторовича. Зафиксируем для ссылок связь

$$
\left\{\begin{array}{l}
\left|T_{p, q}(z)\right|<1, \quad z \in \operatorname{int} K_{p, q}, \\
\left|T_{p, q}(z)\right|=1, \quad z \in \partial K_{p, q}=\Lambda_{p, q}, \\
\left|T_{p, q}(z)\right|>1, \quad z \in \mathbb{C} \backslash K_{p, q},
\end{array}\right.
$$

между полиномом (8.9) и порожденными им лемнискатой (8.3) и компактом (8.4).

Основываясь на представлении (6.2) из леммы 6.1, можно установить следующий результат.

Лемма 8.1. Для полиномов Бернштейна (1.1) от функиии (1.2) при соглашениях (2.1) верны оченки

$$
\begin{aligned}
& \left|B_{q k+\nu+1}(f, z)-B_{q k+\nu}(f, z)\right| \leqslant M_{p, q}(\nu) \frac{1}{k^{3 / 2}}\left|T_{p, q}(z)\right|^{k}, \quad z \in K_{p, q}, \\
& \left|B_{q k+\nu+1}(f, z)-B_{q k+\nu}(f, z)\right| \geqslant L_{p, q}(\nu) \frac{1}{k^{3 / 2}}\left|T_{p, q}(z)\right|^{k}, \quad z \in \mathbb{C} \backslash K_{p, q},
\end{aligned}
$$

с компактом $K_{p, q}$ из (8.4) и полиномом $T_{p, q}(z)$ из (8.9). Здесъ $k \in \mathbb{N}, \nu=1, \ldots, q-1$, а коэфбициенты $M_{p, q}(\nu)$ и $L_{p, q}(\nu)$ определены формулами (8.7) и (8.8) соответственно.

Отсюда, ввиду связи (8.10), ясно, что разность между двумя последовательными полиномами $B_{q k+\nu+1}(f, z)$ и $B_{q k+\nu}(f, z)$ внутри компакта $K_{p, q}$ при больших значениях $n=q k+\nu$ становится экспоненциально малой, а вне компакта - экспоненциально большой. Лемма 8.1 позволяет также оценить скорость сходимости изучаемых полиномов Бернштейна на компакте $K_{p, q}$ к соответствующей предельной функции (8.6). Для формулировки результата введем обозначение

$$
M_{p, q} \equiv \sum_{\nu=1}^{q-1} M_{p, q}(\nu)>0
$$

со значениями $M_{p, q}(\nu)$ из формулы $(8.7)$.

Теорема 8.2. Пусть $B_{n}(f, z)$ - полиномы Бернштейна (1.1) от функиии (1.2) при соглашениях (2.1). Пусть уклонения полиномов от предельной функиии (8.6) заданы формулой

$$
R_{n}(f, z) \equiv B_{n}(f, z)-\varphi(z), \quad z \in K_{p, q}, \quad n \in \mathbb{N} .
$$

Тогда при всех $z \in \operatorname{int} K_{p, q}$, т.е. внутри компакта Канторовича (8.4), имеем оценку

$$
\left|R_{q m+r}(f, z)\right| \leqslant \frac{M_{p, q}}{1-\left|T_{p, q}(z)\right|} \frac{\left|T_{p, q}(z)\right|^{m}}{m^{3 / 2}}, \quad m \in \mathbb{N}, \quad r=0,1, \ldots, q-1,
$$

с величинами $T_{p, q}(z), M_{p, q}$ из формул (8.9) и (8.13) соответственно. Если же $z \in \partial K_{p, q}=\Lambda_{p, q}$, mo

$$
\left|R_{q m+r}(f, z)\right| \leqslant \frac{2 M_{p, q}}{\sqrt{m}}\left(1+\frac{1}{2 m}\right), \quad m \in \mathbb{N}, \quad r=0,1, \ldots, q-1,
$$

с величиной $M_{p, q}$ из формуль (8.13). 
Для того чтобы избежать чрезмерной детализации, мы даем оценки (8.15) и (8.16) в несколько огрубленной форме - без учета влияния остатка $r$ в номере $n=q m+r$ полинома Бернштейна. Тем не менее, теорема 8.2 показывает главное. Как видно из оценки (8.15), внутри компакта $K_{p, q}$ скорость сходимости полиномов Бернштейна к своей предельной функции является экспоненциальной (ибо $\left|T_{p, q}(z)\right|<1$ при $z \in \operatorname{int} K_{p, q}$ ). На границе же действует другая, более слабая степенная оценка (8.16). Эти качественные различия весьма точно отражают суть дела и соответствуют нашим прежним прогнозам в [25].

План доказательства перечисленных результатов будет следующий. Сначала, в разделе 9, мы получим нужные оценки биномиальных коэффициентов, фигурирующих в формуле (6.2). Затем, в следующем разделе 10, докажем лемму 8.1 об оценках разностей полиномов Бернштейна и выведем из нее основные теоремы 8.1 и 8.2 .

9. Оценки биномиальных коэффициентов. Задачи об оценках факториальных величин, в том числе биномиальных коэффициентов, давно интересуют математиков. Исходным результатом была знаменитая формула Валлиса (1656 г.), точный исторический вид которой (см. [33, с. $152-155]$ или $[41$, с. 12$])$ означал равенство

$$
\frac{4}{\pi}=\frac{3}{2} \cdot \frac{3}{4} \cdot \frac{5}{4} \cdot \frac{5}{6} \cdot \frac{7}{6} \cdot \frac{7}{8} \cdot \frac{9}{8} \cdot \frac{9}{10} \cdots \frac{2 k-1}{2 k-2} \cdot \frac{2 k-1}{2 k} \cdots .
$$

Более удобно группировать попарно

$$
\frac{4}{\pi}=\frac{3 \cdot 3}{2 \cdot 4} \cdot \frac{5 \cdot 5}{4 \cdot 6} \cdot \frac{7 \cdot 7}{6 \cdot 8} \cdot \frac{9 \cdot 9}{8 \cdot 10} \cdots \frac{(2 k-1) \cdot(2 k-1)}{(2 k-2) \cdot(2 k)} \cdots .
$$

Обрабатывая бесконечное произведение (9.1), получаем запись

$$
\frac{1}{\pi}=\lim _{k \rightarrow \infty} k\left[\frac{(2 k-1) ! !}{(2 k) ! !}\right]^{2}=\lim _{k \rightarrow \infty} k\left[2^{-2 k} C_{2 k}^{k}\right]^{2}
$$

и равносильную ей асимптотику

$$
2^{-2 k} C_{2 k}^{k} \sim \frac{1}{\sqrt{k \pi}}, \quad k \rightarrow \infty .
$$

Последнюю естественно называть асимптотикой Валлиса. Поскольку все сомножители в (9.1) больше единицы, то сходимость к пределу (9.2) происходит монотонно снизу. Соответственно,

$$
2^{-2 k} C_{2 k}^{k}<\frac{1}{\sqrt{k \pi}}, \quad k \in \mathbb{N}
$$

«Неасимптотическое» неравенство (9.4) подкрепляет асимптотику Валлиса (9.3). Дополнительную информацию по оценкам центрального биномиального коэффициента см. в [16, с. 59-61] или [46].

Общую основу подобных результатов дает формула Стирлинга, простейший асимптотический вид которой общеизвестен:

$$
n ! \sim \sqrt{2 n \pi}\left(\frac{n}{e}\right)^{n}, \quad n \rightarrow \infty .
$$

Традиционно считается, что для доказательства формулы Стирлинга нужна какая-либо версия формулы Валлиса (см., например, [29, п. 406]). Однако возможны и другие, независимые обоснования для (9.5) (см. [6,15]), позволяющие корректно применять саму формулу Стирлинга к выводу изначальной асимптотики (9.3).

Факториальные величины отличаются, как правило, регулярным поведением, и для них найдено много оценок, до сих пор активно уточняемых. Одно из недавних достижений [58] удачно укладывается в контекст нашего исследования.

Напомним, что нам нужны оценки биномиального коэффициента $C_{q k+\nu}^{p k+j_{p, q}(\nu)}$, возникшего при выводе формулы (6.2). Начнем с базового случая - «невозмущённого» коэффициента $C_{q k}^{p k}$. Всюду в данном разделе считаем, что $p, q$ - фиксированные натуральные значения, удовлетворяющие ограничениям (2.1). В роли переменной выступает $k \in \mathbb{N}$. 
Из формулы Стирлинга (9.5) следует асимптотика (ср. [14, с. 105])

$$
C_{q k}^{p k} \sim \sqrt{\frac{q}{2 p(q-p) k \pi}}\left(\frac{q^{q}}{p^{p}(q-p)^{q-p}}\right)^{k}, \quad k \rightarrow \infty .
$$

Недавно в [58] на основе идей из $[55,56]$ установлены двусторонние оценки для $C_{q k}^{p k}$, согласованные с асимптотикой (9.6). Показано (см. следствие 2.3 к теореме 2.2 в [58]), что

$$
\exp \left(-\frac{q^{2}-p q+p^{2}}{12 p q(q-p) k}\right) \sqrt{\frac{q}{2 p(q-p) k \pi}}<\left(\frac{q^{q}}{p^{p}(q-p)^{q-p}}\right)^{-k} C_{q k}^{p k}<\sqrt{\frac{q}{2 p(q-p) k \pi}}
$$

при всех $k \in \mathbb{N}$. Когда $p=1, q=2$, формула (9.7) содержит в себе прежнюю оценку сверху (9.4). Чуть загрубим нижнюю границу в (9.7) с учетом того, что $e^{-u}>1-u$ при $u>0$. Имеем

$$
\left(1-\frac{q^{2}-p q+p^{2}}{12 p q(q-p) k}\right) \sqrt{\frac{q}{2 p(q-p) k \pi}}<\left(\frac{q^{q}}{p^{p}(q-p)^{q-p}}\right)^{-k} C_{q k}^{p k}<\sqrt{\frac{q}{2 p(q-p) k \pi}}
$$

снова при всех $k \in \mathbb{N}$.

Принимая во внимание ограничения (2.1), заметим, что

$$
\frac{q^{2}-p q+p^{2}}{p q(q-p)}=\frac{1}{p}+\frac{p}{q(q-p)} \leqslant \frac{1}{p}+\frac{p}{(p+1) \cdot 1}=\frac{p^{2}+p+1}{p(p+1)}=1+\frac{1}{p(p+1)} \leqslant 1+\frac{1}{1 \cdot 2}=\frac{3}{2} .
$$

Поэтому из (9.8) следует, что

$$
\left(1-\frac{1}{8 k}\right) \sqrt{\frac{q}{2 p(q-p) k \pi}}<\left(\frac{q^{q}}{p^{p}(q-p)^{q-p}}\right)^{-k} C_{q k}^{p k}<\sqrt{\frac{q}{2 p(q-p) k \pi}}, \quad k \in \mathbb{N} .
$$

Поскольку поддержание асимптотики (9.6) сейчас не принципиально, еще ослабим нижнюю границу в (9.9), заменив там левую скобку на $7 / 8$. Затем, разделив на $k$, окончательно получаем

$$
\frac{7}{8} \sqrt{\frac{q}{2 p(q-p) \pi}} \frac{1}{k^{3 / 2}}<\left(\frac{q^{q}}{p^{p}(q-p)^{q-p}}\right)^{-k} \frac{C_{q k}^{p k}}{k}<\sqrt{\frac{q}{2 p(q-p) \pi}} \frac{1}{k^{3 / 2}}, \quad k \in \mathbb{N} .
$$

Этого результата для нас достаточно.

Установим теперь одно общее утверждение, позволяющее сравнивать «возмущённые» коэффициенты $C_{q k+\nu}^{p k+j_{p, q}(\nu)}$ со стандартными $C_{q k}^{p k}$.

Лемма 9.1. Пусть $j-$ целое число, причем $0 \leqslant j \leqslant \nu$, где $\nu \in \mathbb{N}$. Тогда

$$
\left(\frac{q}{\max \{p+\nu, q-p+\nu\}}\right)^{\nu} \leqslant \frac{q k}{q k+\nu} \frac{C_{q k+\nu}^{p k+j}}{C_{q k}^{p k}} \leqslant\left(\frac{q+\nu-1}{\min \{p, q-p\}}\right)^{\nu}, \quad k \in \mathbb{N} .
$$

Доказательство. Разберем возможный случай $0<j<\nu$. Запишем представление

$$
\frac{q k}{q k+\nu} \frac{C_{q k+\nu}^{p k+j}}{C_{q k}^{p k}}=\frac{q k(q k+1) \ldots(q k+\nu-1)}{(p k+1) \ldots(p k+j) \cdot((q-p) k+1) \ldots((q-p) k+\nu-j)} .
$$

Оценим эту величину сверху:

$$
\frac{q k}{q k+\nu} \frac{C_{q k+\nu}^{p k+j}}{C_{q k}^{p k}} \leqslant \frac{(q k+\nu-1)^{\nu}}{(p k)^{j}((q-p) k)^{\nu-j}}=\frac{\left(q+\frac{\nu-1}{k}\right)^{\nu}}{p^{j}(q-p)^{\nu-j}} \leqslant \frac{(q+\nu-1)^{\nu}}{p^{j}(q-p)^{\nu-j}} \leqslant \frac{(q+\nu-1)^{\nu}}{(\min \{p, q-p\})^{\nu}} .
$$

Но это и есть верхняя оценка в (9.11). 
Вновь пользуясь (9.12), дадим оценку снизу:

$$
\begin{gathered}
\frac{q k}{q k+\nu} \frac{C_{q k+\nu}^{p k+j}}{C_{q k}^{p k}} \geqslant \frac{(q k)^{\nu}}{(p k+j)^{j}((q-p) k+\nu-j)^{\nu-j}}=\frac{q^{\nu}}{\left(p+\frac{j}{k}\right)^{j}\left(q-p+\frac{\nu-j}{k}\right)^{\nu-j}} \geqslant \\
\geqslant \frac{q^{\nu}}{(p+j)^{j}(q-p+\nu-j)^{\nu-j}} \geqslant \frac{q^{\nu}}{(p+\nu)^{j}(q-p+\nu)^{\nu-j}}=\left(\frac{q}{\max \{p+\nu, q-p+\nu\}}\right)^{\nu} .
\end{gathered}
$$

Тем самым, получена нижняя оценка в (9.11). Случай $0<j<\nu$ рассмотрен. Если в исходном условии $0 \leqslant j \leqslant \nu$ присутствует равенство, то некоторые сомножители в (9.12) будут не нужны, выкладки упростятся, а результат останется прежним. Лемма доказана.

Лемма 9.1 применима к коэффициентам $C_{q k+\nu}^{p k+j}$ со значениями $j=j_{p, q}(\nu)$ из формулы (3.3), так как заведомо $0 \leqslant j_{p, q}(\nu) \leqslant \nu$ (см. (3.5)). Теперь всё готово для доказательства результатов, сформулированных в разделе 8 .

10. Доказательства результатов о сходимости. Рассматриваем полиномы Бернштейна (1.1) от рационального модуля (1.2) при соглашениях (2.1).

Доказательство леммы 8.1. Оценим нужным образом разности $B_{q k+\nu+1}(f, z)-B_{q k+\nu}(f, z)$ при $k \in \mathbb{N}$ и $\nu=1, \ldots, q-1$. Случай $k=0$ обсудим потом отдельно.

Взяв за основу представление (6.2), выделим там агрегат

$$
\mathfrak{A}_{p, q}(z ; k, \nu) \equiv \frac{a_{p, q}(\nu)}{q k+\nu} C_{q k+\nu}^{p k+j_{p, q}(\nu)} z^{p k}(1-z)^{(q-p) k}
$$

со значениями $a_{p, q}(\nu), j_{p, q}(\nu)$, заданными правилами (3.3). Немного огрубив неравенства (3.5), имеем соотношения

$$
1 \leqslant a_{p, q}(\nu) \leqslant \max \{p, q-p\}=\frac{p(q-p)}{\min \{p, q-p\}}, \quad 0 \leqslant j_{p, q}(\nu) \leqslant \nu, \quad \nu=1, \ldots, q-1 .
$$

Заметим также, что

$$
z^{p k}(1-z)^{(q-p) k}=\left(z^{p}(1-z)^{q-p}\right)^{k}=\left(\frac{q^{q}}{p^{p}(q-p)^{q-p}}\right)^{-k}\left(T_{p, q}(z)\right)^{k}
$$

с первичным полиномом $T_{p, q}(z)$ из формулы $(8.9)$. Теперь запишем

$$
\left|\mathfrak{A}_{p, q}(z ; k, \nu)\right|=\frac{a_{p, q}(\nu)}{q} \cdot \frac{q k}{q k+\nu} \frac{C_{q k+\nu}^{p k+j_{p, q}(\nu)}}{C_{q k}^{p k}} \cdot\left(\frac{q^{q}}{p^{p}(q-p)^{q-p}}\right)^{-k} \frac{C_{q k}^{p k}}{k} \cdot\left|T_{p, q}(z)\right|^{k} .
$$

Принимая во внимание ограничения (10.2), а также учитывая оценки (9.10) и (9.11), получаем, что

$$
\left\{\begin{array}{l}
\left|\mathfrak{A}_{p, q}(z ; k, \nu)\right| \leqslant \frac{(q+\nu-1)^{\nu}}{(\min \{p, q-p\})^{\nu+1}} \sqrt{\frac{p(q-p)}{2 q \pi}} \frac{1}{k^{3 / 2}}\left|T_{p, q}(z)\right|^{k} \\
\left|\mathfrak{A}_{p, q}(z ; k, \nu)\right| \geqslant \frac{7}{8} \frac{q^{\nu-1}}{(\max \{p+\nu, q-p+\nu\})^{\nu}} \sqrt{\frac{q}{2 p(q-p) \pi}} \frac{1}{k^{3 / 2}}\left|T_{p, q}(z)\right|^{k}
\end{array}\right.
$$

при $z \in \mathbb{C}$ с полиномом $T_{p, q}(z)$ из формулы (8.9). Поясним на всякий случай, что при оценивании величины (10.3) мы приняли

$$
\frac{1}{q} \leqslant \frac{a_{p, q}(\nu)}{q} \leqslant \frac{p(q-p)}{q \min \{p, q-p\}}
$$

что допустимо в силу первого соотношения (10.2). Особо подчеркнем, что результат (10.4) получен при $k \in \mathbb{N}$ и $\nu=1, \ldots, q-1$. Случай $k=0$ по-прежнему пока не рассматриваем.

При помощи введенного обозначения (10.1) ключевую формулу (6.2) можно записать в виде

$$
B_{q k+\nu+1}(f, z)-B_{q k+\nu}(f, z)=-2 z^{\lceil p \nu / q\rceil}(1-z)^{\nu-\lfloor p \nu / q\rfloor} \mathfrak{A}_{p, q}(z ; k, \nu) .
$$


Пусть $k \in \mathbb{N}$ и $\nu=1, \ldots, q-1$. Оценим (10.6) по модулю отдельно для $z \in K_{p, q}$ и для $z \in \mathbb{C} \backslash K_{p, q}$ с компактом $K_{p, q}$ вида (8.4). Согласно классическим принципам максимума и минимума модуля (см. $[9$, с. 309]) замечаем, что

$$
\left\{\begin{aligned}
\max _{z \in K_{p, q}}\left|z^{\lceil p \nu / q\rceil}(1-z)^{\nu-\lfloor p \nu / q\rfloor}\right| & =\max _{z \in \Lambda_{p, q}}\left|z^{\lceil p \nu / q\rceil}(1-z)^{\nu-\lfloor p \nu / q\rfloor}\right|, \\
\inf _{z \notin K_{p, q}}\left|z^{\lceil p \nu / q\rceil}(1-z)^{\nu-\lfloor p \nu / q\rfloor}\right| & =\min _{z \in \Lambda_{p, q}}\left|z^{\lceil p \nu / q\rceil}(1-z)^{\nu-\lfloor p \nu / q\rfloor}\right|,
\end{aligned}\right.
$$

где $\Lambda_{p, q}$ - граница компакта $K_{p, q}$, т.е. лемниската Канторовича (8.3). Воспользуемся соотношениями (10.4) и (10.7) при оценке представления (10.6). Очевидными комбинациями указанных формул получаем два вывода:

(1) при $z \in K_{p, q}$ справедлива оценка сверху (8.11) с величиной $M_{p, q}(\nu)$ из формулы (8.7);

(2) при $z \in \mathbb{C} \backslash K_{p, q}$ справедлива оценка снизу (8.12) с величиной $L_{p, q}(\nu)$ из формулы (8.8).

Лемма 8.1 доказана.

Лемма 8.1 создает твердую основу для дальнейшего. Но прежде чем перейти к доказательству основных результатов «о сходимости» из теорем 8.1 и 8.2, полезно дополнить технический аппарат, связанный с разложениями Поповичу.

Напомним, что согласно установленной основной теореме 3.1 интересующие нас полиномы Бернштейна $B_{n}(f, z)$ от функции (1.2) можем выражать формулами (3.9), (3.10) через структурированные суммы (3.6).

Каждую такую сумму $\mathfrak{S}_{p, q}^{d}(z ; \nu)$ при фиксированном номере $\nu \in\{1, \ldots, q-1\}$ будем рассматривать как $d$-ю частичную сумму функционального ряда

$$
\mathfrak{S}_{p, q}(z ; \nu)=2 z^{\lceil p \nu / q\rceil}(1-z)^{\nu-\lfloor p \nu / q\rfloor} \sum_{k=0}^{\infty} \frac{a_{p, q}(\nu)}{q k+\nu} C_{q k+\nu}^{p k+j_{p, q}(\nu)}\left(z^{p}(1-z)^{q-p}\right)^{k} .
$$

Используя представление (6.2) из леммы 6.1, получаем эквивалентную запись

$$
\begin{aligned}
\mathfrak{S}_{p, q}(z ; \nu)=-\sum_{k=0}^{\infty}\left(B_{q k+\nu+1}(f, z)-B_{q k+\nu}(f, z)\right)= \\
=-\left(B_{\nu+1}(f, z)-B_{\nu}(f, z)\right)-\sum_{k=1}^{\infty}\left(B_{q k+\nu+1}(f, z)-B_{q k+\nu}(f, z)\right) .
\end{aligned}
$$

Лемма 8.1 устанавливает связь подобных рядов с понятиями «круга Канторовича». Имеются в виду лемниската $\Lambda_{p, q}$, компакт $K_{p, q}$ и первичный полином $T_{p, q}(z)$ из формул $(8.3),(8.4)$ и $(8.9)$ соответственно.

Действительно, пусть $z \in K_{p, q}$. Так как $\left|T_{p, q}(z)\right| \leqslant 1$ на компакте $K_{p, q}$ (см. (8.10)), то по лемме 8.1 мажорируем (10.9) следующим образом:

$$
\left|\mathfrak{S}_{p, q}(z ; \nu)\right| \leqslant M_{p, q}^{(0)}(\nu)+M_{p, q}(\nu) \sum_{k=1}^{\infty} \frac{1}{k^{3 / 2}}\left|T_{p, q}(z)\right|^{k} \leqslant M_{p, q}^{(0)}(\nu)+M_{p, q}(\nu) \sum_{k=1}^{\infty} \frac{1}{k^{3 / 2}}<+\infty .
$$

Здесь $M_{p, q}(\nu), M_{p, q}^{(0)}(\nu)$ суть конечные величины, первая - из формулы (8.7), а вторая - вида

$$
M_{p, q}^{(0)}(\nu) \equiv \max _{z \in K_{p, q}}\left|B_{\nu+1}(f, z)-B_{\nu}(f, z)\right|>0 .
$$

По признаку Вейерштрасса функциональный ряд (10.8) сходится абсолютно и равномерно на компакте $K_{p, q}$, где определена непрерывная сумма $\mathfrak{S}_{p, q}(z ; \nu)$. $\mathrm{K}$ ней сходятся частичные суммы $\mathfrak{S}_{p, q}^{d}(z ; \nu)$ при $d \rightarrow \infty$.

Внутренность компакта $K_{p, q}$ состоит из двух областей, ограниченных петлями лемнискаты $\Lambda_{p, q}$. Слагаемые ряда (10.8) - обычные полиномы, аналитические всюду в $\mathbb{C}$. По теореме Вейерштрасса о равномерно сходящихся рядах аналитических функций (см. [9, с. 265]) сумма $\mathfrak{S}_{p, q}(z ; \nu)$ будет раздельно аналитической внутри каждой открытой компоненты компакта $K_{p, q}$, т.е. внутри левой 
петли лемнискаты получится одна аналитическая предельная функция, а внутри правой петли другая, возможно, отличная от первой.

При $z \in \mathbb{C} \backslash K_{p, q}$ ряд (10.8) расходится. Для того чтобы это заметить, достаточно сравнить запись (10.9) с оценкой (8.12) из леммы 8.1. Поскольку $\left|T_{p, q}(z)\right|>1$ вне компакта $K_{p, q}$ (см. (8.10)), то из (8.12) следует, что во всякой точке $z \in \mathbb{C} \backslash K_{p, q}$ величина $\left|B_{q k+\nu+1}(f, z)-B_{q k+\nu}(f, z)\right|$ экспоненциально растет при $k \rightarrow \infty$ и уж никак не стремится к нулю. Тем самым, для (10.9) нарушено необходимое условие сходимости, что и влечет расходимость ряда (10.8). Для четкости изложения сформулируем установленный результат.

Теорема 10.1. Пусть $p, q$ - фиксированнные числа из (2.1). Тогда при любом заданном значении $\nu \in\{1, \ldots, q-1\}$ функциональный ряд (10.8) с величинами $a_{p, q}(\nu), j_{p, q}(\nu)$, определенными правилами (3.3), сходится абсолютно и равномерно на компакте $K_{p, q}$ из бормуль (8.4) и расходится в любой точке $z \in \mathbb{C} \backslash K_{p, q}$. Сумма рлда $\mathfrak{S}_{p, q}(z ; \nu)$ непрерывна на $K_{p, q}$ и раздельно аналитична внутри левой и правой петель этого компакта.

Теорема 10.1 полностью доказана. Устраним лишь один технический пробел, связанный с оценками агрегата $\mathfrak{A}_{p, q}(z ; k, \nu)$ при $k=0$. По очевидной причине (из-за деления на $k$ ) этот специфический случай выпал из общего результата (10.4).

Согласно определению (10.1) имеем выражение

$$
\mathfrak{A}_{p, q}(z ; 0, \nu)=\frac{a_{p, q}(\nu)}{\nu} C_{\nu}^{j_{p, q}(\nu)} \equiv \mathfrak{A}_{p, q}^{(0)}(\nu), \quad \nu=1, \ldots, q-1,
$$

по факту не зависящее от $z$. Для биномиальных коэффициентов возьмем «грубую» оценку

$$
1 \leqslant C_{\nu}^{j} \leqslant 2^{\nu} \sqrt{\frac{2}{\nu \pi}}, \quad j=0, \ldots, \nu
$$

Первая (нижняя) часть в (10.12) очевидна. Для доказательства оценки сверху рассмотрим отдельно четные $\nu=2 \mu$ и нечетные $\nu=2 \mu-1$. Используем неравенство Валлиса (9.4). При $\nu=2 \mu$ имеем

$$
\max _{0 \leqslant j \leqslant \nu} C_{\nu}^{j}=\max _{0 \leqslant j \leqslant 2 \mu} C_{2 \mu}^{j}=C_{2 \mu}^{\mu}<\frac{2^{2 \mu}}{\sqrt{\mu \pi}}=2^{\nu} \sqrt{\frac{2}{\nu \pi}} .
$$

При $\nu=2 \mu-1$ имеем

$$
\max _{0 \leqslant j \leqslant \nu} C_{\nu}^{j}=\max _{0 \leqslant j \leqslant 2 \mu-1} C_{2 \mu-1}^{j}=C_{2 \mu-1}^{\mu-1}=C_{2 \mu-1}^{\mu}=\frac{1}{2} C_{2 \mu}^{\mu}<\frac{2^{2 \mu-1}}{\sqrt{\mu \pi}}<2^{2 \mu-1} \sqrt{\frac{2}{(2 \mu-1) \pi}}=2^{\nu} \sqrt{\frac{2}{\nu \pi}} .
$$

Оценка (10.12) доказана.

Принимая во внимание оценку (10.12) и ограничения (10.2), получаем для агрегата (10.11), что

$$
\frac{1}{\nu} \leqslant \mathfrak{A}_{p, q}^{(0)}(\nu) \equiv \frac{a_{p, q}(\nu)}{\nu} C_{\nu}^{j_{p, q}(\nu)} \leqslant \max \{p, q-p\} \sqrt{\frac{2}{\pi}} \frac{2^{\nu}}{\nu^{3 / 2}}, \quad \nu=1, \ldots, q-1 .
$$

Соответственно, для величины $M_{p, q}^{(0)}(\nu)$ из формулы $(10.10)$, используя представление $(6.2)$, взятое при $k=0$, устанавливаем оценку сверху

$$
\begin{aligned}
& M_{p, q}^{(0)}(\nu) \equiv \max _{z \in K_{p, q}}\left|B_{\nu+1}(f, z)-B_{\nu}(f, z)\right|=2 \mathfrak{A}_{p, q}^{(0)}(\nu) \max _{z \in K_{p, q}}\left|z^{\lceil p \nu / q\rceil}(1-z)^{\nu-\lfloor p \nu / q\rfloor}\right| \leqslant \\
& \leqslant \max \{p, q-p\} \sqrt{\frac{2}{\pi}} \frac{2^{\nu+1}}{\nu^{3 / 2}} \max _{z \in \Lambda_{p, q}}\left|z^{\lceil p \nu / q\rceil}(1-z)^{\nu-\lfloor p \nu / q\rfloor}\right|, \quad \nu=1, \ldots, q-1 .
\end{aligned}
$$

Двойное неравенство (10.13) можно рассматривать как дополнение к прежней формуле (10.4); цепочка же (10.14) дает некий аналог оценки (8.11) в пропущенном ранее случае $k=0$. Эти результаты полезно иметь для полноты изложения.

Доказательство теоремы 8.1. Напомним, что надо установить два факта: во-первых, сходимость полиномов Бернштейна от рационального модуля (1.2) к соответствующей предельной функции (8.6) всюду на компакте $K_{p, q}$; а, во-вторых, расходимость тех же полиномов всюду вне 
компакта $K_{p, q}$. По-прежнему считаем, что компакт Канторовича $K_{p, q}$ определен формулой (8.4) для фиксированной порождающей функции $f(x)=|q x-p|$, взятой при соглашениях $(2.1)$.

Начнем со сходимости. Она теперь доказывается несложно. По теореме 3.1 изучаемые полиномы $B_{n}(f, z)$ при $n \geqslant q$ представимы формулами (3.9) и (3.10), составленными из $q-1$ структурированных сумм вида (3.6). Каждая такая сумма при фиксированном $\nu \in\{1, \ldots, q-1\}$ есть частичная сумма соответствующего функционального ряда (10.8). По теореме 10.1 все эти ряды (в конечном числе $q-1$ ) равномерно сходятся на одном и том же компакте Канторовича $K_{p, q}$. Представления (3.9) и (3.10) показывают, что на том же компакте при любом фиксированном значении $r \in\{0, \ldots, q-1\}$ будет сходиться подпоследовательность полиномов

$$
B_{q m+r}(f, z), \quad m=1,2,3, \ldots,
$$

имея в качестве предельной функции комбинацию

$$
p+(q-2 p) z-\sum_{\nu=1}^{q-1} \mathfrak{S}_{p, q}(z ; \nu), \quad z \in K_{p, q},
$$

составленную из сумм функциональных рядов (10.8).

Очевидно, результат (10.16) не зависит от выбора значения $r \in\{0, \ldots, q-1\}$, т.е. не зависит от взятой подпоследовательности (10.15). Совокупность таких подпоследовательностей образует в итоге всю последовательность $B_{n}(f, z)$, начиная с номера $n=q$. Следовательно, при $n \rightarrow \infty$ полиномы $B_{n}(f, z)$ также равномерно сходятся на компакте Канторовича $K_{p, q}$ и имеют в качестве предела функцию (10.16).

В силу теоремы 10.1 эта предельная функция (10.16) должна быть раздельно аналитической внутри левой и правой петель компакта $K_{p, q}$. Но в левой петле на отрезке $[0, p / q] \subset \mathbb{R}$, согласно изначальной теореме Бернштейна, последовательность $B_{n}(f, x)$ при $n \rightarrow \infty$ сходится к значениям $f(x)=p-q x$, а в правой петле на отрезке $[p / q, 1] \subset \mathbb{R}$ последовательность $B_{n}(f, x)$ при $n \rightarrow \infty$ сходится к значениям $f(x)=q x-p$. Для данных значений единственным кусочно аналитическим продолжением в две замкнутые области (8.5) компакта $K_{p, q}$ служит функция $\varphi(z)$ из формулы (8.6). Тем самым, приходим к равенству

$$
p+(q-2 p) z-\sum_{\nu=1}^{q-1} \mathfrak{S}_{p, q}(z ; \nu)=\varphi(z) \quad\left(\equiv\left\{\begin{array}{ll}
p-q z, & z \in K_{p, q}^{(1)}, \\
q z-p, & z \in K_{p, q}^{(2)}
\end{array}\right),\right.
$$

дающему окончательное описание предельной функции полиномов Бернштейна $B_{n}(f, z)$. Это и утверждалось в теореме 8.1.

Осталось показать расходимость полиномов $B_{n}(f, z)$ всюду вне компакта $K_{p, q}$. При этом мы не можем использовать расходимость на $\mathbb{C} \backslash K_{p, q}$ структурированных сумм (3.6), вытекающую из теоремы 10.1, поскольку из расходимости таких слагаемых еще не следует расходимость общих выражений (3.9) и (3.10). (Как известно, при сложении нескольких расходящихся рядов может образоваться сходящийся ряд, частичные суммы которого имеют конечный предел.)

Поступим проще. Допустим, что имеется точка $z_{0} \in \mathbb{C} \backslash K_{p, q}$, в которой существует конечный предел

Тогда

$$
\lim _{n \rightarrow \infty} B_{n}\left(f, z_{0}\right)=A \in \mathbb{C}
$$

Последнее означает, что $\left|B_{n+1}\left(f, z_{0}\right)-B_{n}\left(f, z_{0}\right)\right| \rightarrow 0$ при $n \rightarrow \infty$. Но это невозможно в силу оценки (8.12) из леммы 8.1, где $\left|T_{p, q}(z)\right|>1$ при любом $z \in \mathbb{C} \backslash K_{p, q}$ (см. (8.10)). Итак, предположение о существовании конечного предела (10.18) в некоторой внешней точке $z_{0} \in \mathbb{C} \backslash K_{p, q}$ приводит к противоречию с доказанной оценкой (8.12). Следовательно, во всех точках $z \in \mathbb{C} \backslash K_{p, q}$ последовательность $B_{n}(f, z)$ расходится. Теорема 8.1 полностью доказана.

Доказательство теоремы 8.2. Пусть снова $B_{n}(f, z)$ - полиномы Бернштейна (1.1) от функции (1.2) при соглашениях (2.1). Как прежде, через $K_{p, q}$ обозначаем компакт Канторовича (8.4). Согласно (8.14) определим уклонения $R_{n}(f, z)$ полиномов $B_{n}(f, z)$ от предельной функции $\varphi(z)$ 
вида (8.6). Но функция $\varphi(z)$ по формуле (10.17) выражается через функциональные ряды (10.8). Иначе говоря, справедливо представление

$$
\varphi(z)=p+(q-2 p) z-\sum_{\nu=1}^{q-1} 2 z^{\lceil p \nu / q\rceil}(1-z)^{\nu-\lfloor p \nu / q\rfloor} \sum_{k=0}^{\infty} \frac{a_{p, q}(\nu)}{q k+\nu} C_{q k+\nu}^{p k+j_{p, q}(\nu)}\left(z^{p}(1-z)^{q-p}\right)^{k}
$$

при $z \in K_{p, q}$ с величинами $a_{p, q}(\nu), j_{p, q}(\nu)$ из формулы (3.3). Ориентируясь на конструкцию (3.1), рассмотрим уклонения

$$
R_{q m+r}(f, z)=B_{q m+r}(f, z)-\varphi(z), \quad z \in K_{p, q}
$$

при $m \in \mathbb{N}, r=0,1, \ldots, q-1$. Взяв разность представлений (3.1) и (10.19), получим выражение

$$
R_{q m+r}(f, z)=\sum_{\nu=1}^{q-1} 2 z^{\lceil p \nu / q\rceil}(1-z)^{\nu-\lfloor p \nu / q\rfloor} \sum_{k=\varepsilon_{m}(\nu, r)+1}^{\infty} \frac{a_{p, q}(\nu)}{q k+\nu} C_{q k+\nu}^{p k+j_{p, q}(\nu)}\left(z^{p}(1-z)^{q-p}\right)^{k}, \quad z \in K_{p, q},
$$

где символ $\varepsilon_{m}(\nu, r)$ вводится по правилу (3.2).

Вспоминая про связь (6.2), запишем через разности

$$
R_{q m+r}(f, z)=-\sum_{\nu=1}^{q-1} \sum_{k=\varepsilon_{m}(\nu, r)+1}^{\infty}\left(B_{q k+\nu+1}(f, z)-B_{q k+\nu}(f, z)\right), \quad z \in K_{p, q}
$$

при $m \in \mathbb{N}, r=0,1, \ldots, q-1$. Соотношение (10.20) и будем оценивать.

Посредством базовой формулы (8.11) из леммы 8.1 получаем

$$
\left|R_{q m+r}(f, z)\right| \leqslant \sum_{\nu=1}^{q-1} M_{p, q}(\nu) \sum_{k=\varepsilon_{m}(\nu, r)+1}^{\infty} \frac{1}{k^{3 / 2}}\left|T_{p, q}(z)\right|^{k}, \quad z \in K_{p, q}
$$

с коэффициентами $M_{p, q}(\nu)$ вида $(8.7)$ и величиной $\left|T_{p, q}(z)\right| \leqslant 1$, записанной через первичный полином Канторовича (8.9) (см. также (8.10)). Поскольку $m \leqslant \varepsilon_{m}(\nu, r)+1 \leqslant m+1$ (см. (3.2)), то

$$
\left|R_{q m+r}(f, z)\right| \leqslant \sum_{\nu=1}^{q-1} M_{p, q}(\nu) \sum_{k=m}^{\infty} \frac{1}{k^{3 / 2}}\left|T_{p, q}(z)\right|^{k}, \quad z \in K_{p, q}
$$

Короче,

$$
\left|R_{q m+r}(f, z)\right| \leqslant M_{p, q} \sum_{k=m}^{\infty} \frac{1}{k^{3 / 2}}\left|T_{p, q}(z)\right|^{k}, \quad z \in K_{p, q}
$$

при $m \in \mathbb{N}, r=0,1, \ldots, q-1$, с суммарной величиной $M_{p, q}$ вида (8.13).

Оценка (10.21) является ключевой. Она вполне достаточна для получения нужных результатов. Дальнейшие рассуждения зависят от того, попадает точка $z$ внутрь компакта $K_{p, q}$ или находится на его границе.

Пусть $z \in \operatorname{int} K_{p, q}$. Тогда $\left|T_{p, q}(z)\right|<1$ (см. (8.10)), и формула (10.21) дает оценку

$$
\left|R_{q m+r}(f, z)\right| \leqslant M_{p, q} \frac{1}{m^{3 / 2}} \sum_{k=m}^{\infty}\left|T_{p, q}(z)\right|^{k}=\frac{M_{p, q}}{1-\left|T_{p, q}(z)\right|} \frac{\left|T_{p, q}(z)\right|^{m}}{m^{3 / 2}}, \quad m \in \mathbb{N}, \quad r=0,1, \ldots, q-1,
$$

что совпадает с заявленным соотношением (8.15).

Пусть $z \in \partial K_{p, q}=\Lambda_{p, q}$. Тогда $\left|T_{p, q}(z)\right|=1$ (см. снова (8.10)), и формула (10.21) дает оценку

$$
\left|R_{q m+r}(f, z)\right| \leqslant M_{p, q} \sum_{k=m}^{\infty} \frac{1}{k^{3 / 2}}, \quad m \in \mathbb{N}, \quad r=0,1, \ldots, q-1 .
$$

Ho

$$
\sum_{k=m}^{\infty} \frac{1}{k^{3 / 2}}=\frac{1}{m^{3 / 2}}+\sum_{k=m+1}^{\infty} \frac{1}{k^{3 / 2}} \leqslant \frac{1}{m^{3 / 2}}+\int_{m}^{\infty} \frac{d x}{x^{3 / 2}}=\frac{1}{m \sqrt{m}}+\frac{2}{\sqrt{m}}=\frac{2}{\sqrt{m}}\left(1+\frac{1}{2 m}\right)
$$

при любом $m \in \mathbb{N}$. В итоге получаем нужное соотношение (8.16). Теорема 8.2 доказана. 
Как видим, заключительный этап доказательства идейно прост: после того, как всю конструкцию удалось собрать в краткую формулу (10.21), использовались лишь самые элементарные приемы, связанные с геометрической прогрессией и оценкой (10.22) типа «Коши-Маклорена».

При этом установленные результаты качественно вполне точны. Из теоремы 8.2 видно главное: внутри компакта $K_{p, q}$ изучаемые полиномы $B_{n}(f, z)$ сходятся к предельной функции с экспоненциальной скоростью, а на границе компакта действует другой, более медленный степенной закон. Как показывает наработанный нами опыт (см., например, [21] или $[22$, разд. 12, 13]), оценки $(8.15)$ и (8.16) правильно отражают суть дела. Их некоторый недостаток связан со взаимной несогласованностью в том смысле, что «граничную» оценку (8.16) нельзя вывести из «внутренней» оценки (8.15) из-за «разрушения» (8.15) при подстановке туда предельного значения $\left|T_{p, q}(z)\right|=1$.

Покажем, что при помощи более тонких методов можно избежать подобной проблемы и дать совмещенную оценку уклонения $R_{n}(f, z)$, пригодную как внутри компакта $K_{p, q}$, так и на его границе. При этом внутри $K_{p, q}$ новая оценка будет даже чуть лучше, чем прежняя оценка (8.15).

11. Совмещенная оценка уклонения. Некоторые оценки такого рода были анонсированы в обзоре [22] для полиномов Бернштейна от симметричного модуля $f(x)=|2 x-1|$. Эти результаты касались сходимости полиномов лишь на $[0,1]$, но охватывали как саму точку излома $x=1 / 2$ (где уклонение стремится к нулю степенным образом), так и все прочие точки $x \in[0,1 / 2) \cup(1 / 2,1]$ (где действует экспоненциальный закон стремления к нулю). Использованный метод был связан с каноническим разложением Поповичу (3.13).

Через некоторое время А. Ю. Попов заметил (см. [17]), что рассуждения существенно упрощаются, а результат становится точнее, если предварительно установить специальную оценку остатков степенного ряда с положительными логарифмически выпуклыми коэффициентами. Идея Попова оказалась весьма удачной. Сформулируем его результат.

Рассмотрим последовательность числовых коэффициентов $\left(a_{k}\right)_{k=1}^{\infty}$, обладающую следующими свойствами:

$$
a_{k}>0 \quad \forall k \in \mathbb{N} ; \quad \sum_{k=1}^{\infty} a_{k}<+\infty .
$$

Составим степенной ряд

$$
\sum_{k=1}^{\infty} a_{k} s^{k}<+\infty
$$

вещественной переменной $s \in[0,1]$ и возьмем остатки

$$
h_{m} \equiv \sum_{k=m}^{\infty} a_{k}, \quad H_{m}(s) \equiv \sum_{k=m}^{\infty} a_{k} s^{k}, \quad m \in \mathbb{N} .
$$

Тогда для любого $m \in \mathbb{N}$ верны соотношения:

(a) $H_{m}(s)$ строго возрастает на $[0,1]$;

(b) $0=H_{m}(0)<H_{m}(s)<H_{m}(1)=h_{m}<+\infty$ при $s \in(0,1)$;

(c) $H_{m}(s) \sim a_{m} s^{m}$ при $s \rightarrow 0+$.

Ставится вопрос о получении возможно более точных оценок сверху для $H_{m}(s)$ посредством простых, эталонных величин $F_{m}(s)$, удовлетворяющих тем же соотношениям (a)-(c) при замене в них $H_{m}(s)$ на $F_{m}(s)$.

Оказывается, эта задача допускает явное решение при дополнительном условии логарифмической выпуклости коэффициентов:

$$
a_{k+1}^{2} \leqslant a_{k} a_{k+2}, \quad k \in \mathbb{N} .
$$

Справедлив следующий результат (А. Ю. Попов).

Лемма 11.1. Пусть коэффициенты степенного ряда (11.2) удовлетворяют условиям (11.1) u (11.4). Пусть остатки $h_{m}$ и $H_{m}(s)$ определены формулой (11.3). Тогда

$$
H_{m}(s) \leqslant F_{m}(s) \equiv \frac{h_{m}}{h_{m}-h_{m+1} s} a_{m} s^{m}, \quad s \in[0,1], \quad m \in \mathbb{N} .
$$


Функиия $F_{m}(s)$ из формулы (11.5) удовлетворяет указанным выше условиям (а)-(с) при замене в них $H_{m}(s)$ на $F_{m}(s)$.

Доказательство. Данное утверждение фактически установлено в [17, теорема 1]. Следует лишь иметь в виду, что оценка, эквивалентная (11.5), получена в [17] в других обозначениях для нормированных остатков

$$
H_{m}^{(0)}(s) \equiv \sum_{k=m}^{\infty} a_{k} s^{k-m}, \quad H_{m}(s)=s^{m} H_{m}^{(0)}(s), \quad s \in[0,1], \quad m \in \mathbb{N} .
$$

Сведение результата [17] к виду (11.5) элементарно. То, что все функции $F_{m}(s)$ из формулы $(11.5)$ удовлетворяют указанным выше условиям (a)-(c), проверяется непосредственно.

Сохранение условий (a)-(c) для элементарных функций $F_{m}(s)$ указывает на высокую точность оценки (11.5). Однако на практике применение (11.5) затрудняется тем, что числовые остатки $h_{m}$ не всегда вычисляются явно. Вместо их точных значений, чаще удается установить неравенства

$$
h_{m} \equiv \sum_{k=m}^{\infty} a_{k} \leqslant\left(1+\gamma_{m}\right) a_{m}, \quad m \in \mathbb{N},
$$

с конструктивно вычисляемыми поправками $\gamma_{m}>0$. В таком случае справедлив следующий «огрубленный» вариант леммы 11.1.

Лемма 11.2. Пусть коэффициенты степенного рлда (11.2) удовлетворяют условиям (11.1) u (11.4). Пусть остатки $h_{m}$ и $H_{m}(s)$ определены формулой (11.3), причем для $h_{m}$ справедливы оченки (11.6) со значениями $\gamma_{m}>0$. Тогда

$$
H_{m}(s) \leqslant \Phi_{m}(s) \equiv \frac{1+\gamma_{m}}{1+\gamma_{m}-\gamma_{m} s} a_{m} s^{m}, \quad s \in[0,1], \quad m \in \mathbb{N} .
$$

Доказательство. Из (11.6) следует, что

$$
\frac{h_{m+1}}{h_{m}}=\frac{h_{m}-a_{m}}{h_{m}}=1-\frac{a_{m}}{h_{m}} \leqslant 1-\frac{1}{1+\gamma_{m}}=\frac{\gamma_{m}}{1+\gamma_{m}}, \quad m \in \mathbb{N} .
$$

Соответственно,

$$
\frac{h_{m}-h_{m+1} s}{h_{m}}=1-\frac{h_{m+1}}{h_{m}} s \geqslant 1-\frac{\gamma_{m}}{1+\gamma_{m}} s=\frac{1+\gamma_{m}-\gamma_{m} s}{1+\gamma_{m}}, \quad s \in[0,1], \quad m \in \mathbb{N} .
$$

Тем самым, мажоранты в оценке (11.5) сами мажорируются так:

$$
F_{m}(s) \equiv \frac{h_{m}}{h_{m}-h_{m+1} s} a_{m} s^{m} \leqslant \frac{1+\gamma_{m}}{1+\gamma_{m}-\gamma_{m} s} a_{m} s^{m} \equiv \Phi_{m}(s), \quad s \in[0,1], \quad m \in \mathbb{N} .
$$

Используя это соображение в (11.5), получаем нужный результат (11.7). Лемма доказана.

Как видно из формулы (11.8), обе мажоранты-и более точная $F_{m}(s)$, и более «грубая» $\Phi_{m}(s)$ - имеют одинаковую структуру. Фактически, всё их различие заключено в краевых условиях

$$
F_{m}(1)=h_{m} \leqslant \Phi_{m}(1)=\left(1+\gamma_{m}\right) a_{m}, \quad m \in \mathbb{N},
$$

причем условие для $\Phi_{m}(s)$ «завышено» ровно настолько, насколько неточны были первоначальные оценки (11.6). Так как $h_{m}=a_{m}+h_{m+1}$, то для получения «хороших» результатов в (11.6) достаточно при любом $m \in \mathbb{N}$ правильно оценить остаток $h_{m+1}$ через предыдущий коэффициент $a_{m}$. На практике это часто удается сделать.

Разберем модельный пример. Рассмотрим степенной ряд

$$
\sum_{k=1}^{\infty} \frac{s^{k}}{k^{\alpha}}, \quad s \in[0,1]
$$


с параметром $\alpha>1$. Коэффициенты $a_{k}=1 / k^{\alpha}$ удовлетворяют условиям (11.1) и (11.4). При этом

$$
h_{m+1} \equiv \sum_{k=m+1}^{\infty} \frac{1}{k^{\alpha}} \leqslant \int_{m}^{+\infty} \frac{d x}{x^{\alpha}}=\frac{1}{(\alpha-1) m^{\alpha-1}}=\frac{m}{\alpha-1} a_{m}, \quad m \in \mathbb{N} .
$$

Тем самым, для оценок (11.6) найдены значения $\gamma_{m}=m /(\alpha-1)$. Применяя лемму 11.2, устанавливаем следующий результат.

Лемма 11.3. При любом фиксированном $\alpha>1$ справедливы оченки

$$
H_{m}(s ; \alpha) \equiv \sum_{k=m}^{\infty} \frac{s^{k}}{k^{\alpha}} \leqslant \frac{m+\alpha-1}{m+\alpha-1-m s} \cdot \frac{s^{m}}{m^{\alpha}}, \quad s \in[0,1], \quad m \in \mathbb{N} .
$$

Доказательство. Указанные оценки (11.10) получаются простой подстановкой в (11.7) коэффициентов $a_{m}=1 / m^{\alpha}$ и найденных выше значений $\gamma_{m}=m /(\alpha-1)$. Лемма доказана.

С прицелом на базовое соотношение (10.21), оценивающее уклонения полиномов Бернштейна, возьмем значение $\alpha=3 / 2$. Формула (11.10) показывает, что тогда

$$
\sum_{k=m}^{\infty} \frac{s^{k}}{k^{3 / 2}} \leqslant \frac{2 m+1}{2 m+1-2 m s} \cdot \frac{s^{m}}{m^{3 / 2}}=\frac{2 m+1}{2 m(1-s)+1} \cdot \frac{s^{m}}{m^{3 / 2}}, \quad s \in[0,1], \quad m \in \mathbb{N} .
$$

Теперь всё готово для доказательства следующего основного результата.

Теорема 11.1. Пусть $B_{n}(f, z)$ - полиномы Бернштейна (1.1) от функиии (1.2) при соглашениях (2.1). Пусть $R_{n}(f, z)$ - уклонения этих полиномов от предельной функиии (8.6), заданные по формуле (8.14). Тогда при $z \in K_{p, q}$, т.е. всюду на компакте Канторовича (8.4), имеем оченку

$$
\left|R_{q m+r}(f, z)\right| \leqslant \frac{(2 m+1) M_{p, q}}{2 m\left(1-\left|T_{p, q}(z)\right|\right)+1} \frac{\left|T_{p, q}(z)\right|^{m}}{m^{3 / 2}}, \quad m \in \mathbb{N}, \quad r=0,1, \ldots, q-1,
$$

с величинами $T_{p, q}(z), M_{p, q}$ из бормул (8.9) и (8.13) соответственно. При $z \in \partial K_{p, q}=\Lambda_{p, q}$ новая оценка (11.12) совпадает с прежней оценкой (8.16), а при $z \in \operatorname{int} K_{p, q}$ оценка (11.12) является более точной, чем прежняя оценка (8.15).

Доказательство. Как установлено в разделе 10 при доказательстве теоремы 8.2, для изучаемых уклонений $R_{q m+r}(f, z)$ справедлива оценка $(10.21)$, содержащая в своем составе $m$-й остаток ряда вида (11.9) с параметром $\alpha=3 / 2$ и переменной $s=\left|T_{p, q}(z)\right| \in[0,1]$ (см. также (8.10)). Оценим этот остаток по правилу (11.11) и получим из (10.21) нужное соотношение (11.12). Для того чтобы сравнить новый результат с прежними оценками, рассмотрим отдельно правую часть в (11.12).

Заметим, что

$$
\frac{(2 m+1) M_{p, q}}{2 m\left(1-\left|T_{p, q}(z)\right|\right)+1} \frac{\left|T_{p, q}(z)\right|^{m}}{m^{3 / 2}}=\frac{2 M_{p, q}}{2 m\left(1-\left|T_{p, q}(z)\right|\right)+1}\left(1+\frac{1}{2 m}\right) \frac{\left|T_{p, q}(z)\right|^{m}}{\sqrt{m}} .
$$

Отсюда при $z \in \partial K_{p, q}=\Lambda_{p, q}$, т.е. когда $\left|T_{p, q}(z)\right|=1$, имеем прежнюю оценку (8.16). С другой стороны, при $z \in \operatorname{int} K_{p, q}$, т.е. когда $\left|T_{p, q}(z)\right|<1$, получаем

$$
\frac{(2 m+1) M_{p, q}}{2 m\left(1-\left|T_{p, q}(z)\right|\right)+1} \frac{\left|T_{p, q}(z)\right|^{m}}{m^{3 / 2}}=\frac{M_{p, q}}{1-\frac{2 m}{2 m+1}\left|T_{p, q}(z)\right|} \frac{\left|T_{p, q}(z)\right|^{m}}{m^{3 / 2}}<\frac{M_{p, q}}{1-\left|T_{p, q}(z)\right|} \frac{\left|T_{p, q}(z)\right|^{m}}{m^{3 / 2}} .
$$

Тем самым, оценка (11.12) точнее прежнего результата (8.15). Теорема доказана.

Используя более изощрённые методы, можно, что называется, слегка «подтянуть» результат, заложенный в формуле (11.11). Это даст дополнительное улучшение оценки (11.12). Однако тогда при небольшом увеличении точности мы сильно ухудшим запись и получим более громоздкое выражение, не слишком информативное и трудное для восприятия. Ситуация хорошо выражается известной поговоркой «игра не стоит свеч». Неформально говоря, основное математическое содержание из базового соотношения (10.21) уже «выжато». Представляется, что дальнейшие 
уточнения оценок уклонения полиномов Бернштейна от рационального модуля лучше проводить в двух других направлениях.

Во-первых, путем специальной работы с величинами $\left|R_{q m+r}(f, z)\right|$ можно учесть влияние на результат не только основного переменного параметра $m \in \mathbb{N}$, но и остатка $r \in\{0,1, \ldots, q-1\}$. Этот технический анализ был бы полезен для завершения картины.

Во-вторых, и это, по-видимому, более интересно, следует уточнить значение коэффициента $M_{p, q}$ в оценке (11.12). При нашем подходе величина $M_{p, q}$ находится по формуле (8.13) - как сумма технических констант $M_{p, q}(\nu)$, вычисляемых по правилу (8.7). Было бы любопытно проанализировать все сделанные нами загрубления и поставить вопрос об экстремально точном значении коэффициента $M_{p, q}$, при котором результат вида (11.12) останется справедливым.

Ряд возможных улучшений нетрудно увидеть. Так, при оценке разностей (10.6) можно не проводить загрублений, связанных с переходами (10.5) и (10.7), а сохранить в мажорирующем коэффициенте значение $a_{p, q}(\nu)$, заданное по правилу (3.3), и допустить переменную $z \in K_{p, q}$.

Точнее, вместо величины (8.7) введем модифицированное выражение

$$
M_{p, q}^{*}(z ; \nu) \equiv a_{p, q}(\nu)\left(\frac{q+\nu-1}{\min \{p, q-p\}}\right)^{\nu} \sqrt{\frac{2}{p q(q-p) \pi}}\left|z^{\lceil p \nu / q\rceil}(1-z)^{\nu-\lfloor p \nu / q\rfloor}\right|,
$$

где $\nu=1, \ldots, q-1$. Затем заменим суммарную величину $M_{p, q}$ из формулы (8.13) ее прямым аналогом

$$
M_{p, q}^{*}(z) \equiv \sum_{\nu=1}^{q-1} M_{p, q}^{*}(z ; \nu), \quad z \in K_{p, q}
$$

Несложный анализ наших рассуждений показывает, что основная оценка (10.21) останется верной со значением (11.14) вместо прежнего $M_{p, q}$. Соответственно, в оценке уклонения (11.12) можно заменить коэффициент $M_{p, q}$ значением $M_{p, q}^{*}(z)$, вычисленным по правилам (11.13) и (11.14). Результат теоремы 11.1 станет точнее, ибо $M_{p, q}^{*}(z)<M_{p, q}$ при $z \in \operatorname{int} K_{p, q}$.

Рассмотрим пример, когда величина $M_{p, q}^{*}(z)$ вычисляется сразу, и оценка (11.12) допускает наглядную запись без каких-либо скрытых деталей.

Пусть $f(x)=|2 x-1|$ на $[0,1]$. Здесь $p=1, q=2$. По формуле (8.9) первичный полином Канторовича принимает вид $T_{1,2}(z)=4 z(1-z)$, порождая соответствующие компакт и лемнискату

$$
K_{1,2}: 4|z(1-z)| \leqslant 1, \quad \Lambda_{1,2}: 4|z(1-z)|=1 .
$$

Ограничение на параметр $\nu \in\{1, \ldots, q-1\}$ дает единственное значение $\nu=1$. Применяя формулы (11.14) и (11.13) с учетом равенства $a_{1,2}(1)=1$ (см. пример 7.1), находим, что

$$
M_{1,2}^{*}(z)=M_{1,2}^{*}(z ; 1)=\frac{2}{\sqrt{\pi}}|z(1-z)|=\frac{1}{2 \sqrt{\pi}} \cdot 4|z(1-z)| .
$$

Воспользуемся оценкой $(11.12)$, заменив в ней коэффициент $M_{1,2}\left(=M_{p, q}\right)$ на величину $M_{1,2}^{*}(z)$ из формулы (11.15). При $4|z(1-z)| \leqslant 1$ получим

$$
\left|R_{2 m}(f, z)\right| \leqslant \frac{1}{2 \sqrt{\pi}} \frac{2 m+1}{2 m(1-4|z(1-z)|)+1} \frac{(4|z(1-z)|)^{m+1}}{m^{3 / 2}}, \quad m \in \mathbb{N} .
$$

Величины $\left|R_{2 m+1}(f, z)\right|$ сейчас не рассматриваем, так как они совпадают с $\left|R_{2 m}(f, z)\right|$ из-за правила склеивания $B_{2 m+1}(f, z)=B_{2 m}(f, z)$ (см. лемму 4.1).

При $z=x \in[0,1]$ полиномы Бернштейна сходятся к функции $f(x)=|2 x-1|$ монотонно сверху (см. (4.6)), и все уклонения $R_{2 m}(f, x)$ будут неотрицательны. Кроме того, возможен переход

$$
1-4|x(1-x)|=1-4 x(1-x)=(2 x-1)^{2}, \quad x \in[0,1] .
$$

Проведя элементарные преобразования в (11.16), получим оценку

$$
0 \leqslant R_{2 m}(f, x) \leqslant \frac{1}{\sqrt{m \pi}}\left(1+\frac{1}{2 m}\right) \frac{(4 x(1-x))^{m+1}}{2 m(2 x-1)^{2}+1}, \quad x \in[0,1], \quad m \in \mathbb{N} .
$$


Последняя согласована с асимптотикой

$$
R_{2 m}(f, x) \sim \frac{1}{\sqrt{m \pi}} \frac{(4 x(1-x))^{m+1}}{2 m(2 x-1)^{2}+1}, \quad m \rightarrow \infty,
$$

верной при любом фиксированном $x \in(0,1)$ (см. обзор [22, формулы (52), (53), (65)]).

Оценка (11.17), извлеченная сейчас из наших общих результатов, лишь немногим хуже прежней, наиболее точной оценки

$$
0 \leqslant R_{2 m}(f, x) \leqslant 2^{-2 m} C_{2 m}^{m} \frac{(4 x(1-x))^{m+1}}{(2 m+1)(2 x-1)^{2}+1}, \quad x \in[0,1], \quad m \in \mathbb{N},
$$

полученной А. Ю. Поповым в [17] на основе его «незагрубленного» результата типа леммы 11.1.

Вообще, вопрос о сходимости полиномов Бернштейна от симметричного модуля может быть изучен с исчерпывающей полнотой. По этой тематике запланирован отдельный обзор [21]. Там, в частности, будет показано, что точная оценка (11.18) допускает простое доказательство, причем самым элементарным методом.

12. Проблема нулей. Итак, обобщенные разложения Поповичу связывают теорию полиномов Бернштейна от рациональных модулей вида (1.2) с классической теорией степенных рядов. Аналогия со степенными рядами полезна и при изучении вопроса о распределении нулей полиномов Бернштейна.

Напомним, что согласно классической теореме Р. Иентча (см. [45, с. 227]) для степенного ряда

$$
S(\zeta)=\sum_{k=0}^{\infty} \alpha_{k} \zeta^{k}
$$

переменной $\zeta \in \mathbb{C}$ с коэффициентами $\alpha_{k} \in \mathbb{C}$ и конечным радиусом сходимости $R>0$ граница круга сходимости $|\zeta|=R$ состоит из предельных точек множества нулей, взятых от частичных сумм этого ряда (см. также [10, с. 165-166], [18, с. 246-249]). Попросту говоря, значительная часть нулей от частичных сумм

$$
S_{n}(\zeta)=\sum_{k=0}^{n} \alpha_{k} \zeta^{k}, \quad \zeta \in \mathbb{C},
$$

при $n \rightarrow \infty$ стягивается к окружности $|\zeta|=R$, заполняя в пределе всю окружность. Более того, при некоторых условиях регулярности, наложенных на коэффициенты $\alpha_{k}$, можно утверждать, что в определенном смысле «почти все» нули частичных сумм (12.2) при $n \rightarrow \infty$ устремляются к окружности $|\zeta|=R$ (см. [44]). Дополнительную информацию, связанную с теоремой Иентча, см. в [48, с. 90-95, 145-148] и [54, с. 390-392, 396-399] (см. также [40,43]).

Некоторое время назад И. Я. Новиков на одном частном примере показал (см. [12]), что похожий принцип «концентрации нулей» характерен и для теории полиномов Бернштейна. В последующем цикле работ (см. [25-28,32]) авторы настоящего обзора провели систематическое компьютерное исследование проблемы нулей и выдвинули концепцию аттракторов нулей для классических полиномов Бернштейна.

Поясним, что аттрактором нулей мы называем предельное множество, к которому стягиваются все (или почти все) нули полиномов Бернштейна при выборе той или иной порождающей функции $f \in C[0,1]$. В $[26,27]$ представлен полный список правил для построения аттракторов нулей в случае произвольных кусочно линейных порождающих функций с рациональными абсциссами точек излома. Многочисленные примеры и иллюстрации по теме см. в [32].

Обычно значимую часть аттрактора составляет граница комплексной области сходимости полиномов Бернштейна. При наличии у порождающей функции нескольких точек излома в аттрактор включаются дополнительные артефакты - дуги специальных «серединных» окружностей. Впрочем, при выборе интересующего нас сейчас модельного примера рационального модуля (1.2) структура аттрактора может быть описана очень просто. Компьютерные эксперименты показывают, что справедливо следующее качественное утверждение. 


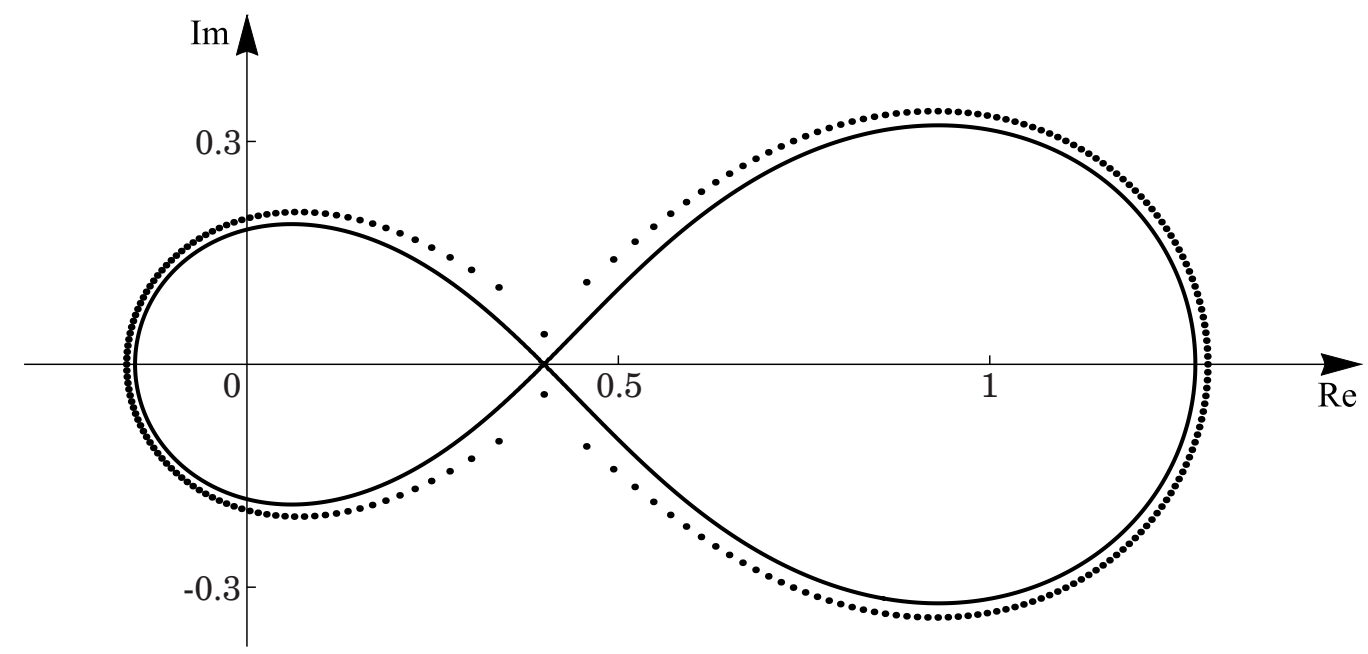

Рис. 2. Множество нулей полинома $B_{250}(f, z)$ для функции $f(x)=|5 x-2|$ вместе с соответствующим аттрактором нулей-лемнискатой Канторовича $\Lambda_{2,5}$, построенной по формуле (8.3) при $p=2, q=5$. Полином взят из цепочки склеиваний. Девиантные нули отсутствуют.

Принцип концентрации нулей. Пусть $B_{n}(f, z)$ - полиномь Бернштейна (1.1) от функции (1.2) при соглашениях (2.1). Тогда при $n \rightarrow \infty$ все нули полиномов $B_{n}(f, z)$ (за исключением отдельных «девиантных» нулей) стягиваются снаружи к границе области сходимости полиномов Бернштейна - лемнискате Канторовича $\Lambda_{p, q}$ из формулы (8.3). В пределе нули заполняют всю лемнискату, т.е. при ее покрытии конечной системой открытых кругов фиксированного малого радиуса $\varepsilon>0$ и при выборе полинома $B_{n}(f, z)$ с достаточно большим номером $n$ получим, что все нули полинома будут находиться внутри покрытия, и в каждый элемент покрытия попадет хотя бы один нуль.

Важное дополнение. Используя этот принцип, надо различать две ситуации.

(а) Выбираются полиномы $B_{n}(f, z)$ с номерами $n=q m$ или $n=q m+1$ при $m \in \mathbb{N}$, т.е. полиномы из цепочки склеиваний (3.8). Тогда все нули подобных полиномов при возрастании номера $n$ стягиваются к лемнискате $\Lambda_{p, q}$.

(b) Выбираются полиномы $B_{n}(f, z)$ с номерами, отличными от указанных в п. (a), т.е. полиномы, не попадающие в цепочку склеиваний (3.8). Тогда при достаточно больших значениях $n$, зависящих от выбора функции (1.2), возможно появление единичных девиантных нулей, имеющих свои предельные точки вне лемнискаты Канторовича $\Lambda_{p, q}$.

Типичные иллюстрации для примера $f(x)=|5 x-2|$ представлены на рис. $2-5$.

Дадим неформальный комментарий к сформулированному выше принципу концентрации нулей. При достаточно большом номере $n \in \mathbb{N}$ полином $B_{n}(f, z)$ имеет высокую степень и скорее всего имеет достаточно много различных нулей. Где расположены эти нули? На основном отрезке $[0,1]$ их быть не может, поскольку полиномы Бернштейна сходятся к своей предельной функции $f(x)=|q x-p|$ монотонно сверху (см. (4.6)). На компакте Канторовича $K_{p, q}$ при больших номерах $n$ нулей также заведомо нет, так как равномерный предел полиномов - функция $\varphi(z)$ из формулы (8.6) - имеет единственный нуль в рассмотренной уже точке $z=p / q \in[0,1]$.

Разложение Поповичу (3.1) показывает, что полином $B_{n}(f, z)$ при любом номере $n \geqslant q$ составлен из структурированных сумм вида (3.6). Вне компакта $K_{p, q}$ слагаемые в таких суммах экспоненциально стремятся к бесконечности (что видно, например, из сравнения формул (5.8) и (8.12)). Соответственно, вдали от $K_{p, q}$ значения полинома $B_{n}(f, z)$ должны быстро расти, и нули просто вынуждены подтягиваться к границе - лемнискате Канторовича $\Lambda_{p, q}$. 


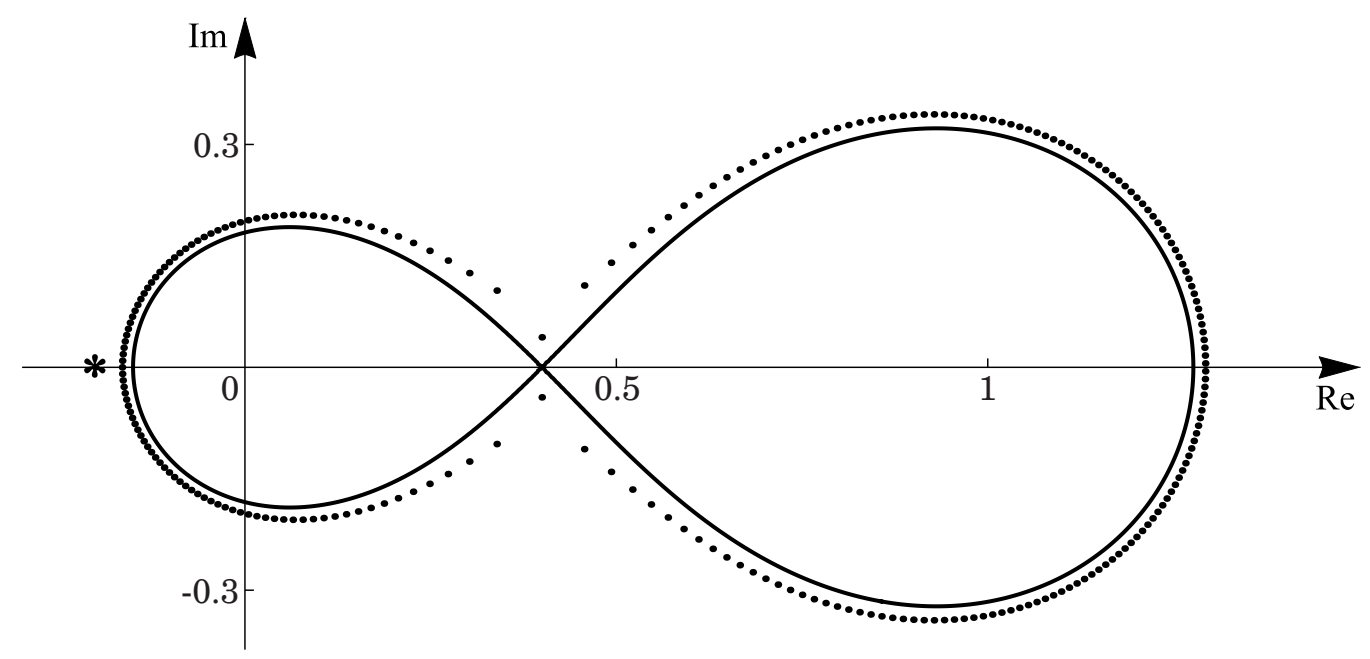

Рис. 3. Множество нулей полинома $B_{252}(f, z)$ для функции $f(x)=|5 x-2|$ вместе с соответствующим аттрактором $\Lambda_{2,5}$. Звездочкой отмечен девиантный нуль $z^{*} \approx-0,216$, возникший из-за того, что полином с номером $n=252$ не попадает в цепочку склеиваний. Компьютерные расчеты показывают, что подобные нули, близкие к $-0,2$, появляются, начиная с номера $n=87$, и далее на всех номерах $n=5 m+2$ при $m \geqslant 18$.

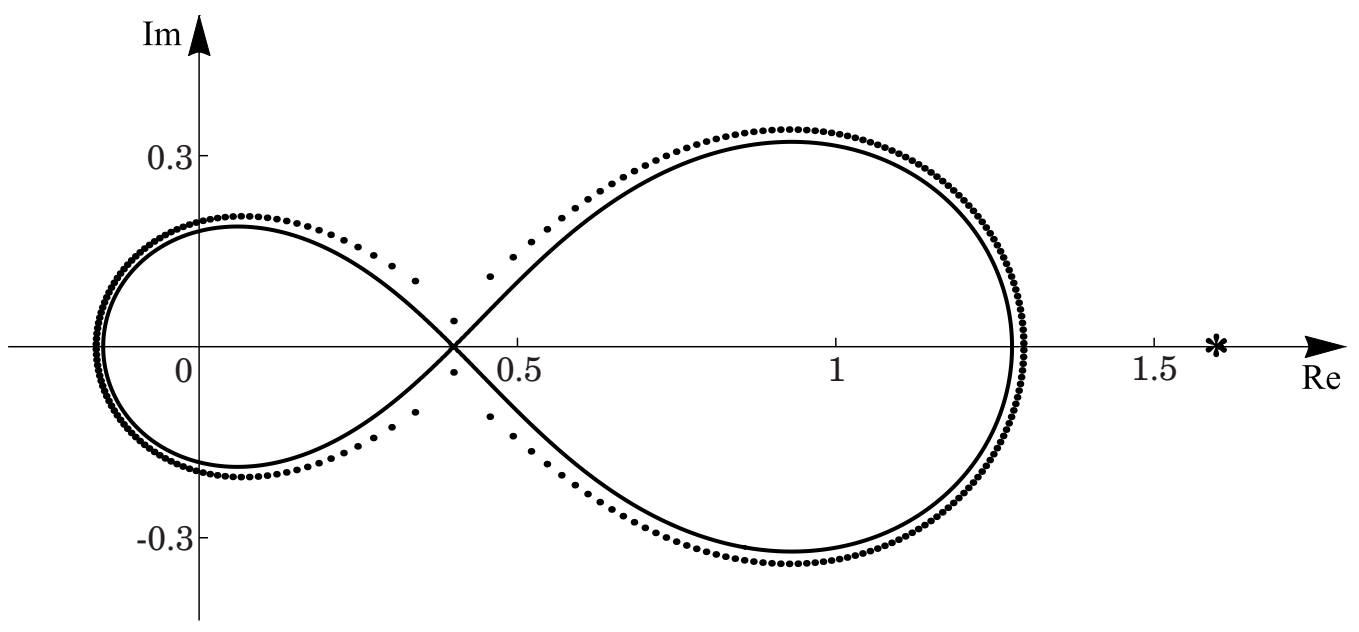

Рис. 4. Множество нулей полинома $B_{253}(f, z)$ для функции $f(x)=|5 x-2|$ вместе с соответствующим аттрактором $\Lambda_{2,5}$. Звездочкой отмечен девиантный нуль $z^{*} \approx 1,6032$, возникший из-за того, что полином с номером $n=253$ не попадает в цепочку склеиваний. Компьютерные расчеты показывают, что подобные нули, близкие к 1,6 , появляются на номерах $n=5 m+3$ при $m \geqslant 3$, т.е. начиная с $n=18$.

В случае, когда полином $B_{n}(f, z)$ попадает в цепочку склеиваний (3.8), структурированные суммы имеют одинаковое число слагаемых, конструкция (3.9) оказывается полностью «сбалансированной», и описанный выше механизм действует безотказно.

Для полиномов, не входящих в цепочку склеиваний, баланс в разложении Поповичу слегка нарушается, и возникающая комбинация (3.10) допускает отдельные девиантные нули на строгом удалении от лемнискаты $\Lambda_{p, q}$. 


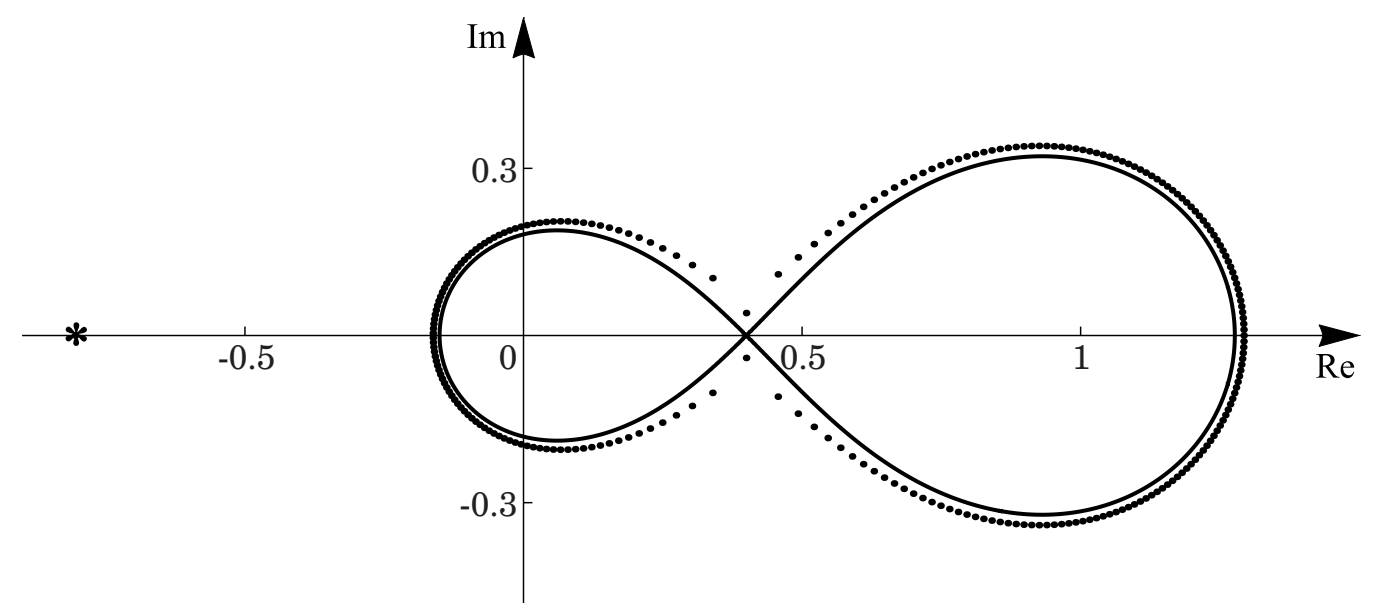

Рис. 5. Множество нулей полинома $B_{254}(f, z)$ для функции $f(x)=|5 x-2|$ вместе с соответствующим аттрактором $\Lambda_{2,5}$. Звездочкой отмечен девиантный нуль $z^{*} \approx-0,8048$, возникший из-за того, что полином с номером $n=254$ не попадает в цепочку склеиваний. Компьютерные расчеты показывают, что подобные нули, близкие к $-0,8$, появляются на номерах $n=5 m+4$ при $m \geqslant 1$, т.е. начиная с $n=9$.

Разумеется, данный неформальный комментарий нельзя считать законченным математическим обоснованием. Это лишь контуры возможной программы будущих исследований. Для удобства дальнейшей работы полезно четко обозначить некоторые цели и выделить ряд конкретных задач, представляющих, на наш взгляд, вполне самостоятельный интерес.

Снова считаем, что $f(x)$ есть фиксированный рациональный модуль вида (1.2), взятый при соглашениях (2.1). Лемниската $\Lambda_{p, q}$ и компакт $K_{p, q}$ определены формулами $(8.3),(8.4)$ соответственно. Сформулируем вопросы, требующие аналитического изучения.

B1. Показать, что при любом фиксированном номере $n \in \mathbb{N}$ все нули полинома $B_{n}(f, z)$ находятся строго вне компакта Канторовича $K_{p, q}$.

B2. Возможны ли кратные нули у полиномов $B_{n}(f, z)$ при каких-либо номерах $n$ ? Как мы увидим ниже (см. п. 14.4), у полиномов, попадающих в цепочку склеиваний (3.8), все нули являются простыми.

В3. При каких номерах $n \in \mathbb{N}$ у полиномов $B_{n}(f, z)$ возможны вещественные нули? Желательно указать как сами номера, так и соответствующие количества вещественных нулей.

B4. Показать, что при $n \rightarrow \infty$ все нули полиномов $B_{n}(f, z)$, попадающих в цепочку склеиваний (3.8), равномерно стягиваются к лемнискате $\Lambda_{p, q}$, заполняя в пределе всю лемнискату.

B5. По-прежнему находясь на цепочке склеиваний, оценить скорость сближения отдельных «экстремальных» нулей с аттрактором - лемнискатой Канторовича $\Lambda_{p, q}$.

B6. Желательно распространить результаты двух предыдущих пунктов на полиномы, не входящие в цепочку склеиваний. При этом придется учитывать возможность появления отдельных девиантных нулей, удаленных от лемнискаты $\Lambda_{p, q}$.

B7. Указать законы образования девиантных нулей у полиномов, не входящих в цепочку склеиваний (3.8). Компьютерные расчеты показывают, что каждая серия полиномов $B_{q m+r}(f, z)$ при фиксированном значении $r \in\{2, \ldots, q-1\}$, начиная с некоторого номера $n_{0}=q m_{0}+r$, дает собственную последовательность девиантных нулей со своим особым поведением. Типичный пример см. на рис. 6.

Покажем на простом примере, что обобщенные разложения Поповичу (3.1) можно эффективно применять к решению поставленных задач.

Теорема 12.1. Пусть $B_{n}(f, z)$ - полиномы Бернштейна (1.1), взятые от симметричного модуля $f(x)=|2 x-1|$. Тогда все нули полиномов $B_{n}(f, z)$ при любом номере $n \in \mathbb{N}$ расположены строго вне компакта Канторовича $K_{1,2}=\{z \in \mathbb{C}: 4|z(1-z)| \leqslant 1\}$. 


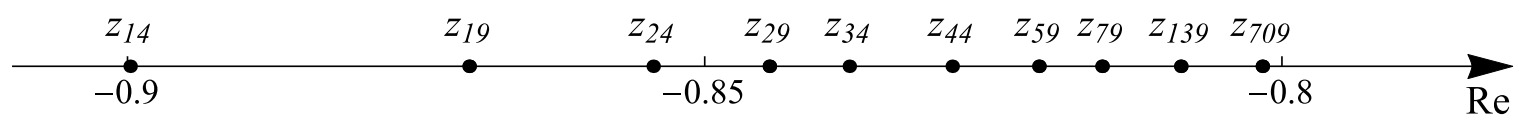

Рис. 6. Компьютерный расчет подпоследовательности девиантных нулей полиномов Бернштейна $B_{n}(f, z)$ для функции $f(x)=|5 x-2|$. Выбраны номера $n=5 m+4$ из множества $n=5 m+4 \in$ $\{14,19,24,29,34,44,59,79,139,709\}$. Нули, скорее всего, сходятся к предельному значению $-0,8=$ $-4 / 5$, находящемуся на некотором удалении от лемнискаты $\Lambda_{2,5}$ (ср. с рис. 5 выше).

Доказательство. В данном случае полином $B_{1}(f, z) \equiv 1$ вообще не имеет нулей. Для прочих полиномов действует классическое разложение (3.13). В силу правила склеивания рассматриваем только полиномы $B_{2 m}(f, z)$ и, согласно (3.13), имеем

$$
B_{2 m}(f, z)=1-\sum_{k=1}^{m} \frac{1}{2 k-1} 2^{-2 k} C_{2 k}^{k}(4 z(1-z))^{k}, \quad m \in \mathbb{N} .
$$

Используя формулу

$$
\frac{1}{2 k-1} C_{2 k}^{k}=4 C_{2 k-2}^{k-1}-C_{2 k}^{k}, \quad k \in \mathbb{N},
$$

при $z=1 / 2$ получаем значение

$$
\begin{aligned}
B_{2 m}(f, 1 / 2)=1-\sum_{k=1}^{m} \frac{1}{2 k-1} 2^{-2 k} C_{2 k}^{k} & =1-\sum_{k=1}^{m} 2^{-2(k-1)} C_{2 k-2}^{k-1}+\sum_{k=1}^{m} 2^{-2 k} C_{2 k}^{k}= \\
& =1-\sum_{k=0}^{m-1} 2^{-2 k} C_{2 k}^{k}+\sum_{k=1}^{m} 2^{-2 k} C_{2 k}^{k}=2^{-2 m} C_{2 m}^{m}, \quad m \in \mathbb{N} .
\end{aligned}
$$

Подробнее про этот известный результат см. [22, с. 153-157].

Пусть теперь $4|z(1-z)| \leqslant 1$. Тогда

$$
\begin{aligned}
\left|B_{2 m}(f, z)\right| \geqslant 1-\sum_{k=1}^{m} \frac{1}{2 k-1} 2^{-2 k} C_{2 k}^{k}(4|z(1-z)|)^{k} \geqslant 1-\sum_{k=1}^{m} \frac{1}{2 k-1} 2^{-2 k} C_{2 k}^{k}= & \\
& =B_{2 m}(f, 1 / 2)=2^{-2 m} C_{2 m}^{m}>0, \quad m \in \mathbb{N} .
\end{aligned}
$$

Тем самым, при всех $m \in \mathbb{N}$ полиномы $B_{2 m}(f, z)\left(=B_{2 m+1}(f, z)\right)$ не имеют нулей на компакте Канторовича $K_{1,2}$. Теорема доказана.

Наглядную иллюстрацию к теореме 12.1 дает рис. 7 .

Попытка перенести рассуждение теоремы 12.1 на общий случай рационального модуля (1.2) потребует, конечно, отдельных усилий. Не исключено также, что часть ответов на поставленные вопросы B1-B7 проще получать, не прибегая к обобщенным разложениям Поповичу (3.1).

13. Особая роль цепочки склеиваний. По-прежнему рассматриваем полиномы Бернштейна (1.1) от рационального модуля (1.2) при соглашениях (2.1). Сохраним специальные обозначения $a_{p, q}(\nu), j_{p, q}(\nu), \varepsilon_{m}(\nu, r)$, введенные при формулировке основной теоремы 3.1 в разделе 3 настоящей работы.

Отмеченные выше примеры с нулями, как и многие другие соображения, показывают, что полиномы, взятые из цепочки склеиваний (3.8), по своим свойствам явно выделяются из общей последовательности полиномов $B_{n}(f, z)$. При номерах $n=q m$, ввиду удачного сочетания абсциссы точки излома $x=p / q$ с точками равномерной сетки (2.3), различные формулы, связанные с полиномами Бернштейна, как правило, существенно упрощаются. 


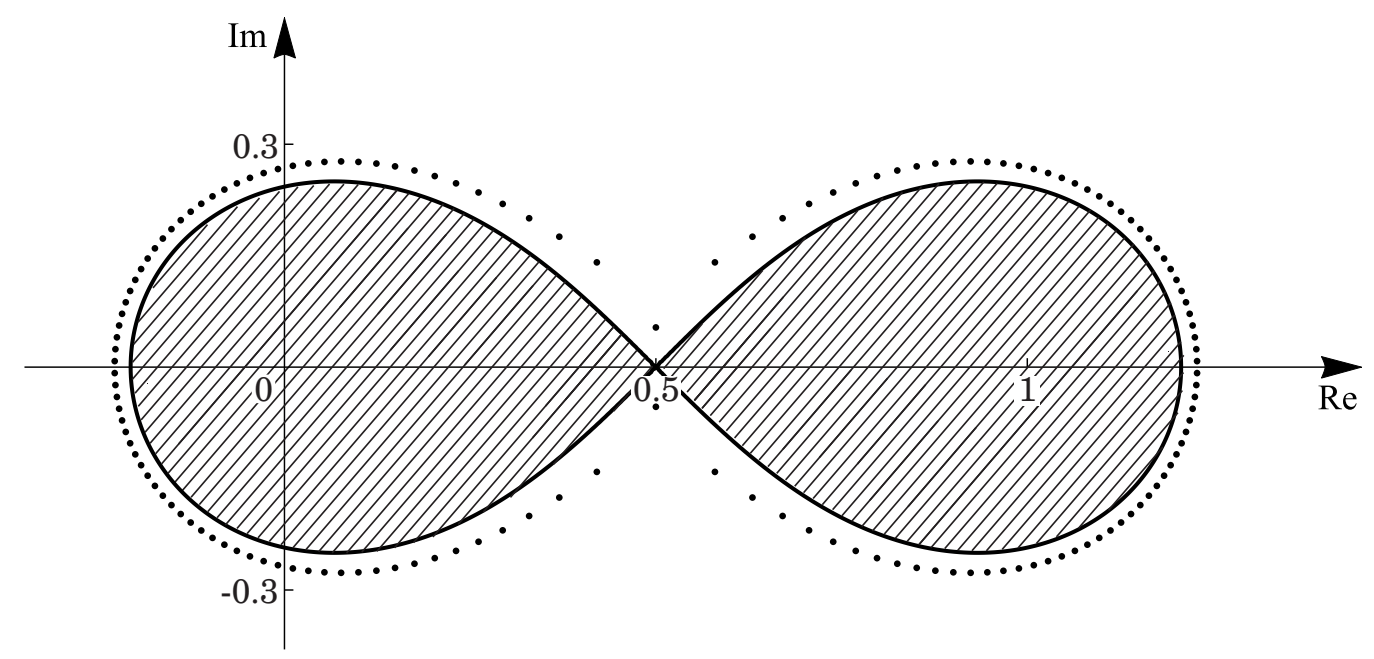

Рис. 7. Множество нулей полинома $B_{150}(f, z)$ для функции $f(x)=|2 x-1|$ вместе с компактом Канторовича $K_{1,2}$. Компакт отмечен штриховкой. Все нули располагаются вне компакта.

В частности, изучаемые нами обобщенные разложения Поповичу $(3.1)$ для полиномов $B_{q m}(f, z)$ при всех $m \in \mathbb{N}$ приобретают полностью сбалансированный вид:

$$
B_{q m}(f, z)=p+(q-2 p) z-\sum_{\nu=1}^{q-1} 2 z^{\lceil p \nu / q\rceil}(1-z)^{\nu-\lfloor p \nu / q\rfloor} \sum_{k=0}^{m-1} \frac{a_{p, q}(\nu)}{q k+\nu} C_{q k+\nu}^{p k+j_{p, q}(\nu)}\left(z^{p}(1-z)^{q-p}\right)^{k}
$$

с одинаковым числом слагаемых во всех структурированных суммах (3.6) (ср. также с прежней формулой (3.9)). По причинам комбинаторного характера полином $B_{q m+1}(f, z)$ совпадает с полиномом $B_{q m}(f, z)$ и может фактически не рассматриваться.

Если функция $f(x)=|q x-p|$ отлична от симметричного модуля $|2 x-1|$, то $q \geqslant 3$, и возникают дополнительные полиномы

$$
B_{q m+r}(f, z), \quad m \in \mathbb{N}, \quad r=2, \ldots, q-1,
$$

которые можно получать из полиномов (13.1), применяя рекуррентное правило (6.2). Запишем в явном виде

$$
\begin{aligned}
B_{q m+r}(f, z) & =B_{q m}(f, z)-\sum_{\nu=1}^{r-1} \frac{2 a_{p, q}(\nu)}{q m+\nu} C_{q m+\nu}^{p m+j_{p, q}(\nu)} z^{p m+\lceil p \nu / q\rceil}(1-z)^{(q-p) m+\nu-\lfloor p \nu / q\rfloor}= \\
& =B_{q m}(f, z)-\left(\sum_{\nu=1}^{r-1} \frac{2 a_{p, q}(\nu)}{q m+\nu} C_{q m+\nu}^{p m+j_{p, q}(\nu)} z^{\lceil p \nu / q\rceil}(1-z)^{\nu-\lfloor p \nu / q\rfloor}\right)\left(z^{p}(1-z)^{q-p}\right)^{m}
\end{aligned}
$$

при $m \in \mathbb{N}$ и $r=2, \ldots, q-1$. Тем самым, если имеются какие-либо алгебраические или комбинаторные соотношения для полиномов $B_{q m}(f, z)$, то они переносятся на полиномы $B_{q m+r}(f, z)$ с учетом конечного числа поправок из (13.2). При этом вид формул может, конечно, заметно «испортиться».

Работая над статьей, авторы обнаружили много соотношений для полиномов $B_{q m}(f, z)$. Часть материала напрямую связана с затронутыми темами, часть - представляет самостоятельный интерес и может указать направление новых исследований. Краткая сводка подобных результатов представлена в следующем разделе.

14. Различные соотношения на цепочке склеиваний. Итак, рассматриваем полиномы Бернштейна (1.1), взятые от рационального модуля (1.2) при соглашениях (2.1). Пусть $n=q m$, 
где $m \in \mathbb{N}$. В таком случае по определению (1.1) имеем

$$
B_{q m}(f, z)=\sum_{k=0}^{q m}\left|\frac{k}{m}-p\right| C_{q m}^{k} z^{k}(1-z)^{q m-k}, \quad m \in \mathbb{N} .
$$

Все последующие результаты в данном разделе относятся именно к этим полиномам.

14.1. Специальные представления изучаемых полиномов. Раскроем модуль в (14.1), разбив общую сумму на две части. Проводя элементарные преобразования и учитывая, что полиномы Бернштейна от линейной функции совпадают с ней самой, получим представление

$$
B_{q m}(f, z)=p-q z+2 \sum_{k=p m+1}^{q m}\left(\frac{k}{m}-p\right) C_{q m}^{k} z^{k}(1-z)^{q m-k}, \quad m \in \mathbb{N} .
$$

Для симметричного модуля $f(x)=|2 x-1|$ подобная формула отмечалась в [22, с. 148].

Помимо (14.2) возможно альтернативное представление

$$
B_{q m}(f, z)=q z-p+2 \sum_{k=0}^{p m-1}\left(p-\frac{k}{m}\right) C_{q m}^{k} z^{k}(1-z)^{q m-k}, \quad m \in \mathbb{N} .
$$

Формулы (14.2) и (14.3) дают явную связь полиномов (14.1) с линейными частями предельной функции $\varphi(z)$, определенной по правилу (8.6).

14.2. Представление первой производной. Известно (см. [36, с. 87] или [49, с. 12]), что первая производная полиномов Бернштейна (1.1) для любой функции $f \in C[0,1]$ допускает запись

$$
B_{n}^{\prime}(f, z)=n \sum_{k=0}^{n-1}\left[f\left(\frac{k+1}{n}\right)-f\left(\frac{k}{n}\right)\right] C_{n-1}^{k} z^{k}(1-z)^{n-1-k}, \quad n \in \mathbb{N} .
$$

Применяя подобное правило к рациональному модулю (1.2), получим для полиномов (14.1) следующее выражение производной:

$$
B_{q m}^{\prime}(f, z)=-q \sum_{k=0}^{p m-1} C_{q m-1}^{k} z^{k}(1-z)^{q m-1-k}+q \sum_{k=p m}^{q m-1} C_{q m-1}^{k} z^{k}(1-z)^{q m-1-k}, \quad m \in \mathbb{N} .
$$

Последнее легко сводится к виду

$$
B_{q m}^{\prime}(f, z)=q-2 q \sum_{k=0}^{p m-1} C_{q m-1}^{k} z^{k}(1-z)^{q m-1-k}, \quad m \in \mathbb{N} .
$$

Из (14.4) следуют граничные соотношения

$$
B_{q m}^{\prime}(f, 0)=-q, \quad B_{q m}^{\prime}(f, 1)=q, \quad m \in \mathbb{N} .
$$

Другими словами, при каждом $m \in \mathbb{N}$ производная $B_{q m}^{\prime}(f, z)$ на концах отрезка $[0,1]$ совпадает с производной $f^{\prime}(x)$ порождающей функции $f(x)=|q x-p|$.

14.3. Связь полинома с его первой производной. Учитывая явное сходство, рассмотрим специальную комбинацию представлений (14.3) и (14.4). Имеем

$$
q B_{q m}(f, z)+(p-q z) B_{q m}^{\prime}(f, z)=2 q \mathfrak{W}_{p, q}^{m}(z),
$$

где

$$
\mathfrak{W}_{p, q}^{m}(z) \equiv \sum_{k=0}^{p m-1}\left(p-\frac{k}{m}\right) C_{q m}^{k} z^{k}(1-z)^{q m-k}+(q z-p) \sum_{k=0}^{p m-1} C_{q m-1}^{k} z^{k}(1-z)^{q m-1-k} .
$$

Используем элементарные соотношения

$$
\left(p-\frac{k}{m}\right) C_{q m}^{k}=p C_{q m-1}^{k}-(q-p) C_{q m-1}^{k-1}, \quad k=1, \ldots, q m-1, \quad C_{q m}^{0}=C_{q m-1}^{0},
$$


и получим, что

$$
\begin{aligned}
\mathfrak{W}_{p, q}^{m}(z)=p \sum_{k=0}^{p m-1} C_{q m-1}^{k} z^{k}(1-z)^{q m-k}-(q-p) \sum_{k=1}^{p m-1} C_{q m-1}^{k-1} z^{k}(1-z)^{q m-k}+ & \\
& +q \sum_{k=0}^{p m-1} C_{q m-1}^{k} z^{k+1}(1-z)^{q m-1-k}-p \sum_{k=0}^{p m-1} C_{q m-1}^{k} z^{k}(1-z)^{q m-1-k}
\end{aligned}
$$

Комбинируя первое слагаемое с четвертым, а затем - с третьим и изменяя порядок суммирования во втором, придем к выражению

$$
\begin{aligned}
& \mathfrak{W}_{p, q}^{m}(z)=(q-p)\left(\sum_{k=0}^{p m-1} C_{q m-1}^{k} z^{k+1}(1-z)^{q m-1-k}-\sum_{k=0}^{p m-2} C_{q m-1}^{k} z^{k+1}(1-z)^{q m-1-k}\right)= \\
&=(q-p) C_{q m-1}^{p m-1} z^{p m}(1-z)^{(q-p) m}
\end{aligned}
$$

Остается подставить результат в исходную формулу и заметить, что $q C_{q m-1}^{p m-1}=p C_{q m}^{p m}$. В итоге приходим к важному тождеству

$$
q B_{q m}(f, z)+(p-q z) B_{q m}^{\prime}(f, z)=2 p(q-p) C_{q m}^{p m}\left(z^{p}(1-z)^{q-p}\right)^{m}, \quad m \in \mathbb{N},
$$

справедливому для полиномов (14.1). Наличие нетривиальной связи (14.6) существенно упрощает многие последующие выводы.

14.4. Простота нулей. Соотношение (14.6) показывает, в частности, что при любом $m \in \mathbb{N}$ все нули полинома $B_{q m}(f, z)$ заведомо являются простыми. Действительно, допустим, что некий полином $B_{q m}(f, z)$ имеет кратный нуль $z_{0} \in \mathbb{C}$, очевидно отличный от $z=0$ и $z=1$. Тогда

$$
B_{q m}\left(f, z_{0}\right)=B_{q m}^{\prime}\left(f, z_{0}\right)=0 .
$$

Подставив точку $z_{0}$ в формулу (14.6), получим противоречие. Результат данного пункта содержит частичный ответ на вопрос В2, поставленный в разделе 12 выше.

14.5. Значение полинома в точке излома. При $z=p / q$ тождество (14.6) сводится к виду

$$
B_{q m}\left(f, \frac{p}{q}\right)=\frac{2 p(q-p)}{q}\left(\frac{p^{p}(q-p)^{q-p}}{q^{q}}\right)^{m} C_{q m}^{p m}, \quad m \in \mathbb{N} .
$$

Итак, при всех $m \in \mathbb{N}$ значения $B_{q m}(f, p / q)$ вычисляются в форме $(14.7)$ для любого рационального модуля $f(x)=|q x-p|$, взятого при соглашениях (2.1). Любопытно, что множитель $2 p(q-p) / q$ совпадает со значением полинома $B_{1}(f, z)=p+(q-2 p) z$ в той же точке излома $z=p / q$.

14.6. Комбинаторные тождества. Пользуясь формулой (14.7), можно установить много различных комбинаторных соотношений. Например, подставим в разложение Поповичу (13.1) значение $z=p / q$ и сравним результат с выражением (14.7). После простых преобразований возникнет неочевидное тождество

$$
\begin{aligned}
& \sum_{\nu=1}^{q-1} \frac{p^{\lceil p \nu / q\rceil-1}(q-p)^{\nu-\lfloor p \nu / q\rfloor-1}}{q^{\nu}} \sum_{k=0}^{m-1} \frac{a_{p, q}(\nu)}{q k+\nu}\left(\frac{p^{p}(q-p)^{q-p}}{q^{q}}\right)^{k} C_{q k+\nu}^{p k+j_{p, q}(\nu)}= \\
&=1-\left(\frac{p^{p}(q-p)^{q-p}}{q^{q}}\right)^{m} C_{q m}^{p m}, \quad m \in \mathbb{N},
\end{aligned}
$$

с величинами $a_{p, q}(\nu), j_{p, q}(\nu)$, вычисляемыми по правилу (3.3). Тождество (14.8) верно при любых значениях $p$ и $q$, удовлетворяющих условиям (2.1). В частности, при $p=1$ с учетом «заготовок», сделанных в примере 7.3, можно свести (14.8) к виду

$$
\sum_{\nu=1}^{q-1} \frac{(q-1)^{\nu-1}}{q^{\nu}} \sum_{k=0}^{m-1} \frac{\nu}{q k+\nu}\left(\frac{(q-1)^{q-1}}{q^{q}}\right)^{k} C_{q k+\nu}^{k}=1-\left(\frac{(q-1)^{q-1}}{q^{q}}\right)^{m} C_{q m}^{m}, \quad m \in \mathbb{N} .
$$


Взяв для конкретики значение $q=3$, получим из (14.9) следующее тождество:

$$
\sum_{k=0}^{m-1} \frac{3}{3 k+1}\left(\frac{4}{27}\right)^{k} C_{3 k+1}^{k}+\sum_{k=0}^{m-1} \frac{4}{3 k+2}\left(\frac{4}{27}\right)^{k} C_{3 k+2}^{k}=9\left(1-\left(\frac{4}{27}\right)^{m} C_{3 m}^{m}\right), \quad m \in \mathbb{N} .
$$

Если же $q=2$, как для симметричного модуля $f(x)=|2 x-1|$, то формула (14.9) путем элементарных преобразований (с учетом равенства $2 C_{2 k-1}^{k-1}=C_{2 k}^{k}$ ) дает соотношение

$$
\sum_{k=1}^{m} \frac{1}{2 k-1} 2^{-2 k} C_{2 k}^{k}=1-2^{-2 m} C_{2 m}^{m}, \quad m \in \mathbb{N} .
$$

По поводу последнего см. также доказательство теоремы 12.1 выше.

Приведем результат иного характера. Вычислим значение $B_{q m}(f, p / q)$ по определению (14.1) и приравняем полученное к правой части в (14.7). Окончательный ответ запишем в виде

$$
\sum_{k=0}^{q m}\left|\frac{k}{m}-p\right| C_{q m}^{k} p^{k}(q-p)^{q m-k}=\frac{2 p(q-p)}{q}\left(p^{p}(q-p)^{q-p}\right)^{m} C_{q m}^{p m}, \quad m \in \mathbb{N} .
$$

Тождество (14.10) имеет прямое отношение к известным биномиальным суммам МуавраПуанкаре (см. специальную статью [39], а также [22, с. 166]).

14.7. Оценка и асимптотика в точке излома. Вновь рассматриваем значения $B_{q m}(f, p / q)$, выраженные явной формулой (14.7). Учитывая стандартную асимптотику (9.6) и двустороннюю оценку (9.8), имеем соотношения

$$
\begin{gathered}
B_{q m}\left(f, \frac{p}{q}\right) \sim \sqrt{\frac{2 p(q-p)}{q m \pi}}, \quad m \rightarrow \infty, \\
\left(1-\frac{q^{2}-p q+p^{2}}{12 p q(q-p) m}\right) \sqrt{\frac{2 p(q-p)}{q m \pi}}<B_{q m}\left(f, \frac{p}{q}\right)<\sqrt{\frac{2 p(q-p)}{q m \pi}}, \quad m \in \mathbb{N} .
\end{gathered}
$$

Из (14.12) следует, что

$$
B_{q m}\left(f, \frac{p}{q}\right)=\sqrt{\frac{2 p(q-p)}{q m \pi}}-\theta_{m}, \quad m \in \mathbb{N},
$$

где бесконечно малая величина $\theta_{m}=\theta_{m}(p, q)>0$ имеет порядок $O\left(m^{-3 / 2}\right)$ при $m \rightarrow \infty$. Формулы (14.11)-(14.13) тесно связаны с результатами Боянича и Чэна [35], полученными сложным аналитическим методом без знания истинного значения (14.7).

Заметим также, что

$$
R_{q m}\left(f, \frac{p}{q}\right)=B_{q m}\left(f, \frac{p}{q}\right)=\frac{2 p(q-p)}{q}\left(\frac{p^{p}(q-p)^{q-p}}{q^{q}}\right)^{m} C_{q m}^{p m}, \quad m \in \mathbb{N},
$$

для уклонения $R_{n}(f, z)$, определенного формулой (8.14). Тем самым, соотношения (14.11)-(14.13) дают возможность уточнить прежнюю оценку (8.16) применительно к точке излома $z=p / q$. Покажем, что величины (14.14) в определенном смысле являются «максимальными» и мажорируют все остальные значения $R_{q m}(f, z)$ на компакте Канторовича $K_{p, q}$.

14.8. Уточненная оценка уклонения. Итак, определим компакт $K_{p, q}$ по формуле (8.4) и рассмотрим уклонения

$$
R_{q m}(f, z) \equiv B_{q m}(f, z)-\varphi(z), \quad z \in K_{p, q}, \quad m \in \mathbb{N}
$$


полиномов (14.1) от предельной функции (8.6). Используя специальные представления (14.2), (14.3), запишем отдельно на левой и правой петлях компакта

$$
\begin{aligned}
& R_{q m}(f, z)=2 \sum_{k=p m+1}^{q m}\left(\frac{k}{m}-p\right) C_{q m}^{k} z^{k}(1-z)^{q m-k}, \quad z \in K_{p, q}^{(1)}, \quad m \in \mathbb{N}, \\
& R_{q m}(f, z)=2 \sum_{k=0}^{p m-1}\left(p-\frac{k}{m}\right) C_{q m}^{k} z^{k}(1-z)^{q m-k}, \quad z \in K_{p, q}^{(2)}, \quad m \in \mathbb{N} .
\end{aligned}
$$

Преобразуем эти выражения к виду

$$
\begin{aligned}
& R_{q m}(f, z)=2\left(z^{p}(1-z)^{q-p}\right)^{m} \sum_{k=p m+1}^{q m}\left(\frac{k}{m}-p\right) C_{q m}^{k}\left(\frac{z}{1-z}\right)^{k-p m}, \quad z \in K_{p, q}^{(1)}, \quad m \in \mathbb{N}, \\
& R_{q m}(f, z)=2\left(z^{p}(1-z)^{q-p}\right)^{m} \sum_{k=0}^{p m-1}\left(p-\frac{k}{m}\right) C_{q m}^{k}\left(\frac{1-z}{z}\right)^{p m-k}, \quad z \in K_{p, q}^{(2)}, \quad m \in \mathbb{N} .
\end{aligned}
$$

Временно обозначим $c=p / q$. Учитывая формулу (8.4) компакта $K_{p, q}$ и вид его петель $K_{p, q}^{(1)}, K_{p, q}^{(2)}$ (см. определение (8.5)), несложно показать, что

$$
\max _{z \in K_{p, q}}\left|z^{p}(1-z)^{q-p}\right|=c^{p}(1-c)^{q-p}, \quad \max _{z \in K_{p, q}^{(1)}}\left|\frac{z}{1-z}\right|=\frac{c}{1-c}, \quad \max _{z \in K_{p, q}^{(2)}}\left|\frac{1-z}{z}\right|=\frac{1-c}{c} .
$$

Но тогда

$$
\left|R_{q m}(f, z)\right| \leqslant R_{q m}(f, c)=R_{q m}\left(f, \frac{p}{q}\right), \quad z \in K_{p, q}, \quad m \in \mathbb{N} .
$$

Значения же $R_{q m}(f, p / q)$ вычисляются по формуле (14.14), и для них справедливы весьма точные оценки (14.11)-(14.13). В итоге всюду на компакте $K_{p, q}$ уклонения (14.15) мажорируются следующим образом:

$$
\left|R_{q m}(f, z)\right| \leqslant R_{q m}\left(f, \frac{p}{q}\right)=\frac{2 p(q-p)}{q}\left(\frac{p^{p}(q-p)^{q-p}}{q^{q}}\right)^{m} C_{q m}^{p m}<\sqrt{\frac{2 p(q-p)}{q m \pi}} .
$$

Универсальная оценка (14.16) действует при всех $z \in K_{p, q}$ и всех $m \in \mathbb{N}$. Она дополняет общие результаты теоремы 8.2 , делая завершенной картину сходимости для полиномов $B_{q m}(f, z)$ из цепочки склеиваний (3.8).

14.9. Представление второй производной. Продифференцировав тождество (14.6), придем к компактной записи второй производной

$$
B_{q m}^{\prime \prime}(f, z)=2 p(q-p) m C_{q m}^{p m} z^{p m-1}(1-z)^{(q-p) m-1}, \quad m \in \mathbb{N} .
$$

Напомним, что подобное соотношение для порождающей функции $f(x)=|x|$ на $[-1,1]$ было впервые отмечено в работе Поповичу [53] (см. раздел 1 нашей статьи). Аналоги (14.17) для произвольного рационального модуля на симметричном отрезке $[-1,1]$ см. в [13]. Формула $(14.17)$ дает универсальное представление $B_{q m}^{\prime \prime}(f, z)$, верное для любого рационального модуля $f(x)=|q x-p|$ на стандартном отрезке $[0,1]$.

Другой возможный способ доказательства (14.17) основан на общей формуле

$$
B_{n}^{\prime \prime}(f, z)=n(n-1) \sum_{k=0}^{n-2}\left[f\left(\frac{k+2}{n}\right)-2 f\left(\frac{k+1}{n}\right)+f\left(\frac{k}{n}\right)\right] C_{n-2}^{k} z^{k}(1-z)^{n-2-k},
$$

действующей при $n \geqslant 2$ для произвольной функции $f \in C[0,1]$ (см. [49, с. 12]). При вычислении разностей второго порядка для нашего рационального модуля (1.2) на номерах $n=q m$ в формуле второй производной останется лишь одно ненулевое слагаемое - отвечающее значению $k=p m-1$ и равное в точности $2 q$. Преобразовав результат, получим прежнее выражение (14.17). 
14.10. Сходимость производных. Для того же рационального модуля $f(x)=|q x-p|$ снова введем компакт Канторовича $K_{p, q}$ и полином Канторовича $T_{p, q}(z)$ по формулам (8.4) и $(8.9)$ соответственно. Согласно теореме 8.1 множество сходимости полиномов $B_{q m}(f, z)$ при $m \rightarrow \infty$ совпадает с компактом $K_{p, q}$. Для второй производной перепишем представление (14.17) в виде

$$
B_{q m}^{\prime \prime}(f, z)=2 q^{q}\left(\frac{z}{p}\right)^{p-1}\left(\frac{1-z}{q-p}\right)^{q-p-1} m\left(\frac{q^{q}}{p^{p}(q-p)^{q-p}}\right)^{-m} C_{q m}^{p m}\left(T_{p, q}(z)\right)^{m-1}, \quad m \in \mathbb{N} .
$$

Тогда, принимая во внимание связь (8.10) и асимптотику (9.6), получим, что полиномы $B_{q m}^{\prime \prime}(f, z)$ при $m \rightarrow \infty$ сходятся к тождественному нулю равномерно на любом компакте в int $K_{p, q}$ и расходятся на множестве $\mathbb{C} \backslash \operatorname{int} K_{p, q}$, в том числе - на границе $\partial K_{p, q}=\Lambda_{p, q}$.

Вопрос о точном описании множества сходимости полиномов $B_{q m}^{\prime}(f, z)$ является более тонким. Тождество (14.6), записанное в эквивалентной форме

$$
q B_{q m}(f, z)+(p-q z) B_{q m}^{\prime}(f, z)=2 p(q-p)\left(\frac{q^{q}}{p^{p}(q-p)^{q-p}}\right)^{-m} C_{q m}^{p m}\left(T_{p, q}(z)\right)^{m}, \quad m \in \mathbb{N},
$$

позволяет утверждать, что при $m \rightarrow \infty$ последовательность производных $B_{q m}^{\prime}(f, z)$ сходится во всех точках $z \in K_{p, q} \backslash\{p / q\}$ (равномерно на компактах из этого множества). При этом, учитывая теорему 8.1, получаем, что

$$
B_{q m}^{\prime}(f, z) \rightarrow \psi(z) \equiv\left\{\begin{array}{rl}
-q, & z \in K_{p, q}^{(1)} \backslash\{p / q\}, \\
q, & z \in K_{p, q}^{(2)} \backslash\{p / q\},
\end{array} \quad m \rightarrow \infty,\right.
$$

с множествами $K_{p, q}^{(1)}$ и $K_{p, q}^{(2)}$, заданными в (8.5). Скорее всего, производные $B_{q m}^{\prime}(f, z)$ сходятся к нулю в точке $z=p / q$ и расходятся во всех точках $z \in \mathbb{C} \backslash K_{p, q}$, но это, конечно, нуждается в отдельном обосновании.

14.11. Степени полиномов и количества нулей. Зафиксируем $m \in \mathbb{N}$. Из (14.17) следует, что

$$
\operatorname{deg} B_{q m}^{\prime \prime}(f, z)=q m-2, \quad \operatorname{deg} B_{q m}^{\prime}(f, z)=q m-1, \quad \operatorname{deg} B_{q m}(f, z)=q m .
$$

При этом полином $B_{q m}^{\prime \prime}(f, z)$ имеет в плоскости $\mathbb{C}$ лишь два различных нуля ${ }^{1}$, а именно, нуль $z=0$ кратности $p m-1$ и нуль $z=1$ кратности $(q-p) m-1$. Очевидно, нули полинома $B_{q m}^{\prime}(f, z)$ отличны от $z=0$ и $z=1$ (см. (14.5)), и потому все они являются простыми для $B_{q m}^{\prime}(f, z)$. Простота нулей полинома $B_{q m}(f, z)$ показана выше в п. 14.4. Но тогда из (14.18) заключаем, что полином $B_{q m}^{\prime}(f, z)$ имеет в точности $q m-1$ простых нулей, а полином $B_{q m}(f, z)$ имеет $q m$ простых нулей. Эта тематика тесно связана с вопросами, поставленными в разделе 12.

Отметим еще, что у полинома $B_{q m}^{\prime}(f, z)$ при любом $m \in \mathbb{N}$ ровно один нуль попадает на интервал $(0,1)$ (ср. формулы $(14.5)$ и $(14.17))$. Полином же $B_{q m}(f, z)$ на $[0,1]$ нулей вовсе не имеет, так как $B_{q m}(f, x)>0$ при $x \in[0,1]$ в силу сходимости к предельной функции $f(x)=|q x-p|$ монотонно сверху (см. формулу (4.6)).

14.12. Вторая производная в точке излома. Подставив в (14.17) значение $z=p / q$, получим, что

$$
B_{q m}^{\prime \prime}\left(f, \frac{p}{q}\right)=2 q^{2} m\left(\frac{p^{p}(q-p)^{q-p}}{q^{q}}\right)^{m} C_{q m}^{p m}, \quad m \in \mathbb{N} .
$$

Очевидная связь между (14.7) и (14.19) приводит к равенству

$$
B_{q m}^{\prime \prime}\left(f, \frac{p}{q}\right)=\frac{q^{3}}{p(q-p)} m B_{q m}\left(f, \frac{p}{q}\right), \quad m \in \mathbb{N} .
$$

Возникает естественный вопрос: можно ли в компактной форме, наподобие (14.7) и (14.19), записать значение первой производной $B_{q m}^{\prime}(f, z)$ в той же точке излома $z=p / q$ ? Для симметричного модуля $f(x)=|2 x-1|$ ответ получается тривиально (ибо $B_{2 m}^{\prime}(f, 1 / 2)=0$ при всех $m \in \mathbb{N}$ ), но в общем случае, для $f(x)=|q x-p|$, задача может оказаться неразрешимой.

\footnotetext{
${ }^{1}$ Точнее, не более двух нулей, поскольку при $m=1$ ситуация может вырождаться.
} 
14.13. Вторая производная как $\delta$-образный объект. Примечательно, что полиномы (14.17) на базовом отрезке $[0,1]$ обладают характерными свойствами $\delta$-образной последовательности, сосредоточенной вблизи точки $x=p / q$. Точнее говоря, верны соотношения

$$
\begin{gathered}
B_{q m}^{\prime \prime}\left(f, \frac{p}{q}\right) \sim q^{2} \sqrt{\frac{2 q m}{p(q-p) \pi}} \rightarrow \infty, \quad m \rightarrow \infty, \\
B_{q m}^{\prime \prime}(f, 0)=B_{q m}^{\prime \prime}(f, 1)=0, \quad m \in \mathbb{N}, \quad m \geqslant 2, \\
B_{q m}^{\prime \prime}(f, x) \sim \sqrt{\frac{2 p q(q-p) m}{\pi}} \frac{\left(T_{p, q}(x)\right)^{m}}{x(1-x)} \rightarrow 0, \quad x \in(0,1) \backslash\{p / q\}, \quad m \rightarrow \infty, \\
\int_{0}^{1} B_{q m}^{\prime \prime}(f, x) d x=2 q, \quad m \in \mathbb{N} .
\end{gathered}
$$

Свойство (14.20) выводим из явного выражения (14.19), используя асимптотику (9.6). Краевые значения (14.21) находим из (14.17). Асимптотическая формула (14.22) также извлекается из $(14.17)$ с учетом поведения на $(0,1)$ первичного полинома Канторовича $T_{p, q}(x)$ (см. $\left.(8.10)\right)$. Величину (14.23) вычисляем через соотношения (14.5) (или стандартным применением эйлеровых интегралов).

14.14. Явная алгебраическая записъ. Раскрывая бином в (14.17), приходим к развернутой алгебраической записи второй производной $B_{q m}^{\prime \prime}(f, z)$ по степеням независимой переменной:

$$
B_{q m}^{\prime \prime}(f, z)=2 p(q-p) m C_{q m}^{p m} \sum_{k=0}^{(q-p) m-1}(-1)^{k} C_{(q-p) m-1}^{k} z^{p m+k-1}, \quad m \in \mathbb{N} .
$$

Интегрируя (14.24) и учитывая условие $B_{q m}^{\prime}(f, 0)=-q$ из (14.5), получаем, что

$$
B_{q m}^{\prime}(f, z)=-q+2 p(q-p) m C_{q m}^{p m} \sum_{k=0}^{(q-p) m-1} \frac{(-1)^{k}}{p m+k} C_{(q-p) m-1}^{k} z^{p m+k}, \quad m \in \mathbb{N} .
$$

Наконец, интегрирование (14.25) с учетом условия $B_{q m}(f, 0)=p$ дает при всех $m \in \mathbb{N}$ разложение по степеням $z$ для самих полиномов Бернштейна

$$
B_{q m}(f, z)=p-q z+2 p(q-p) m C_{q m}^{p m} \sum_{k=0}^{(q-p) m-1} \frac{(-1)^{k}}{(p m+k)(p m+k+1)} C_{(q-p) m-1}^{k} z^{p m+k+1} .
$$

Подобная формула для симметричного модуля $f(x)=|2 x-1|$ подробно изучена в [19].

В общем же случае особый интерес представляет задача о скорости роста коэффициентов в полиномах Бернштейна при явной алгебраической записи (14.26). Требуется выяснить, например, зависимость скорости роста максимального коэффициента от выбора значений $p, q$. Как следует из результатов [20], наибольший асимптотический рост максимального коэффициента будет наблюдаться в полиномах Бернштейна для функции $f(x)=|3 x-1|$, т.е. при $p=1$ и $q=3$.

14.15. Пределы структурированных сумм. Используем пример $f(x)=|3 x-1|$ для постановки еще одной интересной проблемы. В этом случае, как показывает формула $(3.14)$, при $x \in[0,1]$ для полиномов $B_{3 m}(f, x)$ из цепочки склеиваний справедливо представление

$$
\begin{aligned}
B_{3 m}(f, x)=1+x-2 x(1-x) \sum_{k=0}^{m-1} & \frac{1}{3 k+1} C_{3 k+1}^{k}\left(x(1-x)^{2}\right)^{k}- \\
& -2 x(1-x)^{2} \sum_{k=0}^{m-1} \frac{2}{3 k+2} C_{3 k+2}^{k}\left(x(1-x)^{2}\right)^{k}, \quad m \in \mathbb{N} .
\end{aligned}
$$


Сходимость полиномов понятна: если $m \rightarrow \infty$, то

$$
B_{3 m}(f, x) \rightrightarrows f(x) \equiv|3 x-1| \quad \text { на множестве } x \in[0,1] .
$$

Напрашивается логичный вопрос: к чему по отдельности сходятся структурированные суммы в формуле (14.27)? Ответ оказывается весьма неожиданным: можно показать, что

$$
\begin{aligned}
2 x(1-x) \sum_{k=0}^{\infty} \frac{1}{3 k+1} C_{3 k+1}^{k}\left(x(1-x)^{2}\right)^{k} & =4 \sqrt{\frac{x}{3}} \sin \left(\frac{1}{3} \arcsin \left(\frac{3}{2}(1-x) \sqrt{3 x}\right)\right), \\
2 x(1-x)^{2} \sum_{k=0}^{\infty} \frac{2}{3 k+2} C_{3 k+2}^{k}\left(x(1-x)^{2}\right)^{k} & =\frac{8}{3} \sin ^{2}\left(\frac{1}{3} \arcsin \left(\frac{3}{2}(1-x) \sqrt{3 x}\right)\right)
\end{aligned}
$$

при $x \in[0,1]$. Совершая теперь предельный переход (14.28), получим неочевидное тождество

$$
|3 x-1|=1+x-4 \sqrt{\frac{x}{3}} \sin \left(\frac{1}{3} \arcsin \left(\frac{3}{2}(1-x) \sqrt{3 x}\right)\right)-\frac{8}{3} \sin ^{2}\left(\frac{1}{3} \arcsin \left(\frac{3}{2}(1-x) \sqrt{3 x}\right)\right),
$$

заведомо верное при $x \in[0,1]$. Любопытно, что

$$
4 \sqrt{\frac{x}{3}} \sin \left(\frac{1}{3} \arcsin \left(\frac{3}{2}(1-x) \sqrt{3 x}\right)\right)=\frac{8}{3} \sin ^{2}\left(\frac{1}{3} \arcsin \left(\frac{3}{2}(1-x) \sqrt{3 x}\right)\right)=2 x
$$

при $x \in[0,1 / 3]$, но эти же тригонометрические выражения становятся нелинейными на промежутке $x \in[1 / 3,1]$. Вывод (14.29) основан на равенстве

$$
\sin \left(\frac{1}{3} \arcsin \left(\frac{3}{2}(1-x) \sqrt{3 x}\right)\right)=\frac{\sqrt{3 x}}{2}, \quad 0 \leqslant x \leqslant \frac{1}{3} .
$$

Последнее легко проверить при помощи формулы синуса тройного угла.

Желательно распространить подобные результаты на случай произвольного рационального модуля (1.2). Авторы располагают полным решением проблемы для $f(x)=|q x-1|$, когда согласно примеру 7.3 полиномы $B_{q m}(f, x)$ структурируются на основе «регулярной» формулы

$$
B_{q m}(f, x)=1+(q-2) x-\sum_{\nu=1}^{q-1} 2 x(1-x)^{\nu} \sum_{k=0}^{m-1} \frac{\nu}{q k+\nu} C_{q k+\nu}^{k}\left(x(1-x)^{q-1}\right)^{k},
$$

более удобной, чем в общем случае $f(x)=|q x-p|$.

Задаче о нахождении раздельных пределов при $m \rightarrow \infty$ для структурированных сумм в разложении (14.30) планируется посвятить специальную работу.

\section{СПИСОК ЛИТЕРАТУРЫ}

1. Бернштейн C. H. Sur la convergence de certaines suites de polynomes// в кн.: Собрание сочинений. - Изд-во АН СССР, 1954. - Т. 2. Конструктивная теория функций [1931-1953]. - С. 187-197; см. также: Bernstein S. Sur la convergence de certaines suites de polynomes// J. Math. — 1936. — 15. — P. $345-358$.

2. Бернштейн С. Н. О сходимости многочленов $\sum_{0}^{n} C_{n}^{m} f(m / n) x^{m}(1-x)^{n-m}$ в комплексной области// Изв. АН СССР. Сер. мат. - 1943. - 7, № 2. - С. 49-88.

3. Виденский В. С. Многочлены Бернштейна. - Л.: ЛГПИ им. А. И. Герцена, 1990.

4. Гончаров В. Л. Теория интерполирования и приближения функций. - М.: ГИТТЛ, 1954.

5. Грэхем Р., Кнут Д., Паташник О. Конкретная математика. Основание информатики. - М.: Мир, 1998.

6. Ильин В. А. О ещё одном выводе формулы Стирлинга// Мат. высш. образ. - 2007. - № 5. - С. 9-14.

7. Канторович Л. В. О сходимости последовательности полиномов С. Н. Бернштейна за пределами основного интервала// Изв. АН СССР. VII сер. - 1931. - № 8. - С. 1103-1115.

8. Коровкин П. П. Линейные операторы и теория приближений. - М.: Физматгиз, 1959. 
9. Маркущевич А. И. Теория аналитических функций. Т. 1. Начала теории. - М.: Наука, 1967.

10. Монтель П. Нормальные семейства аналитических функций. - М.-Л.: ОНТИ НКТП СССР, 1936.

11. Натансон И. П. Конструктивная теория функций. - М.-Л.: ГИТТЛ, 1949.

12. Новиков И. Я. Асимптотика корней полиномов Бернштейна, используемых в построении модифицированных всплесков Добеши// Мат. заметки. - 2002. - 71, № 2. - С. 239-253.

13. Петросова М. А. О некоторых соотношениях, связанных с полиномами Бернштейна для рационального модуля на симметричном отрезке// в кн.: Некоторые актуальные проблемы современной математики и математического образования/ Науч. конф. «Герценовские чтения» (9-13 апреля 2018). СПб.: Изд. РГПУ им. А. И. Герцена, 2018. - С. 153-157.

14. Полиа Г., Сеге Г. Задачи и теоремы из анализа. Т. 1. Ряды. Интегральное исчисление. Теория функций. - М.: Наука, 1978.

15. Полищук В. И. О формуле Стирлинга/ (частное сообщение).

16. Попов А. Ю. Двусторонние оценки сумм значений функции в целых точках и их приложения. Переславль-Залесский, 2016.

17. Попов А. Ю. Оценка сверху остатка степенного ряда с положительными коэффициентами специального вида// Челябинск. физ.-мат. ж. - 2017. - 2, № 2. - С. 193-198.

18. Титчмари Е. Теория функций. - М.: Наука, 1980.

19. Тихонов И. В., Шерстюков В. Б. Приближение модуля полиномами Бернштейна// Вестн. Челябинск. гос. ун-та. Мат. Мех. Информ. - 2012. - 15, № 26. - С. 6-40.

20. Тихонов И. В., Шерстюков В. Б. О поведении коэффициентов полиномов Бернштейна при алгебраической записи на стандартном отрезке// в кн.: Некоторые актуальные проблемы современной математики и математического образования / Науч. конф. «Герценовские чтения» (13-17 апреля 2015). СПб.: Изд. РГПУ им. А. И. Герцена, 2015. - С. 115-121.

21. Тихонов И. В., Шерстюков В. Б. Полный анализ сходимости полиномов Бернштейна от симметричного модуля// Изв. Саратов. ун-та. Нов. сер. Мат. Мех. Информ. - 2020 (в печати).

22. Тихонов И. В., Шерстюков В. Б., Петросова М. А. Полиномы Бернштейна: старое и новое// в кн.: Математический форум/ Исследования по математическому анализу. - Владикавказ: ЮМИ ВНЦ РАН и РСО-А, 2014. - 8. - С. 126-175.

23. Тихонов И. В., Шерстюков В. Б., Петросова М. А. Правило склеивания для полиномов Бернштейна на симметричном отрезке// Изв. Саратов. ун-та. Нов. сер. Мат. Мех. Информ. -2015 . -15 , № 3 . C. $288-300$.

24. Тихонов И. В., Шерстюков В. Б., Петросова М. А. Полиномы Бернштейна для стандартного модуля на симметричном отрезке// Изв. Саратов. ун-та. Нов. сер. Мат. Мех. Информ. -2016 . - 16, № 4 . C. $425-435$.

25. Тихонов И. В., Шерстюков В. Б., Цветкович Д. Г. Специальные задачи для полиномов Бернштейна в комплексной области// в кн.: Некоторые актуальные проблемы современной математики и математического образования/ Науч. конф. «Герценовские чтения-2016» (11-15 апреля 2016). - СПб.: Изд. РГПУ им. А. И. Герцена, 2016. - С. 139-145.

26. Тихонов И. В., Цветкович Д. Г., Шерстюков В. Б. Компьютерное исследование аттракторов нулей для классических полиномов Бернштейна// Фундам. прикл. мат. - 2016. - 21, № 4. - С. 151-173.

27. Тихонов И. В., Шерстюков В. Б., Цветкович Д. Г. Что такое аттракторы нулей для классических полиномов Бернштейна// в кн.: Некоторые актуальные проблемы современной математики и математического образования/ Науч. конф. «Герценовские чтения-2017» (10-14 апреля 2017). - СПб.: Изд. РГПУ им. А. И. Герцена, 2017. - С. 150-160.

28. Тихонов И. В., Шерстюков В. Б., Цветкович Д. Г. Об одном методе для нахождения области сходимости полиномов Бернштейна в комплексной плоскости// в кн.: Некоторые актуальные проблемы современной математики и математического образования/ Науч. конф. «Герценовские чтения-2018» (9-13 апреля 2018). - СПб.: Изд. РГПУ им. А. И. Герцена, 2018. - С. 145-153.

29. Фихтенгольи, Г. М. Основы математического анализа. Т. 2. - М.: Наука, 1968.

30. Цветкович Д. Г., Тихонов И. В., Шерстюков В. Б. Специальные представления для полиномов Бернштейна от рационального модуля на стандартном отрезке// в кн.: Современные проблемы теории функций и их приложения. - Саратов: Научная книга, 2018. - С. 339-342.

31. Цветкович Д. Г. Особые структурированные представления для полиномов Бернштейна от рационального модуля// в кн.: Системы компьютерной математики и их приложения/ XIX Междунар. науч. конф., посв. 100-летию физ.-мат. ф-та СмолГУ. - Смоленск: СмолГУ, 2018. - С. 347-353. 
32. Цветкович Д. Г. Подробный атлас аттракторов нулей для классических полиномов Бернштейна// Челябинск. физ.-мат. ж. - 2018. - 3, № 1. - С. 58-89.

33. Юшкевич А. П. (ред.). История математики с древнейших времен до начала ХІХ столетия. Т. 2. Математика XVII столетия. - М.: Наука, 1970.

34. Aramă O. Proprietăţi privind monotonia şirului polinoamelor de interpolare ale lui S. N. Bernstein şi aplicarea lor la studiul aproximării funcţiilor// Stud. Cercet. Mat. (Cluj). — 1957. — 8, № 3-4. — P. 195210.

35. Bojanich R., Cheng F. Rate of convergence of Bernstein polynomials for functions with derivatives of bounded variation// J. Math. Anal. Appl. — 1989. — 141, № 1. — P. 136-151.

36. Bustamante J. Bernstein Operators and Their Properties. — Birkhäuser, 2017.

37. Davis P. J. Interpolation and Approximation. - N.Y.: Dover, 1975.

38. DeVore R. A., Lorentz G. G. Constructive Approximation. - Berlin-Heidelberg-New York: SpringerVerlag, 1993.

39. Diaconis P., Zabell S. Closed form summation for classical distributions: variations on a theme of de Moivre// Stat. Sci. - 1991. - 6, № 3. - P. 284-302.

40. Dvoretzky A. On the theorem of Jentzsch// Proc. Natl. Acad. Sci. U.S.A. - 1949. — 35. - P. $246-252$.

41. Fowler D. The binomial coefficient function// Am. Math. Month. - 1996. — 103, № 1. — P. 1-17.

42. Gal S. G. Approximation by complex Bernstein and convolution type operators. - London-Singapore: World Scientific, 2009.

43. Gehlen W., Luh W. On the sharpness of Jentzsch-Szegö-type theorems// Arch. Math. — 1994. — 63. P. 33-38.

44. Izumi S. On the distribution of the zero points of sections of a power series// Jpn. J. Math. — 1927. — 4. - P. 29-32.

45. Jentzsch R. Untersuchungen zur Theorie der Folgen analytischer Funktionen// Acta Math. — 1918. - 41. - P. 219-251.

46. Knuth D. E., Vardi I. The asymptotic expansion of the middle binomial coefficient// Am. Math. Month. - 1996. - 97, № 7. - P. 629-630.

47. Kocić Lj. M., Della Veccia B. Degeneracy of positive linear operators// Facta Univ. Ser. Math. Inform. 1998. - 13. - P. 59-72.

48. Landau E., Gaier D. Darstellung und Begründung einiger neuerer Ergebnisse der Funktionentheorie. Berlin-Heidelberg-New York: Springer-Verlag, 1986.

49. Lorentz G. G. Bernstein polynomials. - Toronto: Univ. of Toronto Press, 1953.

50. Passow E. Some unusual Bernstein polynomials// in: Approximation Theory IV (Chui C. K., Schumaker L. L., Ward J. D., eds.)/ Int. Symp. on Approximation Theory (Texas, January 10-14, 1983). - New York-London: Academic Press, 1983. - P. 649-652.

51. Passow E. Deficient Bernstein polynomials// J. Approx. Th. - 1989. — 59, № 3. — P. $282-285$.

52. Phillips G. M. Interpolation and approximation by polynomials. - New York-Berlin-Heidelberg: SpringerVerlag, 2003.

53. Popoviciu T. Sur l'approximation des fonctions convexes d'ordre supérieur// Mathematica. — 1935. - 10. - P. 49-54.

54. Rahman Q. I., Schmeisser G. Analytic Theory of Polynomials. - Oxford: Clarendon Press, 2002.

55. Rodney E. Problem 10310// Am. Math. Month. — 1993. — 100, № 5. - P. 499; см. также: Rodney E. Solution problem 10310// Am. Math. Month. — 1996. — 103, № 5. - P. 431-432.

56. Sasvári Z. Inequalties for binomial coefficients// J. Math. Anal. Appl. — 1999. — 236, № 1. — P. $223-226$.

57. Schoenberg I. J. On variation diminishing approximation methods// in: On Numerical Approximation (Langer R. E., ed.)/ Symp. conducted by the Mathematics Research Center US Army (University of Wisconsin, Madison, April 21-23, 1958). - Madison: Univ. of Wisconsin Press, 1959. — P. 249-274.

58. Stănică P. Good lower and upper bounds on binomial coefficients// J. Inequal. Pure Appl. Math. — 2001. -2 , № 3. - P. 1-5.

59. Temple W. B. Stieltjes integral representation of convex functions// Duke Math. J. — 1954. — 21, № 3. P. 527-531.

60. Vallée Poussin Charles de la On the approximation of functions of a real variable and on quasi-analytic functions// Rice Inst. Pamhlet. - 1925. - 12, № 2. - P. 105-172. 
61. Wright E. M. The Bernstein approximation polynomials in the complex plane// J. London Math. Soc. 1930. - 5, № 4. - P. 265-269.

Тихонов Иван Владимирович

Московский государственный университет им. М. В. Ломоносова

E-mail: ivtikh@mail.ru

Шерстюков Владимир Борисович

Национальный исследовательский ядерный университет «МИФИ», Москва

E-mail: shervb73@gmail.com

Цветкович Диана Горановна

Московский педагогический государственный университет

E-mail: dianacve@inbox.ru 